University of Redlands

\title{
Automating Wetland Prioritization Analyses Using GIS
}

\author{
A Major Individual Project submitted in partial satisfaction of the requirements \\ for the degree of Master of Science in Geographic Information Systems \\ by \\ Blythe Hawley Spendlove \\ Fang Ren, Ph.D., Committee Chair \\ Mark Kumler, Ph.D.
}

July 2018 
Automating Wetland Prioritization Analyses Using GIS

Copyright (C) 2018

by

Blythe Hawley Spendlove 
The report of Blythe Hawley Spendlove is approved.

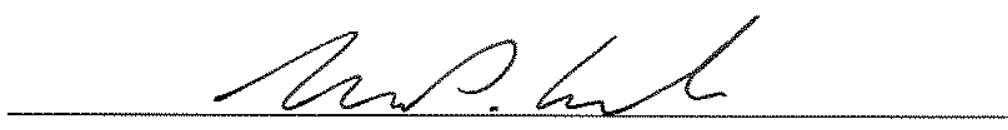

Mark Kumler, Ph.D.

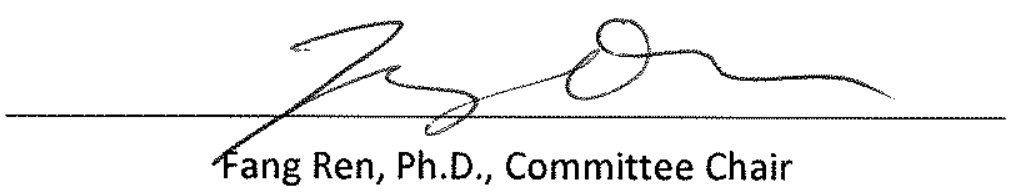

August 2018 



\section{Acknowledgements}

I have so many people who have helped me get to this point in my life. I cannot even begin a list sufficient enough to include them all. I would especially like to thank and acknowledge the help and support of my family, Dr. Fang Ren, the other MSGIS faculty and adjunct faculty at the University of Redlands, and Cohort 32 of the MSGIS program. None of this would have been possible without their support and assistance.

In addition, I would like to thank all the Spatial Studies staff at the University of Redlands for their assistance, including Nathan Strout, Lisa Benvenuti, and Matt Flewelling. I would also like to thank Jonathan Quinn for helping me with my geoprocessing service, the Morrison Foundation Scholarship and the Fred and Blythe Keenan Educational Trust for helping fund my education, Ghirardelli for making chocolate, and God, who made everything possible. 



\section{Abstract \\ Automating Wetland Prioritization Analyses Using GIS}

by

Blythe Hawley Spendlove

In environmental and conservation fields, managers and other decision makers need to prioritize their efforts to specific areas using multiple-criteria decision analysis, to maximize environmental protection given limitations of budget and time. However, creating these prioritization models requires a combination of both scientific and technical skills, and many of those with the expertise to create scientifically sound prioritization models have limited time to devote to the technical aspects of the analysis. There was a need to automate this analysis process to enable scientists and other decision makers to quickly repeat analyses with different criteria and compare the results.

This project automated a wetland prioritization analysis in California for the U.S. Fish and Wildlife Service (USFWS) into a collection of ArcGIS ModelBuilder tools. This project then created a GIS web application to enable USFWS employees to re-do the prioritization analysis with different weights for the various ecological factors that were included in the analysis (such as endangered species habitat, important bird areas, etc.). With the analysis process thus simplified, scientists and decision makers in the USFWS can now apply current and evolving scientific knowledge and compare wetland priorities in a multiple criteria decision analysis framework. 



\section{Table of Contents}

Chapter 1 - Introduction ................................................................................................. 1

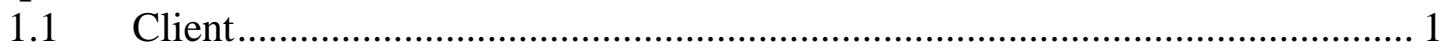

$1.2 \quad$ Problem Statement ................................................................................ 1

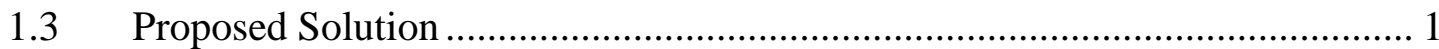

1.3.1 Goals and Objectives .......................................................................... 2

1.3.2 Scope

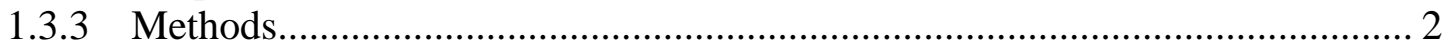

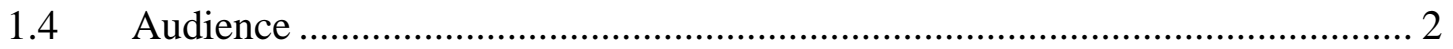

1.5 Overview of the Rest of this Report ............................................................... 3

Chapter 2 - Background and Literature Review .............................................................. 5

2.1 Wetland Prioritization - Previous Efforts and Criterion for Prioritization....... 5

2.1.1 Criterion for Wetland Prioritization .............................................................. 5

2.1.2 Previous Work on Prioritizing Wetlands with GIS …………………………... 6

2.2 Multiple Criteria Decision Analysis and GIS ............................................... 6

2.3 Appropriate Buffer Distance for Wetland Prioritization .................................. 7

2.4 Miscellaneous Note on Vector vs. Raster Overlay Research ........................... 8

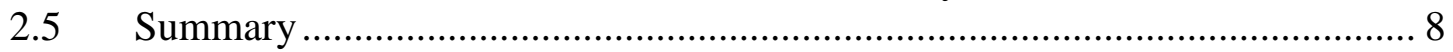

Chapter 3 - Systems Analysis and Design......................................................................... 9

$3.1 \quad$ Problem Statement .......................................................................... 9

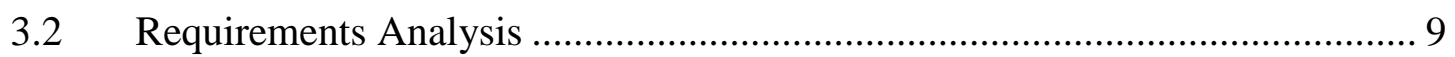

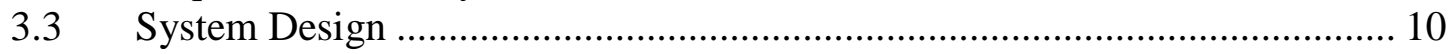

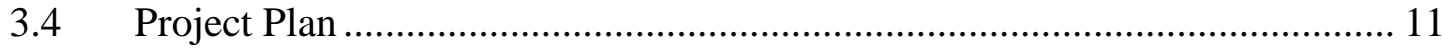

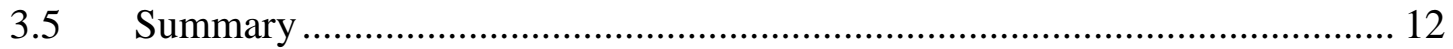

Chapter 4 - Database Design.............................................................................................. 13

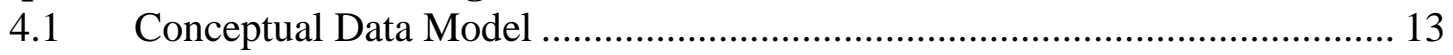

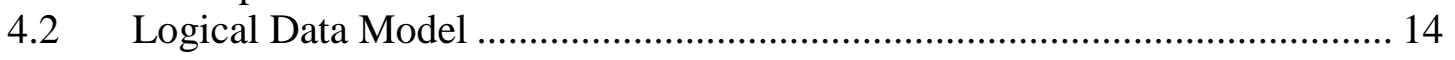

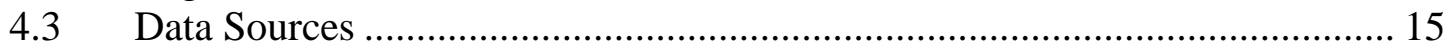

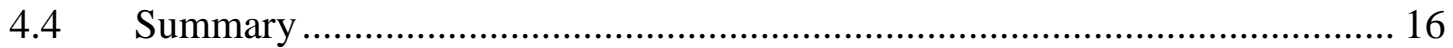

Chapter 5 - Implementation.............................................................................................. 17

5.1 Introduction to Wetland Prioritization Toolbox …………........................... 17

5.2 Important Bird Areas Data Preparation and Automation ................................ 18

5.3 Critical Habitat Data Preparation and Automation ......................................... 19

5.4 Critical Habitat Lines Data Preparation and Automation ................................ 22

$5.5 \quad$ Wetland Data Preparation ......................................................................... 24

5.6 Wetland Prioritization Analysis ..................................................................... 28

5.7 Wetland Prioritization Geoprocessing Service ……………………………..... 31

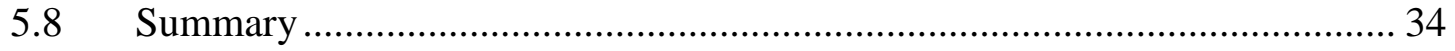

Chapter 6 - Results and Analysis..................................................................................... 35

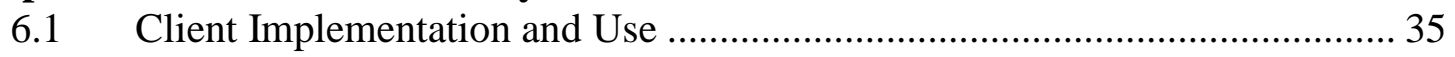

6.2 Benefits of This Project …………………………………………….... 41

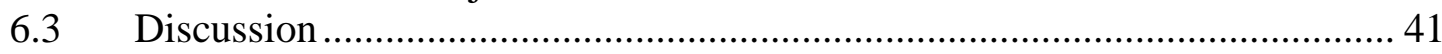




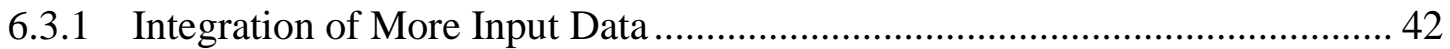

6.3.2 Further Research on Buffer Distance and Area Thresholds ......................... 42

6.3.3 Integration of Fragmentation and Connectivity Models ................................ 48

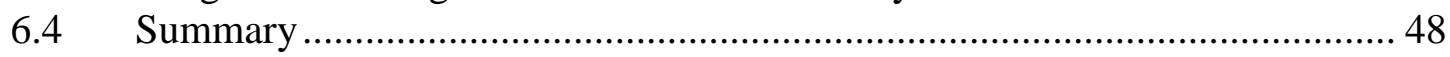

Chapter 7 - Conclusions and Future Work ................................................................. 49

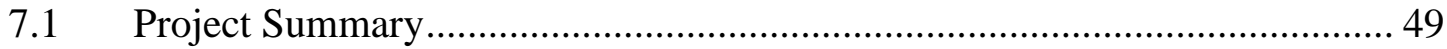

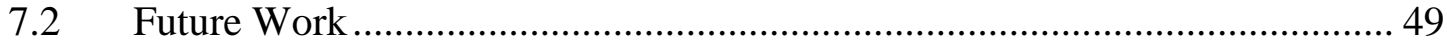

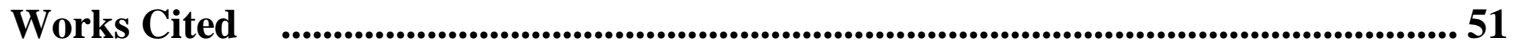

Appendix A. Models, Scripts, and Script Tools Created in the Project .................. 53

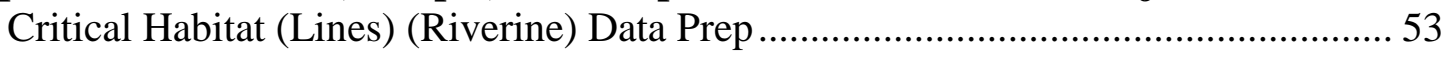

Critical Habitat (Polygons) Data Prep ................................................................. 58

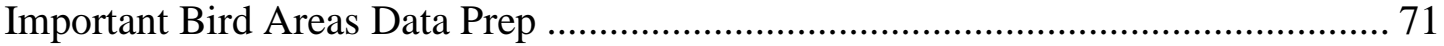

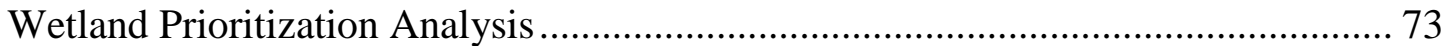

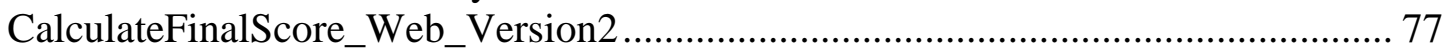

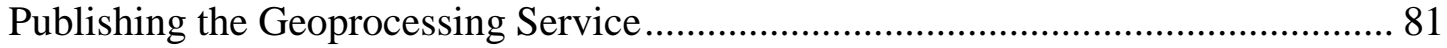




\section{Table of Figures}

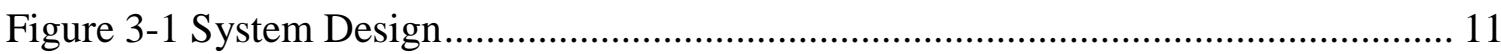

Figure 3-2 Project Plan ..................................................................................... 11

Figure 4-1 - Conceptual Data Model...................................................................... 13

Figure 4-2: Logical Data Model ............................................................................. 14

Figure 5-1: Toolsets, Models, and Script Tools within Wetland Prioritization Toolbox . 17

Figure 5-2: Incorporating Overlapping Critical Habitat Areas for Individual Species .... 21

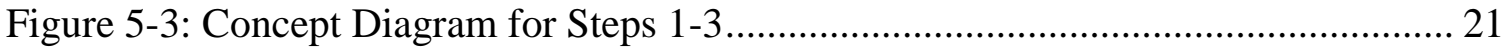

Figure 5-4: Critical Habitat Symbolized by Species Count and Standardized Score ....... 22

Figure 5-5: Count and Standardized Score Comparison for Critical River Habitat ......... 24

Figure 5-6: Only wetlands within 500 meters of prioritization criteria were kept ........... 26

Figure 5-7: Original Wetland Area Compared Against Simplified Outline ...................... 26

Figure 5-8: Aggregated Wetlands compared to Final Wetland Output for Prioritization

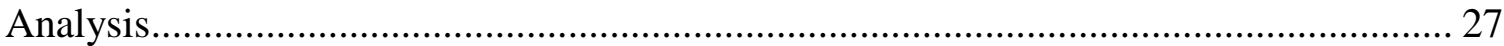

Figure 5-9: San Pablo Bay Area ............................................................................. 28

Figure 5-10: Prioritization Factors were combined into one dataset using "Union"........ 30

Figure 5-12: Calculate Fields Dialog Box in Wetland Prioritization Analysis Step 4 ..... 31

Figure 5-14: Roads and Labels Appear on Top of Prioritized Wetlands for Reference ... 33

Figure 6-3: Map of Wetlands in the San Pablo Bay Area................................................. 37

Figure 6-4: Prioritize Wetlands Pane with Custom Weights Entered.............................. 38

Figure 6-6: Close-up Map of Wetland Prioritized near Fairfield ……………………..... 39

Figure 6-7: Print Button in Web Application ................................................................. 40

Figure 6-9: Wetland Prioritization in a Multi-Criteria Decision Analysis Framework .... 41

Figure 6-10: Example of Aggregated and Non-Aggregated Wetlands ............................ 43

Figure 6-11: South of Fairfield Study Area ..................................................................... 44

Figure 6-12: Statistics for Original Wetland Data in the Study Area ............................... 44

Figure 6-13: Comparison of the Effects of Aggregation Distance Thresholds ................ 45

Figure 6-14: Comparison of the Increase in Wetland Area and Decrease in Vertex Count with Aggregation Distance .................................................................................... 46

Figure 6-15: Change in Estimated Wetland Area with Aggregation Distances of 500, 400, 300 and 200 meters in one area, and 200,150, 100 and 50 meters in a smaller sub-region.

Figure A-1: Toolsets in Wetland Prioritization Toolbox ................................................ 53

FigureA-2: Critical Habitat (Lines) (Riverine) Data Prep toolbox .................................... 53

Figure A-3: CritHabLines_Step1 ............................................................................ 54

Figure A-4: CritHabLines_Step 2 ............................................................................. 55

Figure A-5: Background Process in Step 2

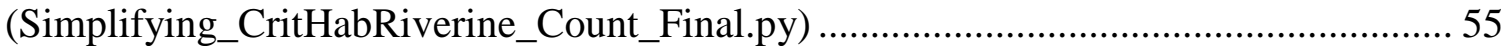

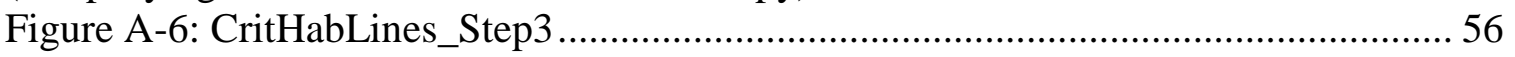

Figure A-7: CritHabLines_Step4_CleanUp................................................................. 56

Figure A-8 Geoprocessing Pane Interface for CritHabLines_Step1 and

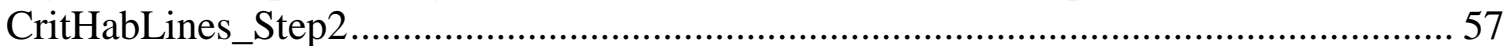

Figure A-9: Geoprocessing Pane Interface for CritHabLines_Step3 and

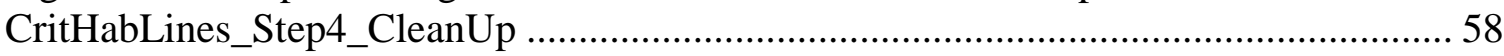


Figure A-10: Critical Habitat Prep Background Process .................................................. 59

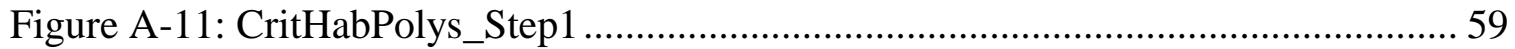

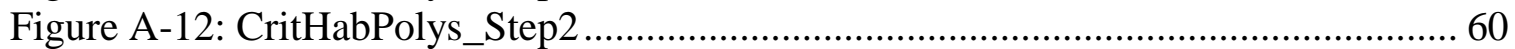

Figure A-13: CritHab_Step2_background_ES (background process).............................. 61

Figure A-14: CritHab_Step2_background_TS (background process)............................... 62

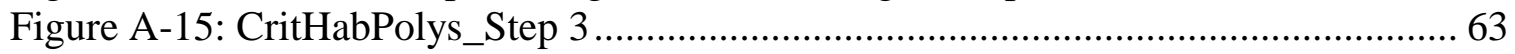

Figure A-16: CalcSpList Script Tool (Simplifying_Sp_List_Final.py) used in Step_3 ... 64

Figure A-17: Calculate Endangered Species Count Script Tool used in Step_3

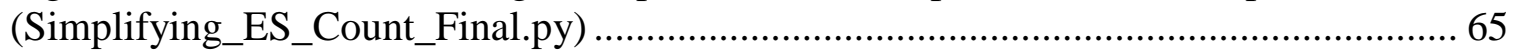

Figure A-18: Calculate Threatened Species Count Script Tool Used in Step_3

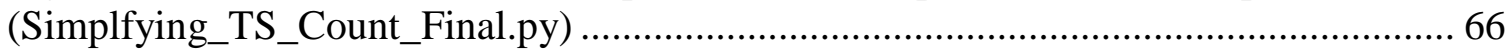

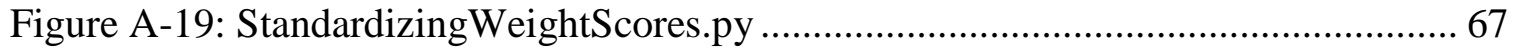

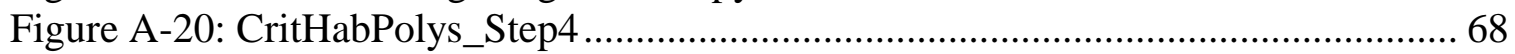

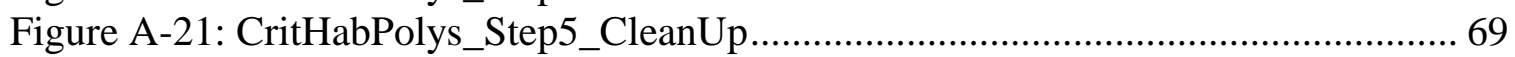

Figure A-22: User Interface for CritHabPolys_Step1 and CritHabPolys_Step2 .............. 69

Figure A-23: User Interface for CritHabPolys_Step3 and CritHabPolys_Step4 ............... 70

Figure A-24: User Interface for CritHabPolys_Step5_CleanUp ....................................... 71

Figure A-25: Important Bird Areas Data Prep Toolset.................................................. 71

Figure A-26: Important Bird Areas Data Prep Step 1 ……........................................... 72

Figure A-27: Important Bird Areas Data Prep Step 2................................................ 72

Figure A-28: User Interface for ImportantBirdAreas Step1 and ImportantBirdAreas Step

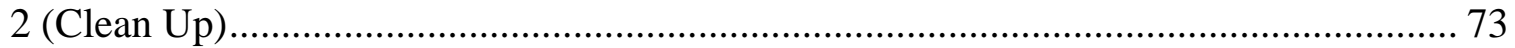

Figure A-29: Wetland Prioritization Analysis .............................................................. 73

Figure A-30: Prioritization Analysis Step 1................................................................. 74

Figure A-31: Prioritization Analysis Step 2 (Optional but Recommended)..................... 74

Figure A-32: Prioritization Analysis Step 3 ............................................................... 74

Figure A-33: Prioritization Analysis Step 4............................................................... 75

Figure A-34: BackgroundProcess_Prioritization Script Tool

(CalculateFinalScore_Pro_Version2.py) rows 1-34 …….............................................. 75

Figure A-35: BackgroundProcess_Prioritization Script Tool

(CalculateFinalScore_Pro_Version2.py) rows 34-70 ………........................................ 76

Figure A-39: Parameters used to Integrate CalculateFinalScore_Pro_Version2.py into a

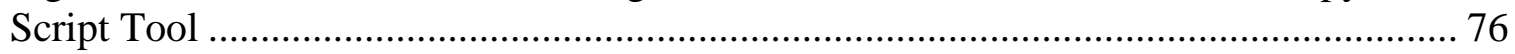

Figure A-40: Wetland Prioritization Toolbox for ArcMap 10.5 ……………………...... 77

Figure A-41: PrioritzeWetlands_Web_Version2d......................................................... 77

Figure A-42: CalculateFinalScore_Web_Version2.py Rows 1-35 ................................... 78

Figure A-43: CalculateFinalScore_Web_Version2.py Rows 35-67.............................. 79

Figure A-44: CalculateFinalScore_Web_Version2.py Rows 67-100............................... 80

Figure A-46: When publishing the service, input should be "Constant value"................ 81

Figure A-47: Output_Layer_Name parameter should not be visible, when configuring the geoprocessing tool 


\section{List of Tables}

Table 3-1. Summary of Project Requirements..................................................... 9

Table 4-1. Data Sources for the Project ................................................................... 15

Table 5-1. Data Preparation Steps for Important Bird Areas Dataset.......................... 18

Table 5-2. Data Preparation Steps for Critical Habitat Polygons Dataset ..................... 19

Table 5-3. Example Attribute Table After Union for Two Unique Species Ranges ..... 22

Table 5-4. Data Preparation Steps for Critical Habitat Lines (Rivers) ......................... 23

Table 5-5. Wetland Data Preparation (Prioritization Analysis Step 2) ........................ 25

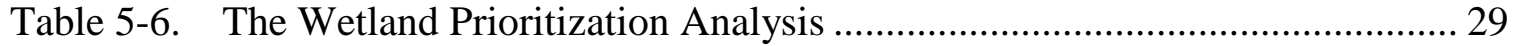

Table 5-6. Fields in Attribute Table of Union-ed Feature Class from Step 1............... 30

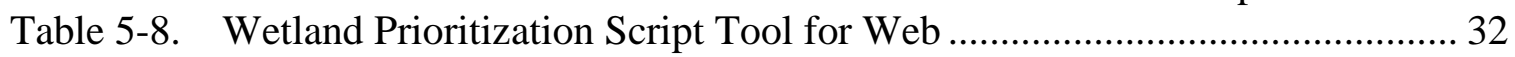

Table 6-1. Distance Areas and Thresholds in the Analysis ...................................... 43 



\section{List of Acronyms and Definitions}

$\begin{array}{ll}\text { FR } & \text { Functional requirement } \\ \text { GIS } & \text { Geographic Information Science } \\ \text { IBA } & \text { Important Bird Area } \\ \text { NFR } & \text { Non-functional requirement } \\ \text { NWI } & \text { National Wetlands Inventory (part of the U.S. Fish and Wildlife Service) } \\ \text { OID } & \text { Object ID } \\ \text { USFWS } & \text { U.S. Fish and Wildlife Service }\end{array}$





\section{Chapter 1 - Introduction}

In environmental and conservation fields, managers and other decision makers need to prioritize their efforts to specific areas using multiple-criteria decision analysis, to maximize environmental protection given limitations of budget and time. However, creating these prioritization models requires a combination of both scientific and technical skills, and many of those with the expertise to create scientifically sound prioritization models have limited time to devote to the technical aspects of the analysis. There was a need to automate this analysis process to enable scientists and other decision makers to quickly repeat analyses with different criteria and compare the results.

This chapter will give an overview of the client of this project and problem this project addressed. The chapter will then briefly discuss the solution that was proposed for this project and the audience this project was geared towards.

\subsection{Client}

The client of this project was Cade London, Policy Advisor for International Affairs for the U.S. Fish and Wildlife Service (USFWS), and part of his job responsibilities is to determine wetlands that should be considered as Ramsar wetlands of "international importance" (C. London, personal communication, 15 Oct 2017). The client's main responsibilities for this project included providing domain knowledge, such as what important prioritization criteria should be considered, and providing the relevant datasets.

\subsection{Problem Statement}

In 1975, the United States signed an international agreement on the protection of wetlands - "The Ramsar Convention on Wetlands of International Importance Especially as Waterfowl Habitat" - and this project's client holds responsibility for designating Ramsar wetlands in the United States. Historically, the process for designating a wetland as a Ramsar wetland of international importance has involved a lot of paperwork, and there has been no clear way for the client to decide which wetlands are more important than others. Because of this, the client wanted to use geographic information science (GIS) to prioritize wetlands in the United States in such a way that he could compare the wetland priorities of different groups and individuals, in order to make more informed decisions when designating Ramsar wetlands (C. London, personal communication, 2017). However, creating a wetland prioritization analysis for even one person's priorities required a unique combination of both scientific and technical skills, and the client and his associates had little time to devote to this effort.

\subsection{Proposed Solution}

There was a need to automate the prioritization of wetlands with GIS such that scientists and decision-makers could quickly complete the wetland prioritization analysis and compare wetland priorities without requiring an extensive knowledge of GIS. This project fulfilled this need by automating the analysis process and creating a web application so that users could prioritize wetlands according to their own priorities. With 
the analysis process simplified, decision-makers can easily prioritize wetlands according to their views and then compare those priorities with those of other stakeholders.

\subsubsection{Goals and Objectives}

The goal of this project was to automate a pilot wetland prioritization analysis in California so that the client and his associates could re-do wetland prioritization analyses in a multiple-criteria decision analysis framework, where the opinions of different stakeholders are taken into account. The project consisted of two main objectives that worked to meet this goal. The first was to automate the prioritization analysis in such a way that the GIS analysis could be quickly repeated with different weights for the various factors in the analysis. The second objective of this project was to develop a web application where users could prioritize wetlands and compare the results without needing a technical background in GIS.

\subsubsection{Scope}

The scope of the implementation included two deliverables. The first deliverable was an ArcGIS ModelBuilder toolbox that enabled the client (or his associates) to re-do the wetland prioritization analysis in ArcGIS Pro, as well as to update the analysis with newer versions of the input data. This toolbox consisted of model and script tools organized into toolsets within the toolbox, and contained both toolsets used to prepare the input data for analysis, as well as a toolset used to perform the prioritization analysis.

The second deliverable was a custom web application where people could specify different weights of importance for the various factors that were used in the analysis, and generate a map showing wetland priorities according to their personal weighting scheme. The map layer generated by the web application could then be saved and compared with other peoples' maps of wetland priorities, so that the client could gain an understanding of how priorities compare between several decision makers.

\subsubsection{Methods}

The custom toolbox created as part of this project was created using Model Builder in ArcGIS Pro to create custom models and script tools organized into toolsets within the toolbox. Wing IDE 1016.0 was also used to write the Python scripts that were used in the script tools. ArcMap was used to create the model that was used for the geoprocessing service, as well as to publish the geoprocessing service. The web application was created on the University of Redlands server using Esri's Web AppBuilder online.

\subsection{Audience}

The audience being addressed by this project are primarily scientists and decision-makers in the environmental field who will have a range of experience with GIS (from little or limited knowledge of GIS to extensive knowledge). 


\subsection{Overview of the Rest of this Report}

The format of the rest of this report goes as follows. Chapter 2 details the background of this project and a literature review that was conducted. Chapter 3 outlines the systems analysis and design. Chapter 4 outlines the database design of the project (both conceptual and logical data model) as well as the data sources for the project. Chapter 5 describes the implementation of this project and Chapter 6 describes the results and an analysis of those results. Chapter 7 contains conclusions from this project and suggestions on future work. At the end of this report are the Works Cited section, and an Appendix providing diagrams of the models, scripts, and script tools created as part of this project. 



\section{Chapter 2 - Background and Literature Review}

The chapter provides brief literature reviews on a few topics that were pertinent background information for this project. The chapter begins with a literature review that was conducted on previous efforts in wetland prioritization, and the criteria used by scientists to prioritize wetlands. A short literature review is then given on multiple criteria decision analysis, as that was a key concept for how the client of this project envisioned using the final products from this project. A brief discussion on buffer distances delineating the extent of movement of wetland-dependent animals is then presented, to inform decisions made in the analysis process on buffer distances. This chapter ends with a miscellaneous note on the decision to perform this project as a vector (instead of raster) overlay.

\subsection{Wetland Prioritization - Previous Efforts and Criterion for Prioritization}

Wetlands are important habitat for conservation efforts. Not only do they play an important role in providing habitat to many species (Copeland et al., 2010), but they also provide a plethora of services to humans such as controlling erosion (Ming, Xian-guo, Lin-shu, Li-juan, \& Shouzhenga, 2007) and providing important flood protection along rivers (McInnes, 2016; Ming et al., 2007). There is a great importance to being able to prioritize conservation areas scientifically, instead of going about it in random or ad-hoc ways (C. London, personal communication, 16 Oct 2017; Prendergast, Quinn, \& Lawton, 1999). But, prioritizing these areas is not a simple and straightforward task, for there are many different factors that contribute to the relative importance of any one area over another.

Using GIS to automate a wetland prioritization analysis such that users are given a list of potential criterion to use in the analysis and are allowed to choose their own weights of importance for the various factors is an effective way to begin to address these problems, by simplifying the prioritization analysis into an interface that both scientists and land managers can use to integrate their specialized knowledge into an analysis, and compare the results with one another.

This section will discuss various criterion that could be considered for wetland prioritization and will highlight previous work that has been done to use GIS to help prioritize wetlands and conservation areas.

\subsubsection{Criterion for Wetland Prioritization}

One criterion that is often used to prioritize areas for conservation is "biodiversity," or, the diversity of species in an area (Prendergast et al., 1999). This is often hard to measure though. Another criterion scientists sometimes use is "indicator species," which are species that give an indication of the overall health of an ecosystem (Andersen, Hoffmann, Muller, \& Griffiths, 2002; Prendergast et al., 1999). A third criterion that can be used in the United States, is the presence or absence of endangered species, assuming the spatial ranges of those species are known (C. London, personal communication, 16 Oct 2017). 
Because taking into account the ranges of many different species can be difficult and time consuming, the concept of umbrella species is an attractive alternative (Roberge \& Angelstam, 2004). According to Roberge \& Angelstam (2004), "An umbrella species is defined as a species whose conservation is expected to confer protection to a large number of naturally co-occurring species" (p. 76). Oftentimes one "umbrella species" is not sufficient to protect all the diversity in an area, giving support to the "multi-species" approach where the ranges of multiple umbrella species are considered when prioritizing conservation area (Roberge \& Angelstam, 2004). In the case of wetlands, various plants and dragonflies have been suggested as potential umbrella species (Bried, Herman, \& Ervin, 2007). There are many other criteria that can be considered when prioritizing wetlands to conserve, such as considering areas that are important for migratory birds (Copeland et al., 2010; United Nations Educational Scientific and Cultural Organization, 1994).

As a final note, it should also be noted that there is sometimes a disconnect between land managers and scientists (Arlettaz et al., 2010; Kiester et al., 1996; Prendergast et al., 1999; Thorhill, 2014), so priorities of scientists in the academic field may not be the same as priorities of land managers and other decision makers in the field.

\subsubsection{Previous Work on Prioritizing Wetlands with GIS}

This is not the first time that someone has attempted to construct a geospatial model to prioritize wetlands. For example, Copeland et al. (2010) discussed a geospatial analysis they had conducted in Wyoming. In their study, they mapped areas with high wetland density and then assessed which wetlands had the highest priority by overlaying that data with spatial data that showed the biodiversity, condition, and vulnerability of those wetlands, as well as the use of those areas for agricultural and recreational use. In addition, one of the things the Copeland article mentioned was what definition they used for wetlands. This is an important thing to note, because according to this project's client, wetlands are often defined very broadly and can consist of several different ecoregions.

\subsection{Multiple Criteria Decision Analysis and GIS}

As multiple criteria decision analysis was an important function that this client felt this project outcome could assist in, a brief literary review on multiple criteria decision analysis and its integration with GIS was conducted.

An article from 2001 states that multiple criteria analysis methods "effectively decompose choice decisions into evaluations of sets of criteria that allow the performance of alternatives to be judged. Through this process of decomposition, elements of agreement and conflict between different individuals and groups can be exposed and debated" (Feick \& Hall, 2001, p. 14). An article published in 1995 also specified that multiple criteria decision analysis should consist of choosing from a small to moderate set of choices (Jankowski, 1995), rather than an unlimited set of choices.

To see if this project fulfilled the need posed by a multiple criteria decision analysis framework, the project was compared against a definition of that framework. According to Jankowski (1995), structured decision situations can be split into four steps: 1) problem definition, 2) search for alternatives and selection criteria, 3) evaluation of alternatives, and 4) selection of alternatives. To add on the qualifications of multiple 
criteria decision analysis as specified by Jankowski, the list of alternatives created in step 3 should also be small to moderate.

Applying the steps listed above to this project, Step 1 was completed when the client defined the problem. Searching for alternatives and selection criteria (Step 2) was the process of working with the client to define the different factors to use in the analysis. The tools and web application created by this project enable the evaluation of alternatives (Step 3). Narrowing down the alternatives and selecting the alternatives (Step 4), is not completed by this project, nor should it be, for the client is the one with the information and domain knowledge needed to refine the options presented. This project only aims to help create alternative solutions. Thus, while this project is not relevant to perhaps all aspects of multiple criteria decision analysis, it can be concluded that this project is valuable for particular steps in the multiple criteria decision analysis framework.

Multiple criteria decision analysis has been integrated with GIS before. One example of this was published in 2000. That example dealt with land use and allowed users to explore different opinions on how to allocate land use in a tourist area. According to the article, the goal of that project was to integrate GIS with multiple criteria analysis, to "assist individuals and groups to explore alternative development strategies" (Feick \& Hall, 2000). This is another example of GIS being used to help individuals explore options and differing opinions in a spatial context. That project however, dealt with real estate and land use designations, while this project dealt with determining wetland priority areas.

\subsection{Appropriate Buffer Distance for Wetland Prioritization}

A brief literature review was also conducted to determine average distance thresholds of wetland-dependent species movement, in order to decide on an appropriate buffer distance to use for threshold distance buffers in the analysis.

In a paper about wetland-dependent reptiles, a review of the literature showed that 499-1518 meters was a mean minimum and maximum distance range for several reptile species from different taxonomic groups when moving between wetlands, and that a 375 m "terrestrial core zone" would "encompass [...] 95\% of terrestrial habitats used by $[\ldots$ Chelodina] longicollis," the turtle species of focus in the study (Roe \& Georges, 2006).

Another literature review of wetland-dependent reptiles and amphibians, concluded that the size of terrestrial habitat that surrounded wetlands where these species were found ranged from 127 to 290 meters (Semlitsch \& Bodie, 2003). This paper suggested that an aquatic buffer and core habitat designation, as well as a terrestrial buffer distance be applied to wetlands in order to better protect the core habitats of these species and minimize edge effects (Semlitsch \& Bodie, 2003). Adding the recommended aquatic buffer and core habitat ranges with the recommended terrestrial buffer range, the total recommended buffer size from the water's edge was a distance that ranged between 192 to 339 meters (Semlitsch \& Bodie, 2003).

The third paper reviewed here focused on buffer zones necessary to maintain distance between waterfowl and motorboats, in order to avoid scaring the birds and disrupting their life cycles (Rodgers \& Schwikert, 2003). According to this paper, flush distances, i.e., the distance from a motor boat at which the bird flew away, for 13 species of water birds ranged from 49 to 172 meters, and the recommended buffer zones from 
this study to minimize disturbance to birds ranged from 130 meters to 365 meters, depending on the species (Rodgers \& Schwikert, 2003).

This literature review on buffer distances was in no ways complete or sufficient for a strong scientifically valid analysis. However, it was sufficient to gain an understanding of the general scale that a reasonable buffer distance would have (tens, hundreds, or thousands of meters) and to come up with a serviceable example distance to use in the analysis - 500 meters - since no other distances were provided by the client or his associates.

\subsection{Miscellaneous Note on Vector vs. Raster Overlay Research}

For this project, it was ultimately decided to perform the analysis as a vector overlay. When deciding this, two main factors were considered. First, the wetland data layer provided by the National Wetlands Inventory, which was the basis for this analysis, was already in vector format and was at a particularly detailed scale. In addition, other datasets, such as threatened and endangered species, rivers, and important bird areas, were also provided in the vector format. Converting the wetland data to raster format would have compromised the accuracy of the wetland shapes-particularly small shapes, as there were many extremely small wetland features in the dataset. Second, the input data was maintained in vector format in the interest of getting the highest cartographic output, with natural looking shapes. In the end, the wetland shapes were compromised to a small degree when using the "Aggregate Polygon" tool to simplify the data layer, but the results were acceptable as the error introduced can be controlled. As such, the approach of vector analysis was adopted in this project.

\subsection{Summary}

In conclusion, the literature reviews completed above provided important background information for this project. The wetland prioritization background literature review gave important context for discussing priorities with the client. The review on multiple criteria decision analysis and its relation to GIS analyses provided important support that the project would, in fact, be useful for multiple criteria decision analysis as was supposed by the client. Finally, the literature review on buffer distances directly led to the decision to

use 500 meters as the threshold buffer distance in this analysis. Chapter 3 will discuss the systems analysis and design of this project. 


\section{Chapter 3 - Systems Analysis and Design}

This chapter will discuss the systems analysis and design for this project. It will first discuss the client's problem that was addressed, the requirements analysis that was completed, and the system design of the project. This chapter will then briefly discuss the project plan and what changes were made to it.

\subsection{Problem Statement}

In environmental and conservation fields, managers and other decision makers need to prioritize their efforts to specific areas using multiple-criteria spatial analysis, to maximize environmental protection given limitations of budget and time. However, creating these prioritization models requires a combination of both scientific and technical skills, and many of those with the expertise to create scientifically sound prioritization models have limited time to devote to the technical aspects of the analysis. This is particularly true when the decision makers must iterate through many alternative models. As such, the problem that this project addressed was to develop an automated workflow that enables scientists and other decision makers (who may have limited GIS experience), to quickly repeat analyses with different criteria and compare the results.

\subsection{Requirements Analysis}

The requirements of this project are best described in relation to the main deliverables of the project. The first deliverable was a ModelBuilder Toolbox with models and script tools (organized in toolsets), that condensed the prioritization analysis into an easily repeated form and allowed the input of new data layers and weights into the analysis. The second deliverable was a custom geoprocessing service that allowed the ranking of wetlands according to users-defined weights for the various factors in the wetland prioritization analysis. The third deliverable was a custom web application to host the geoprocessing service. Table 3-1 summaries the functional and non-functional requirements of each deliverable.

Table 3-1. Summary of Project Requirements

\begin{tabular}{|l|l|}
\hline Deliverable & Requirement \\
\hline \multirow{4}{*}{$\begin{array}{c}\text { Model Builder } \\
\text { Toolbox }\end{array}$} & $\begin{array}{l}\text { The tools all work properly (as expected and as } \\
\text { desired) [Functional requirement] }\end{array}$ \\
\cline { 2 - 3 } & $\begin{array}{l}\text { The tools all produce consistent results } \\
\text { [Functional requirement] }\end{array}$ \\
\cline { 2 - 3 } & $\begin{array}{l}\text { The tools are organized in a self-explanatory } \\
\text { manner [Non-Functional requirement] }\end{array}$ \\
\hline
\end{tabular}




\begin{tabular}{|c|l|}
\hline Deliverable & Requirement \\
\hline \multirow{5}{*}{$\begin{array}{c}\text { Geoprocessing } \\
\text { Service }\end{array}$} & $\begin{array}{l}\text { The tools included sufficient documentation } \\
\text { and metadata (including explanatory } \\
\text { descriptions for each parameter in all the tools) } \\
\text { such that the tools could be used by GIS } \\
\text { analysts without outside assistance [Non- } \\
\text { Functional Requirement] }\end{array}$ \\
\hline \multirow{5}{*}{$\begin{array}{l}\text { The geoprocessing service worked consistently } \\
\text { Aptom Web }\end{array}$} & $\begin{array}{l}\text { Users can specify weights of importance for the } \\
\text { different input factors (i.e. Endangered Species, } \\
\text { Important Bird Areas etc.) [Functional } \\
\text { Requirement] }\end{array}$ \\
\cline { 2 - 3 } & $\begin{array}{l}\text { 2) Results from user-ran analyses can be saved } \\
\text { externally [Functional Requirement] }\end{array}$ \\
\cline { 2 - 3 } & $\begin{array}{l}\text { Results are automatically symbolized with } \\
\text { effective pre-defined symbology [Functional } \\
\text { Requirement }\end{array}$ \\
\hline
\end{tabular}

The major functional requirement of the ModelBuilder Toolbox was that the tools all worked properly and produced consistent results. Non-functional requirements were that the tools were organized in a self-explanatory manner, and that they included sufficient documentation and metadata such that the tools could be used by GIS analysts without outside assistance. The geoprocessing service had the functional requirement that it worked consistently. It also had the non-functional requirement that it would complete the analysis in a minute or faster. The web application had four requirements, three of them being functional requirements and one non-functional. The first two functional requirements were that users could specify weights of importance for different layers and that the results can be saved externally. The next requirement was that users are asked to specify an area of extent. This is a necessary functional requirement, in order to ensure that data processing does not take too long when users use the web tool. Effective, predefined symbology of output results was another functional requirement for the web tool to ensure that results were pre-generated in easy to understand symbology that was consistent across different users of the web application and that clearly communicated the results of the analysis.

\subsection{System Design}

The system design can be described as follows and as depicted in Figure 3-1. 


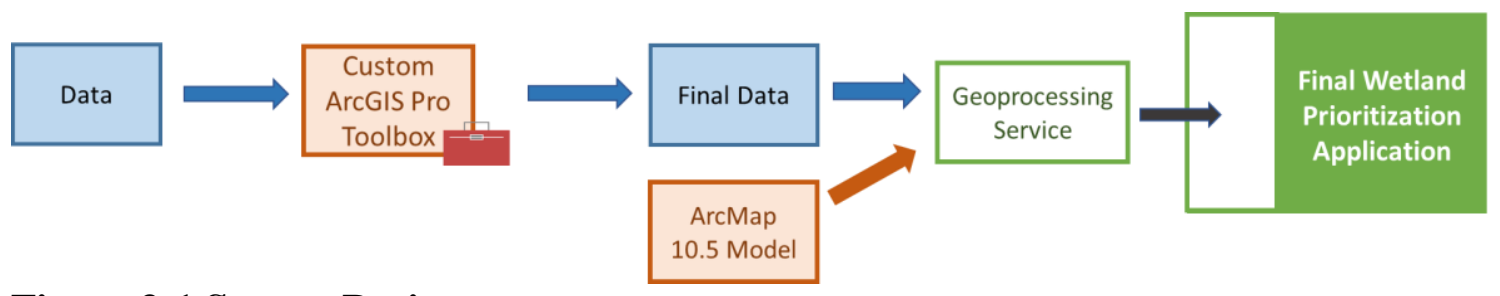

\section{Figure 3-1 System Design}

There are 6 major components that were included in the system design. The "Data" component served as the basis for the project and was used in the creation of all other components of the project. The second component, "Custom ArcGIS Pro Toolbox," was modeled around the input "Data" and consisted of models and script tools. This custom toolbox then processed the "Data" to create the "Final Data." An "ArcMap 10.5 Model" then processed the "Final Data" again to create a "Geoprocessing Service," that was imbedded into the "Final Wetland Prioritization Application," which was a custom web application created in Web AppBuilder.

\subsection{Project Plan}

The original project plan is described in Figure 3-2. The plan was divided into four phases, including Data Gathering, Analysis, Geoprocessing Tool Development, and Web Interface Development.

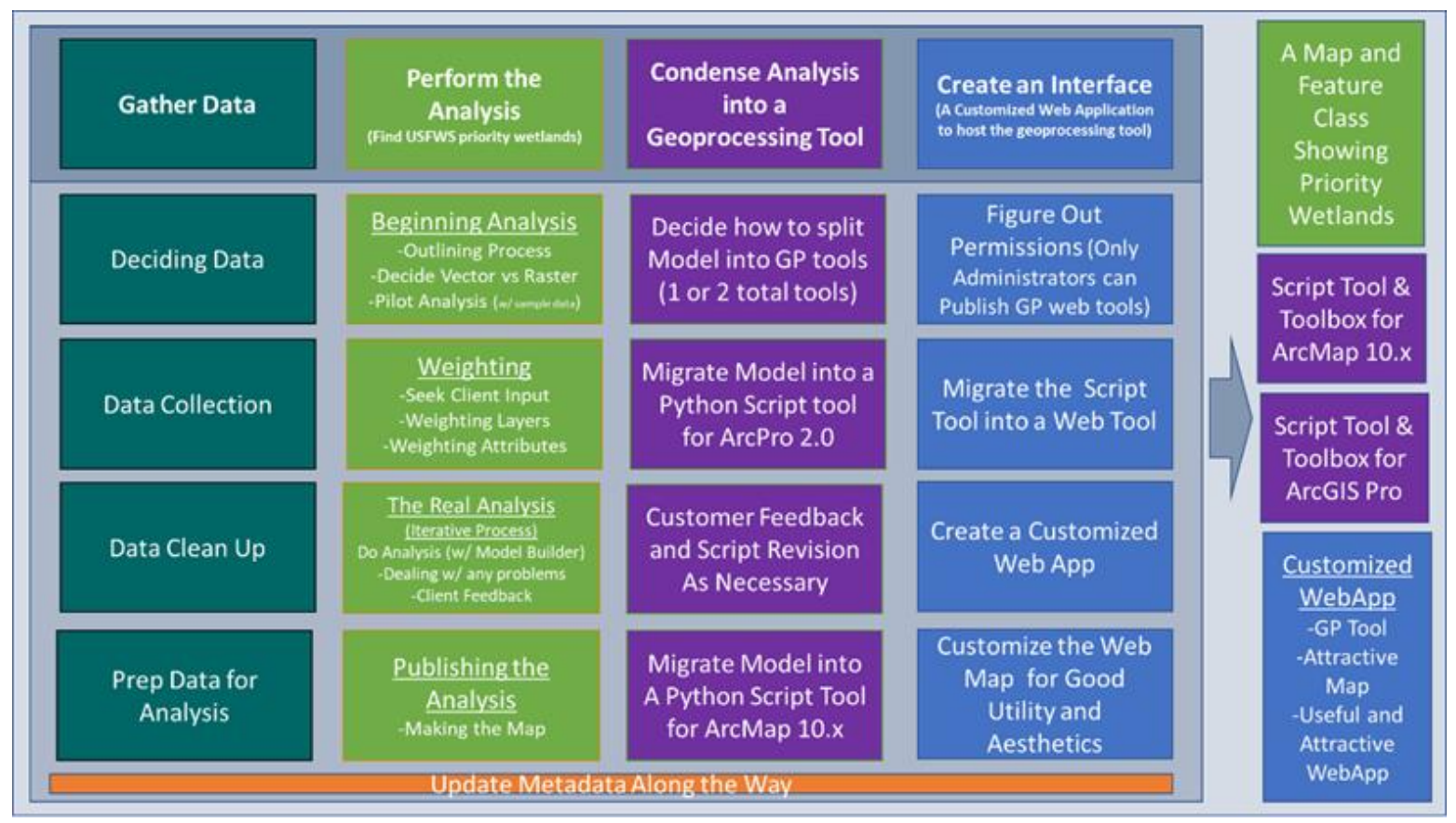

Figure 3-2 Project Plan

Four deliverables were expected from this project plan: 
1) A collection of hardcopy maps and one web map depicting priority wetlands in California according my client's interpretation of USFWS priorities;

2) A ModelBuilder toolbox for ArcGIS Pro containing the model and script tools used in the analysis;

3) A ModelBuilder toolbox for ArcMap 10.x containing the model and script tools used in the analysis;

4) A ModelBuilder toolbox for ArcMap 10.x containing the model and script tools used in the analysis, and a custom web application with a geoprocessing service embedded in it.

During the project progression, the project plan was reviewed and modified. One important alteration to the plan was the final priority maps were not produced from the analysis due to the lack of domain knowledge. Instead, the analysis was implemented as a set of tools that allows the client to create his own maps. The second change was that the toolbox was expanded to include the data preparation steps as well as the final overlay analysis steps for the prioritization analysis. This will benefit the client in that data scrubbing was a tedious process and automating the data preparation process will reduce the errors.

\subsection{Summary}

In conclusion, to help the USFWS compare wetland priorities in California, a set of geoprocessing tools was created which automated the wetland prioritization analysis in ArcGIS Pro, a geoprocessing service was then created and embedded in a web application, allowing users to prioritize wetlands according to their own priorities. A help document was also created for the USFWS, accompanying these deliverables. The project plan did change as the MIP progressed, but all the necessary requirements (listed in the Requirements Analysis section) were completed. The next chapter will discuss more about the data that was collected and the final database design for the project. The chapters after that will then discuss in greater detail the 'GIS details' of this project. 


\section{Chapter 4 - Database Design}

This chapter will review the conceptual and logical data models for this project. It will then discuss the data sources and data collection methods of the project. Traditionally, data scrubbing and loading would also be included in this chapter, but since the data preparation - and automating it — was such a large and important part of this project, the data preparation will be more fully discussed in chapter 5, and only briefly touched upon in this chapter (in the logical data model section).

\subsection{Conceptual Data Model}

The conceptual model of a client's problem domain summarizes the entities of interest and their relationships (Douglas Flewelling, personal communication). Figure 4-1 displays the conceptual model for this project, with its associated entities of interest and their relationships.

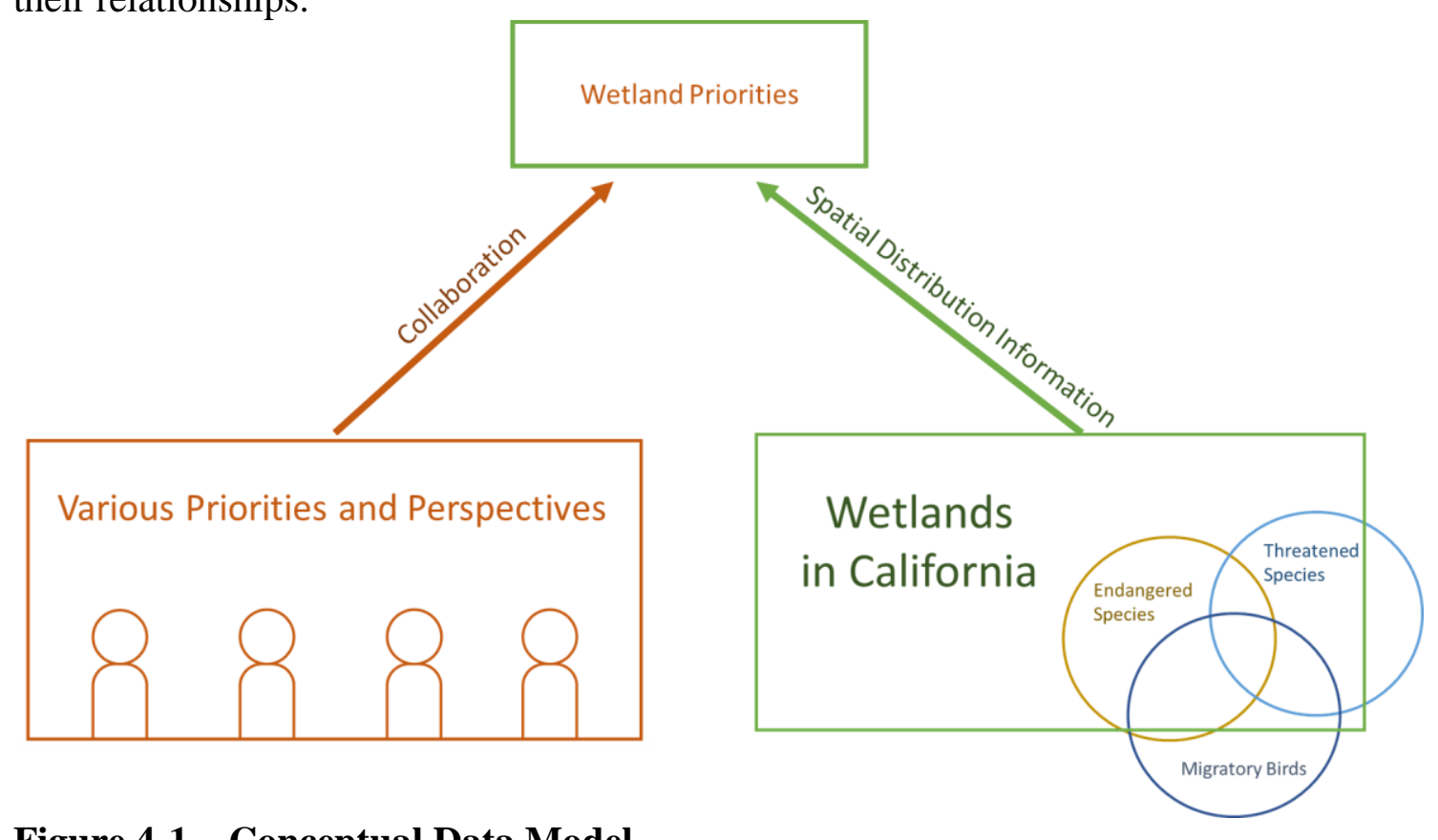

Figure 4-1 - Conceptual Data Model

The main entities of interest for my client were wetlands, as well as any additional any entities whose spatial distribution and attributes could be used to help prioritize wetlands from the perspective of the USFWS. The final entities incorporated into the analysis were those which passed the following four checkpoints:

1) were identified by the client,

2) related to published priorities of the USFWS (US Fish and Wildlife Service, 2013)

3) were easily represented by available data and

4) were able to be integrated into the analysis before the passing of the data collection deadline. 
Entities that were ultimately integrated into the analysis (in addition to wetlands), were endangered species, threatened species, and migratory birds.

With the creation of the web application described in this project, the spatial distribution of habitat for those entities of interest can now be integrated with the different opinions of various stakeholders (acquired through collaboration). Altogether, the integration of all this information allows one to determine wetland priorities in California.

\subsection{Logical Data Model}

The logical model for this project's database can be summarized in terms of input "wetland prioritization factors" and output data. All the output data was stored in a single geodatabase, except for the raster dataset which was simply stored as a raster file. The diagram below summarizes the logical data model of the project (Figure 4-2).

Input Data Output Data

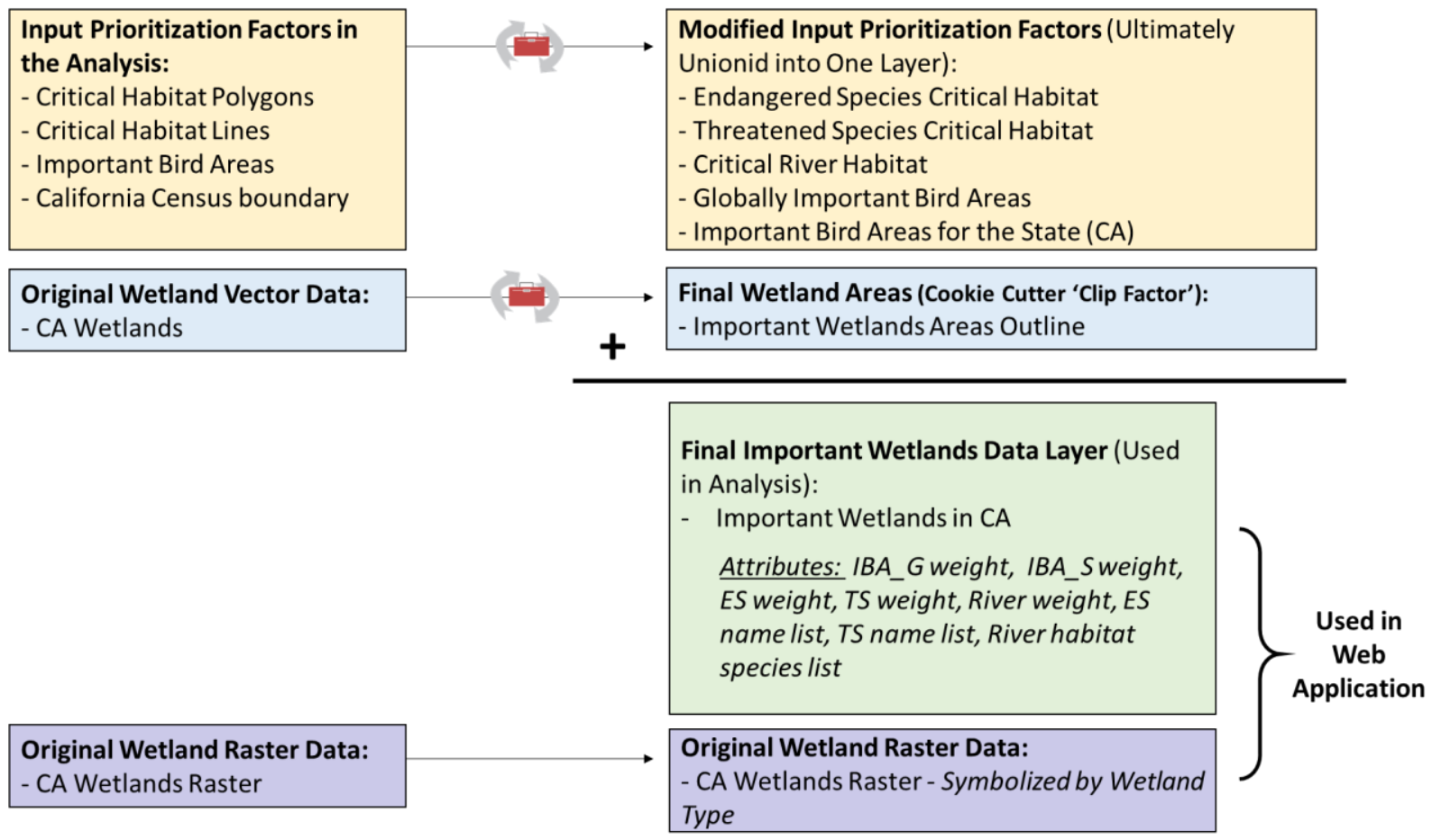

Figure 4-2: Logical Data Model

Input data layers used in this project consisted of wetland data for California (in both vector and raster form) and wetland "prioritization factors," including critical habitat polygons and lines for wetland-dependent endangered and threatened species, and important bird areas. The California boundary from the U.S. Census Bureau was another input dataset, used to clip wetland prioritization layers to California.

Since the final analysis would consist of giving weights to the different prioritization factors, each prioritization factor layer was made into a separate, distinct layer. Thus, the critical habitat polygons were divided into two layers: one layer for endangered species habitat and the other for threatened species habitat. Similarly, important bird areas were 
split into two layers depending on their status as "Global" or "State" Important Bird Areas. The critical habitat lines displayed important river systems for two species of fish, Chinook Salmon and Steelhead Trout, and were kept as one layer. All these prioritization factor layers were then put into one combined prioritization layer.

The wetlands data was also modified to allow a fast upload to the web application. The detailed process will be described in Chapter 5. That simplified wetlands layer was then used to clip the combined prioritization layer. The clipped output of this step was the final feature layer which was used in the wetland prioritization analysis.

That final analysis data layer, as well as the raster version of the original wetland data, was then integrated into the web application where users would be able to choose their own weights of importance for the various factors in the analysis and produce their own copy of the final analysis layer, symbolized by their custom priorities.

\subsection{Data Sources}

The data sources of various data layers are described in Table 4-1. Wetland data were provided by the National Wetlands Inventory (NWI), which is part of the U.S. Fish and Wildlife Service (USFWS). Critical Habitat lines and polygons for wetland-dependent species were also provided by the NWI. This data originally came from ecos.fws.gov, but the NWI went through the data and selected out only the critical habitat datasets for species that depended on wetland or deep-water habitat for at least one part of their life cycle.

\section{Table 4-1. Data Sources for the Project}

\begin{tabular}{|l|l|l|l|}
\hline $\begin{array}{l}\text { Data Type } \\
\text { (Name) }\end{array}$ & $\begin{array}{l}\text { Attributes Included in } \\
\text { Final Analysis }\end{array}$ & Format & Data Source \\
\hline Wetlands & $\begin{array}{l}\text { - Only location data saved } \\
\text { for the analysis } \\
- \text { Wetland type displayed on } \\
\text { the web application }\end{array}$ & $\begin{array}{l}\text { Vector } \\
\text { (Polygon) and } \\
\text { Raster }\end{array}$ & $\begin{array}{l}\text { National } \\
\text { Wetlands } \\
\text { Inventory (NWI) }\end{array}$ \\
\hline $\begin{array}{l}\text { Critical Habitat } \\
\text { (Lines and } \\
\text { Polygons) }\end{array}$ & $\begin{array}{l}\text { - Endangered or Threatened } \\
\text { Status } \\
- \text { Species names } \\
\text { (species count calculated) }\end{array}$ & $\begin{array}{l}\text { Vector (Polygon } \\
\text { and Line) }\end{array}$ & NWI \\
\hline $\begin{array}{l}\text { Important Bird } \\
\text { Areas }\end{array}$ & $\begin{array}{l}\text { - Type (Global or State) } \\
\text { CA State }\end{array}$ & $\begin{array}{l}\text { Vector } \\
\text { (Polygon) }\end{array}$ & $\begin{array}{l}\text { Autional } \\
\text { Socioty }\end{array}$ \\
\hline Boundary & Sonalysis extent) & $\begin{array}{l}\text { United States } \\
\text { Census Bureau }\end{array}$ \\
\hline
\end{tabular}

Important Bird Area data was difficult to find. Originally, migratory bird flyways and stopover locations were searched for, but the search for this data resulted in no good datasets. Finally, data on important bird areas in California were obtained from the National Audubon Society after a data agreement form was signed. 
The last data layer used in the analysis was the CA State Boundary for 2010 from the United States Census Bureau. This data layer was used to clip extraneous features from the critical habitat data.

\subsection{Summary}

In conclusion, this project brought together data on the distribution of wetlands, endangered species habitat, threatened species habitat, and important bird habitat in such a way that it could then be integrated with the different opinions of various stakeholders. The input data was manipulated such that each factor of importance was represented in a separate layer, and those layers were then all combined with the "Union" tool in ArcGIS Pro such that an output data layer was created where all unique areas of overlap were identified, so that a custom priority index score could then be calculated based on userdefined weights. The output data layer was also clipped to a simplified version of the original polygon wetland data. That final output layer and a raster layer showing the original wetland data (symbolized by wetland type), were the outputs used in the final web application. Data to be integrated into the analysis were decided upon through collaboration between myself and Cade London from the USFWS, and through referencing the published priorities of the USFWS (US Fish and Wildlife Service, 2013). Data sources include the USFWS and the National Audubon Society. 


\section{Chapter 5 - Implementation}

This project created a ModelBuilder Toolbox in ArcGIS Pro with models and scripts automating both the final wetland prioritization analysis as well as the data preparation necessary for the analysis, so that the U.S. Fish and Wildlife Service (USFWS) could repeat the analysis for different areas and with updated data. Automating the process also reduced errors that could happen during the GIS analysis process, such as performing the analysis in an unsuitable projection.

This chapter starts by giving some brief explanation about the organization and use of the Wetland Prioritization toolbox. It then gives an overview of the data preparation necessary for each factor in the analysis as well as an overview of the subsequent models that were created to automate that data preparation. It then discusses the toolset created for the wetland prioritization analysis, the transferring of the last step of that toolbox into a geoprocessing service, and the final web application created to host the geoprocessing service.

\subsection{Introduction to Wetland Prioritization Toolbox}

All the models for the analysis were saved in one ArcGIS Pro Toolbox called "Wetland_Prioritization Toolbox". The Wetland Prioritization toolbox consists of four toolsets (Figure 5-1).

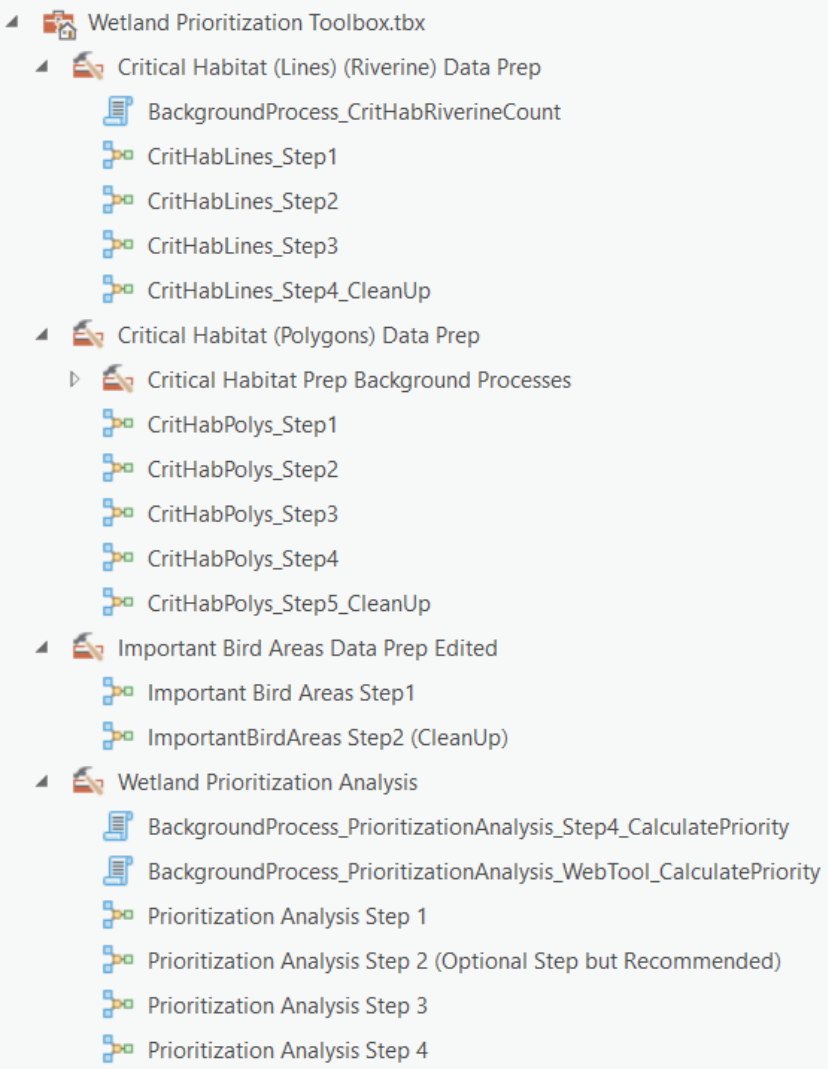

Figure 5-1: Toolsets, Models, and Script Tools within Wetland Prioritization Toolbox 
The first three toolsets, Critical Habitat (Lines) (Riverine) Data Prep, Critical Habitat (Polygons) Data Prep, and Important Bird Areas Data Prep, prepare the datasets from the USFWS and the National Audubon Society for use in the analysis. The fourth toolset, Wetland Prioritization Analysis, completes the wetland prioritization analysis. The following sections will explain each model and script included in these four toolsets.

\subsection{Important Bird Areas Data Preparation and Automation}

The Important Bird Area data was provided by the National Audubon Society. The original attribute table for the data included a column named "PRIORITY" where features were labeled for what type of priority they were. In California (at the time of data download), there were only two priority levels present, Global and State. All other attributes were removed for this pilot analysis.

Table 5-1 shows the complete workflow of the "Important Bird Areas Data Prep" toolset, which includes two important steps, each implemented by an independent model.

Table 5-1. Data Preparation Steps for Important Bird Areas Dataset

\begin{tabular}{|c|c|c|}
\hline \multirow{7}{*}{ 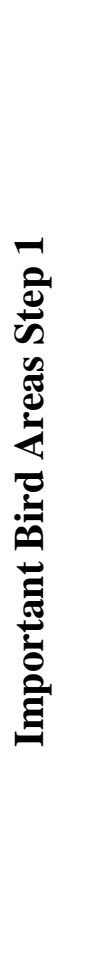 } & Operation Performed & Reasoning / Explanation \\
\hline & Project & $\begin{array}{l}\text { Projected the data into the same } \\
\text { projection used by all the other } \\
\text { data in the analysis, NAD } 1983 \\
\text { Albers Equal Area }\end{array}$ \\
\hline & Make Feature Layer & $\begin{array}{l}\text { Preparation for selection in next } \\
\text { step }\end{array}$ \\
\hline & $\begin{array}{l}\text { Iterate Feature Selection } \\
\text { (Group by PRIORITY } \\
\text { field) }\end{array}$ & $\begin{array}{l}\text { Separate IBAs into two layers } \\
\text { based on priority (Global/State) }\end{array}$ \\
\hline & Dissolve & $\begin{array}{l}\text { Remove any remaining } \\
\text { overlapping polygon areas }\end{array}$ \\
\hline & Add Field & $\begin{array}{l}\text { Add "IBA_\%Value\%_Weight" } \\
\text { field (\%Value\% replaced with } \\
\text { the iterator value Global or State) }\end{array}$ \\
\hline & Calculate Fields & $\begin{array}{l}\text { Field value }=1 \text { (because the area } \\
\text { is present). Value used in final } \\
\text { prioritization analysis }\end{array}$ \\
\hline \multirow{2}{*}{ 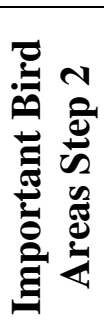 } & Iterate Feature Classes & $\begin{array}{l}\text { Select intermediate outputs } \\
\text { created in the user's workspace } \\
\text { geodatbase. }\end{array}$ \\
\hline & Delete & $\begin{array}{l}\text { Delete intermediate output } \\
\text { created by "Project" tool }\end{array}$ \\
\hline
\end{tabular}


The first step, "Important Bird Areas Step 1", created two feature classes from the original one feature class: one $\mathrm{f}$ containing globally important bird areas, and the other containing important bird areas for the state. A new column was also added to the two feature classes, called IBA_Global_Weight or IBA_State_Weight. This column, used as the weight in the wetland prioritization overlay analysis, was given the value 1 representing that the area was covered by a global or state 'Important Bird Area.' Step 2, "Important Bird Areas Step 2 (CleanUp)," deleted an intermediate file that was created in the first step.

\subsection{Critical Habitat Data Preparation and Automation}

The "Critical_Habitat_Polys" data layer from the National Wetlands Inventory (NWI) was originally provided by ecos.fws.gov, but was refined by the NWI to only show the critical habitat regions for endangered and threatened species that depend on wetlands or deep-water habitats for at least one part of their life cycle. Table 5-2 shows the complete workflow of the "Critical Habitat (Polygons) Data Prep" toolset. Five main models were created to complete the following five main steps. The model for Step 3 was customized using Python script tools.

Table 5-2. Data Preparation Steps for Critical Habitat Polygons Dataset

\begin{tabular}{|c|c|c|}
\hline & Operation Performed & Reasoning / Explanation \\
\hline \multirow{3}{*}{ 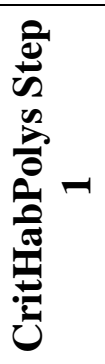 } & Clip & $\begin{array}{l}\text { Clips the Critical Habitat Polygon data to the } \\
\text { boundary of California }\end{array}$ \\
\hline & $\begin{array}{l}\text { Iterate Feature } \\
\text { Selection }\end{array}$ & $\begin{array}{l}\text { Select Endangered/Threatened species. The } \\
\text { following steps are performed for each iteration } \\
\text { (Endangered and Threatened) }\end{array}$ \\
\hline & Copy Features & $\begin{array}{l}\text { Create separate feature classes for endangered } \\
\text { species and for threatened species. }\end{array}$ \\
\hline \multirow{5}{*}{ 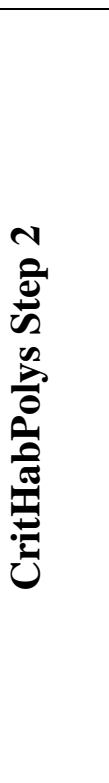 } & Make Feature Layer & $\begin{array}{l}\text { Convert threatened and endangered feature classes } \\
\text { to feature layers in preparation for following steps }\end{array}$ \\
\hline & Dissolve & $\begin{array}{l}\text { Dissolve threatened and endangered features based } \\
\text { on common name ("comname"). Creates two } \\
\text { intermediate outputs, one for endangered and one } \\
\text { for threatened species. }\end{array}$ \\
\hline & $\begin{array}{l}\text { Iterate Feature } \\
\text { Selection }\end{array}$ & $\begin{array}{l}\text { Iterate feature selection based on Object ID (OID) } \\
\text { in the newly created dissolve feature class. }\end{array}$ \\
\hline & Multipart to Singlepart & $\begin{array}{l}\text { Iterate through and create single-part feature } \\
\text { classes for each common name OID in each } \\
\text { intermediate data layer. } \\
\text { (Dissolve on OID used in conjunction with Iterate Feature Selection } \\
\text { to avoid trouble selecting features with apostrophes in the name) }\end{array}$ \\
\hline & Add Fields & $\begin{array}{l}\text { "i_Endangered_Weight" or } \\
\text { "i_Threatened_Weight" added to newly created } \\
\text { feature classes }\end{array}$ \\
\hline
\end{tabular}




\begin{tabular}{|c|c|c|}
\hline & Operation Performed & Reasoning / Explanation \\
\hline & Calculate Field & $\begin{array}{l}\text { Set the "i_Endangered_Weight" / } \\
\text { "“i_i_Threatened_Weight" fields equal to } 1.0\end{array}$ \\
\hline \multirow{5}{*}{ 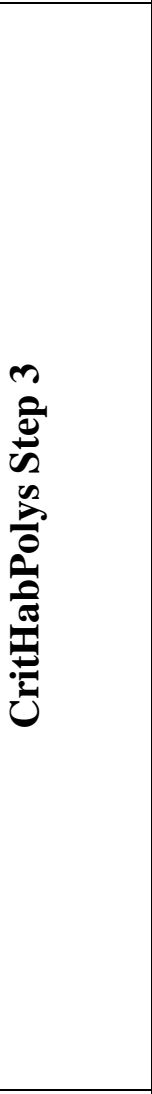 } & Union & $\begin{array}{l}\text { Find regions of overlap between different critical } \\
\text { habitat regions for the different endangered or } \\
\text { threatened species. Creates two feature classes. } \\
\text { One exhibiting all areas of overlap for threatened } \\
\text { species, and one exhibiting all areas of overlap for } \\
\text { endangered species. }\end{array}$ \\
\hline & Add Fields & $\begin{array}{l}\text { Fields added: (CHp_EndgSp_Count, } \\
\text { CHp_EndgSp_List, ES_CountStandardized) or } \\
\text { (CHp_ThrSp_Count, CHp_ThrSpeList, } \\
\text { TS_CountStandardized) }\end{array}$ \\
\hline & $\begin{array}{l}\text { Calculate Species List } \\
\text { (Custom Script Tool) }\end{array}$ & $\begin{array}{l}\text { Calculate list of species dependent on each unique } \\
\text { area of overlap. Output stored in field: } \\
\text { CHp_EndgSp_List/CHp_ThrSp_List }\end{array}$ \\
\hline & $\begin{array}{l}\text { Calculate } \\
\text { Endangered/Threatened } \\
\text { Species Count (Custom } \\
\text { Script Tool) } \\
\end{array}$ & $\begin{array}{l}\text { Calculate number of species dependent on each } \\
\text { unique area of overlap. Output stored in field: } \\
\text { CHp_EndgSp_Count/CHp_ThrSp_Count }\end{array}$ \\
\hline & $\begin{array}{l}\text { Standardize Weighting } \\
\text { Scores }\end{array}$ & $\begin{array}{l}\text { Standardize Endangered/Threatened Species count } \\
\text { into a score ranging from } 0-1 \text {, with } 1 \text { representing } \\
\text { the maximum number of species in the dataset that } \\
\text { are dependent on a single area. Output stored in } \\
\text { ES/TS Count_Standardized field. }\end{array}$ \\
\hline 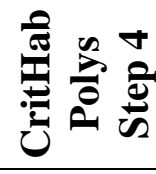 & $\begin{array}{l}\text { Feature Class to } \\
\text { Feature Class }\end{array}$ & $\begin{array}{l}\text { Delete unnecessary fields previously created by } \\
\text { the Union step, by using the Field Map parameter } \\
\text { in Feature Class to Feature Class. }\end{array}$ \\
\hline \multirow{2}{*}{ 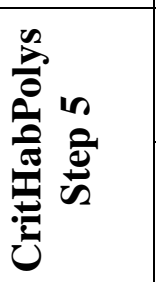 } & Iterate Feature Classes & $\begin{array}{l}\text { Select intermediate outputs created in the user's } \\
\text { workspace geodatabase. }\end{array}$ \\
\hline & Delete & $\begin{array}{l}\text { Delete all intermediate outputs created by data } \\
\text { preparation }\end{array}$ \\
\hline
\end{tabular}

The first main step after clipping the data to the boundary of California was to split up the different regions represented in the critical habitat feature class into separate feature classes according to their listing status (threatened or endangered), so that regions of different status could be weighted differently by the end-user. However, the critical habitat layer also had the added complexity that many different habitat ranges overlapped -not just threatened species habitat overlapping endangered species habitat, but one endangered species' habitat overlapping another. Because an area important to three endangered species could be argued to be much more important than an area important to only one endangered species, it was desirable to find a way to incorporate this extra level 
of information into the analysis. Figure 5-2 gives an example of this, with three overlapping circle representing species ranges. It is helpful to be able to see the areas where species ranges overlap, so as to determine areas that are most important to the most number of species.
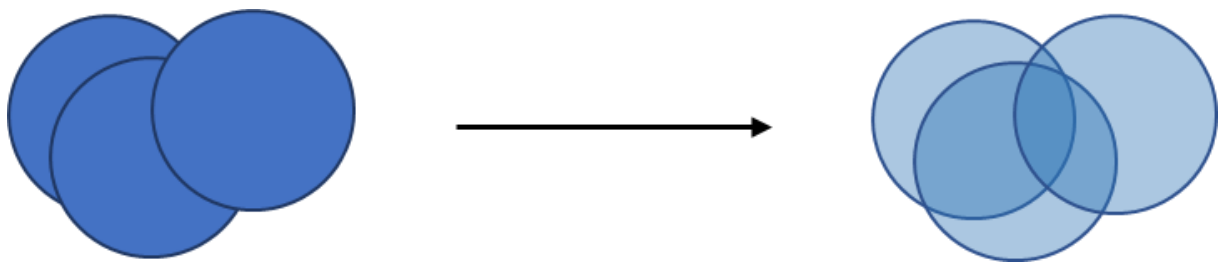

Figure 5-2: Incorporating Overlapping Critical Habitat Areas for Individual Species

To incorporate areas of overlap of different species ranges into the final dataset, Step 2 temporarily separated the feature classes for endangered / threatened species into individual layers for each unique species based on the species common name, and Step 3 merged those individual layers back together with the Union tool to create an output layer where each unique area of overlap of critical habitat for different species was in its own feature in the feature class. Figure 5-3 provides a conceptual diagram of this process.

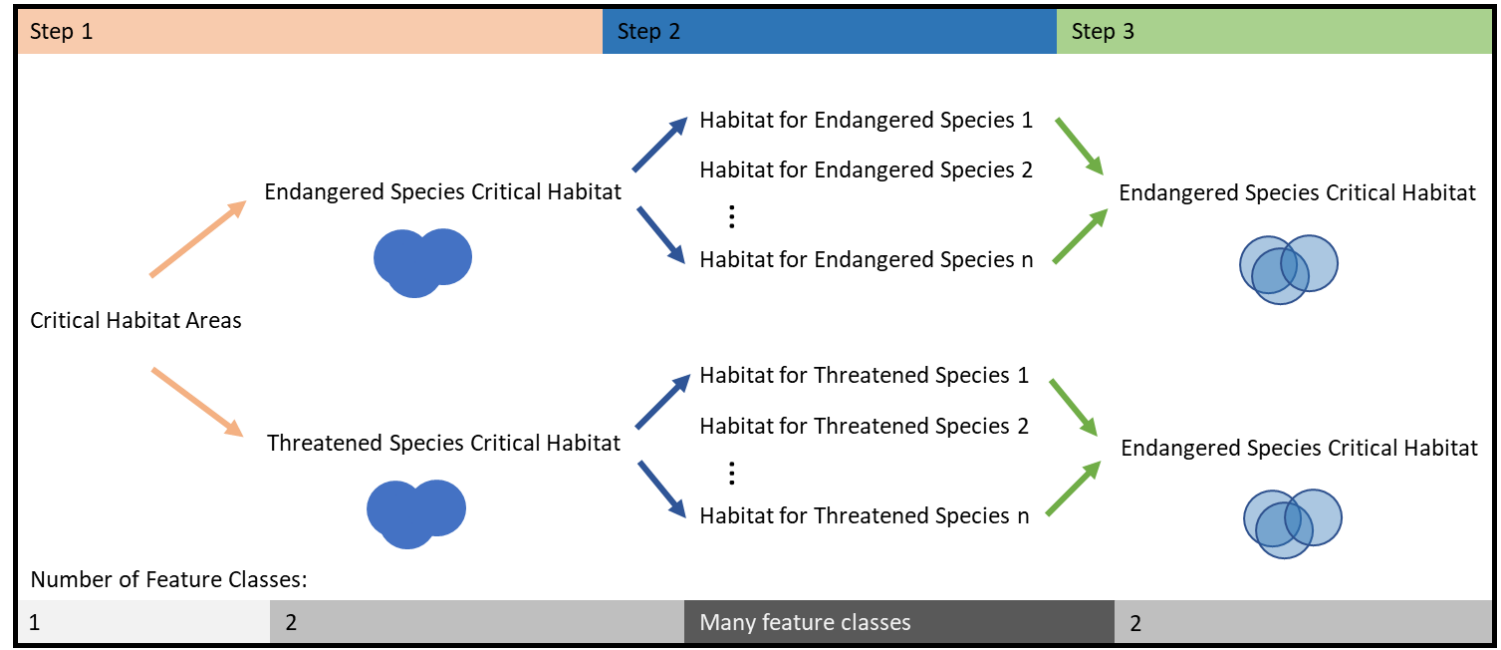

Figure 5-3: Concept Diagram for Steps 1-3

It should be noted that as part of Step 2, each species feature class was also given a new column in the attribute table ("i_Endangered_Weight" or "“i_Threatened_Weight") depending on whether the species was threatened or endangered, and the field was calculated as 1 . This then prepared the way to calculate the number of species that depended on any given area of overlap in Step 3, using a custom Python script that added all values in any column whose name began with "i_Endangered_Weight" or "i_Threatened_Weight". A similar function was performed with another custom Python script that concatenated all species names together, to create one concise list of species dependent on any given area. Table 5-3 shows a simplified example of what the important columns in the attribute table of the derivative feature class for threatened 
species would look like, if there were only two threatened species, and if their ranges partially overlapped.

Table 5-3. Example Attribute Table After Union for Two Unique Species Ranges

\begin{tabular}{|l|l|l|l|l|l|l|}
\hline OID & i_Threatened_Weight & comname & i_Threatened_Weight_1 & comname_1 & ThrSp_Count & Thr_Sp_List \\
\hline 1 & 1 & Delta Smelt & 0 & Null & 1 & Delta Smelt \\
\hline 2 & 0 & Null & 1 & Yosemite Toad & 1 & Yosemite Toad \\
\hline 3 & 1 & $\begin{array}{l}\text { Delta } \\
\text { Smelt }\end{array}$ & 1 & Yosemite Toad & 2 & $\begin{array}{l}\text { Delta Smelt, } \\
\text { Yosemite Toad }\end{array}$ \\
\hline
\end{tabular}

In Step 4, unnecessary columns created from the Union tool were deleted, and a custom index score was calculated for each feature, ranging from 0 to 1 , to quantify the number of endangered species in each area in a standardized way. For this index score, the number of species dependent on the area was divided by the maximum number of species in any given area in the dataset, such that the critical habitat area with the highest number of species dependent on it was calculated as 1 and everything else had a correspondingly lower score between 0 and 1 . The rest of the values were given a corresponding number between 0 and 1 based on number of endangered species. Figure 5-4 shows an example of how species count was converted to a standardized score for each feature in the feature class.
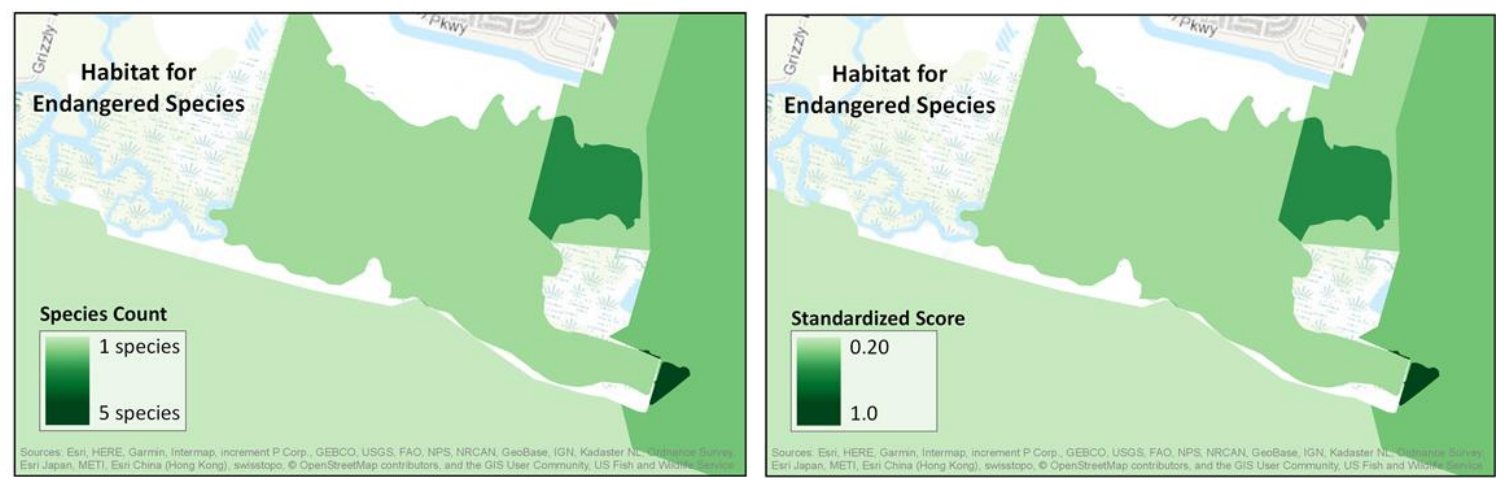

Figure 5-4: Critical Habitat Symbolized by Species Count and Standardized Score

\subsection{Critical Habitat Lines Data Preparation and Automation}

In addition to the critical habitat polygons provided by the NWI, critical habitat lines were also provided. This data layer consists of lines representing critical river habitats for two species: chinook salmon and steelhead trout. Four models were developed in the "Critical Habitat (Polygons) Data Prep" toolset to implement the complete workflow as shown in Table 5-4. 
Table 5-4. Data Preparation Steps for Critical Habitat Lines (Rivers)

\begin{tabular}{|c|c|c|}
\hline & Operation Performed & Reasoning / Explanation \\
\hline \multirow{7}{*}{ 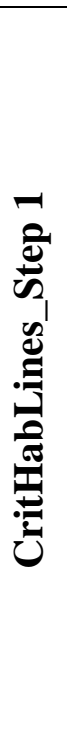 } & Clip & Clip river habitat file to $\mathrm{CA}$ boundaries \\
\hline & Pairwise Buffer & $\begin{array}{l}\text { Create a buffer of } 500 \text { meters (or whatever } \\
\text { distance the user specifies) around river lines }\end{array}$ \\
\hline & Dissolve & $\begin{array}{l}\text { Dissolve features based on common name } \\
\text { ("comname"). Creates intermediate output. }\end{array}$ \\
\hline & Add Fields & $\begin{array}{l}\text { "CritHabRiverine_Weight" field created in the } \\
\text { intermediate dissolve output. }\end{array}$ \\
\hline & Calculate Fields & $\begin{array}{l}\text { "CritHabRiverine_Weight" field calculated to } \\
\text { equal } 1\end{array}$ \\
\hline & Iterate Feature Selection & $\begin{array}{l}\text { Select feature iteratively by OID in the } \\
\text { intermediate dissolve output. }\end{array}$ \\
\hline & Multipart to Singlepart (x2) & $\begin{array}{l}\text { Create singlepart feature classes for each OID in } \\
\text { the intermediate dissolve output }\end{array}$ \\
\hline \multirow{4}{*}{ 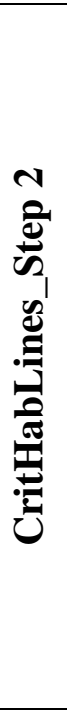 } & Union & $\begin{array}{l}\text { Find regions of overlap between different critical } \\
\text { habitat regions for the different species. Creates } \\
\text { intermediate output. }\end{array}$ \\
\hline & Add Fields (x2) & $\begin{array}{l}\text { Add fields to intermediate data layer that was } \\
\text { created via Union ("CHL_RivSp_Count" and } \\
\text { "Riv_CountStandardized") }\end{array}$ \\
\hline & $\begin{array}{l}\text { Calculate Critical Habitat } \\
\text { Riverine Species Count }\end{array}$ & $\begin{array}{l}\text { Calculate number of species dependent on each } \\
\text { unique area of overlap in the intermediate union } \\
\text { output. Output stored in "CHL_RivSp_Count" } \\
\text { field. }\end{array}$ \\
\hline & Calculate Species List & $\begin{array}{l}\text { Calculate list of species dependent on each } \\
\text { unique area of overlap in the intermediate union } \\
\text { output. Output stored in } \\
\text { "Riv_CountStandardized" field. }\end{array}$ \\
\hline \multirow{2}{*}{ 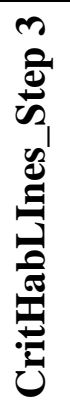 } & $\begin{array}{l}\text { Standardize Weighting } \\
\text { Score }\end{array}$ & $\begin{array}{l}\text { Standardize CHL_RivSp_Count into a score } \\
\text { ranging from } 0-1 \text {, with } 1 \text { representing the } \\
\text { maximum number of species in the dataset that } \\
\text { are dependent on a single area ( } 2 \text { species). } \\
\text { Output stored in "Riv_CountStandardized" field. }\end{array}$ \\
\hline & $\begin{array}{l}\text { Feature Class to Feature } \\
\text { Class }\end{array}$ & $\begin{array}{l}\text { Delete of unnecessary fields previously created } \\
\text { by the Union step, by using the Field Map } \\
\text { parameter in Feature Class to Feature Class. }\end{array}$ \\
\hline
\end{tabular}




\begin{tabular}{|c|c|c|}
\hline & Operation Performed & Reasoning / Explanation \\
\hline \multirow{2}{*}{ 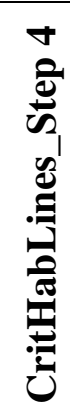 } & Iterate Feature Classes & $\begin{array}{l}\text { Select intermediate outputs created in the user's } \\
\text { workspace geodatabase. }\end{array}$ \\
\hline & Delete & $\begin{array}{l}\text { Delete intermediate output created by data } \\
\text { preparation }\end{array}$ \\
\hline
\end{tabular}

The first step in the data preparation for this layer was to create a 500 meter buffer area around each river. Then the same approach used for the critical habitat polygons was used for the critical habitat lines, to find areas of overlap and compute how many species ( 1 or 2) were dependent on a given river area. The final index score for the river habitat layer was again standardized, so a value of 1 would mean both species depended on that river, while a value of 0.5 would mean only one species was dependent on that river area. Figure 5-5 displays two maps that shows the critical river habitat areas symbolized by species count and then by the standardized score.
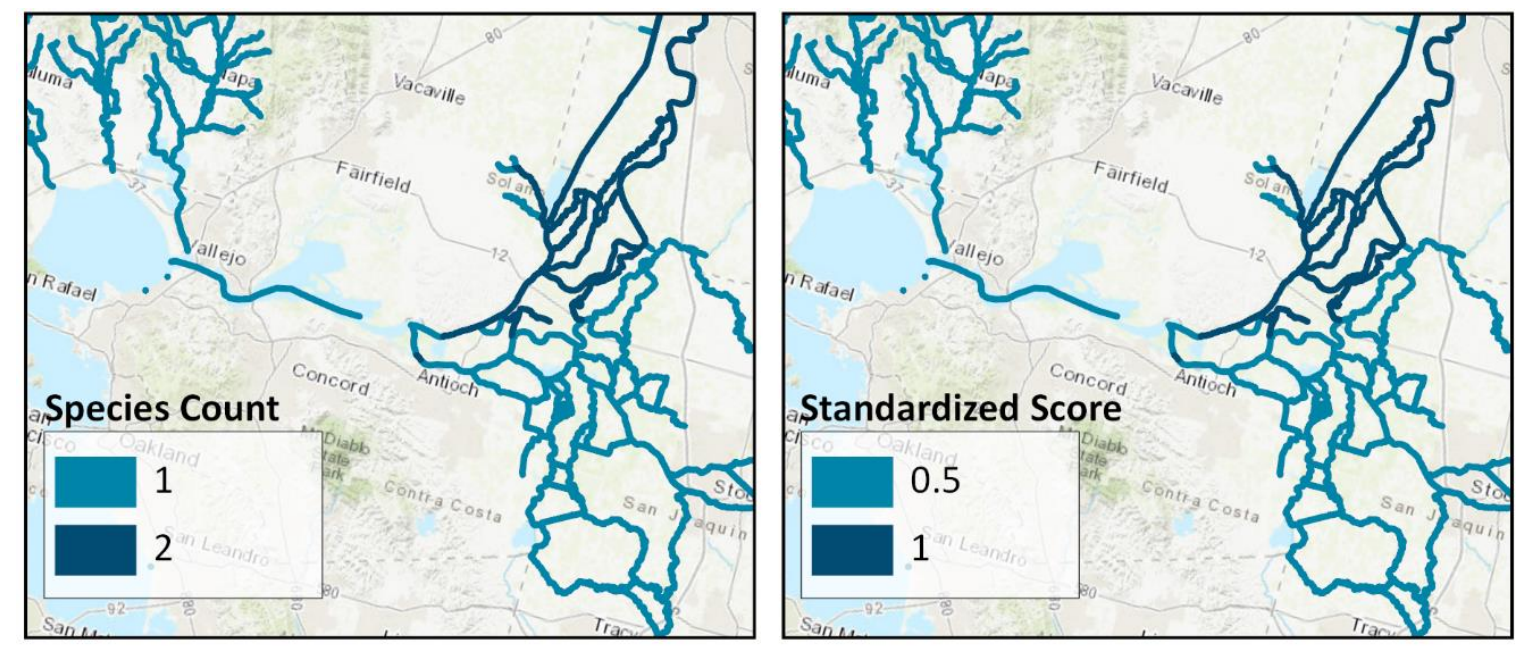

Sources: Esri, HERE, Garmin, Intermap, increment P Corp., GEBCO, USGS, FAO, NPS, NRCAN, GeoBase, IGN, Kadaster NL, Ordnance Survey, Esri Japan, METI, Esri China (Hong Kong), swisstopo, ( OpenStreetMap contributors, and the GIS User Community, US FWS

Figure 5-5: Count and Standardized Score Comparison for Critical River Habitat

\subsection{Wetland Data Preparation}

The wetland polygon feature layer from the USFWS was the basis for this analysis, but the original dataset includes 896,544 features and is about one gigabyte in size. It is also very detailed with over $153,000,000$ vertices. The large size of the wetland data dramatically extended the processing time for both data analysis and data loading in the web application. Because of these obstacles, it was necessary to decrease the size and detail of the wetland data without losing any critical information. A model, Prioritization Analysis Step 2, was created into the Wetland Prioritization Analysis toolset as an 
optional (but recommended) step before going forward with the final prioritization analysis (See again Figure 5-1).

Table 5-5 summarizes the main steps involved in this tool. To reduce the data size, it was decided that all wetlands within 500 meters of at least one prioritization factor would be kept, while the rest would be excluded from the analysis, since those wetlands do not meet any prioritization criteria. This was done in the first part of Prioritization Analysis Step 2.

Table 5-5. Wetland Data Preparation (Prioritization Analysis Step 2)

\begin{tabular}{|l|l|}
\hline Operation Performed & Reasoning / Explanation \\
\hline Make Feature Layer & Preparation for creating a selection in the next step \\
\hline Select Layer By Location & $\begin{array}{l}\text { Select all wetlands within } 500 \text { meters of other } \\
\text { important factors }\end{array}$ \\
\hline Aggregate Polygons & $\begin{array}{l}\text { Create a generalized outline around the wetland } \\
\text { areas. Parameters: } 500 \text { meters, 100 meters squared } \\
\left(\mathrm{m}^{2}\right), 100 \text { m² }\end{array}$ \\
\hline $\begin{array}{l}\text { Clip (Optional Step for Small } \\
\text { Servers, not included in } \\
\text { toolset model) }\end{array}$ & $\begin{array}{l}\text { Clip the aggregate polygons output to a study area to } \\
\text { be used in a geoprocessing service and web } \\
\text { application on a server with limited abilities }\end{array}$ \\
\hline
\end{tabular}

Figure 5-6 shows an example map of this, where pink represents wetlands that were eliminated because they fell outside of the 500-meter buffer area of all prioritization factors. Green represents wetlands that were kept because they were within 500-meters of the buffer area around river habitats (shown in purple). To enhance visualization of data in the web application, users would still be able to see a raster tile layer of all wetlands in the final web application. 


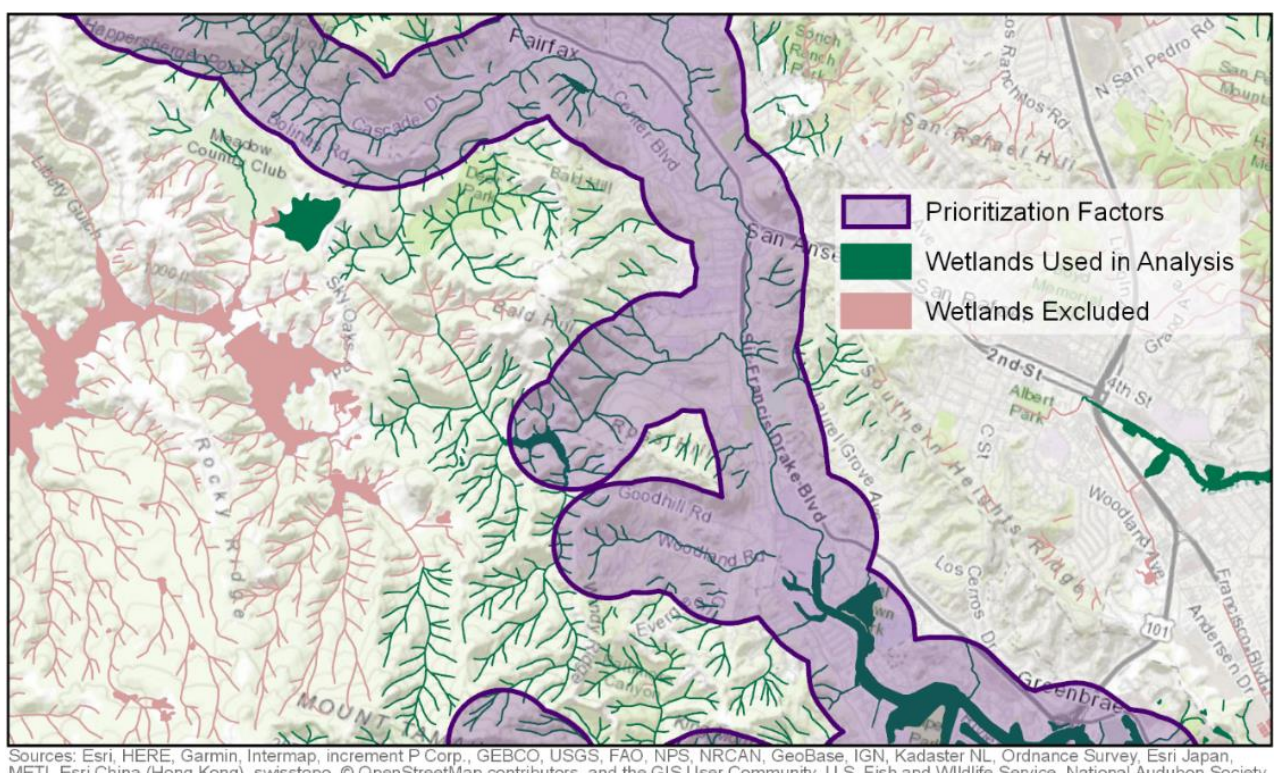

Figure 5-6: Only wetlands within 500 meters of prioritization criteria were kept

However, the wetlands data was still too large for web map display after deleting less relevant wetland features. This was mainly caused by the very detailed shapes of wetland polygons. To further decrease the number of vertices in the data, the "Aggregate Polygons" tool in ArcGIS Pro was used to create a more generalized outline of the wetland features. Figure 5-7 displays an example of the original wetland areas overlaid on top of the broader aggregated wetland areas that were created.

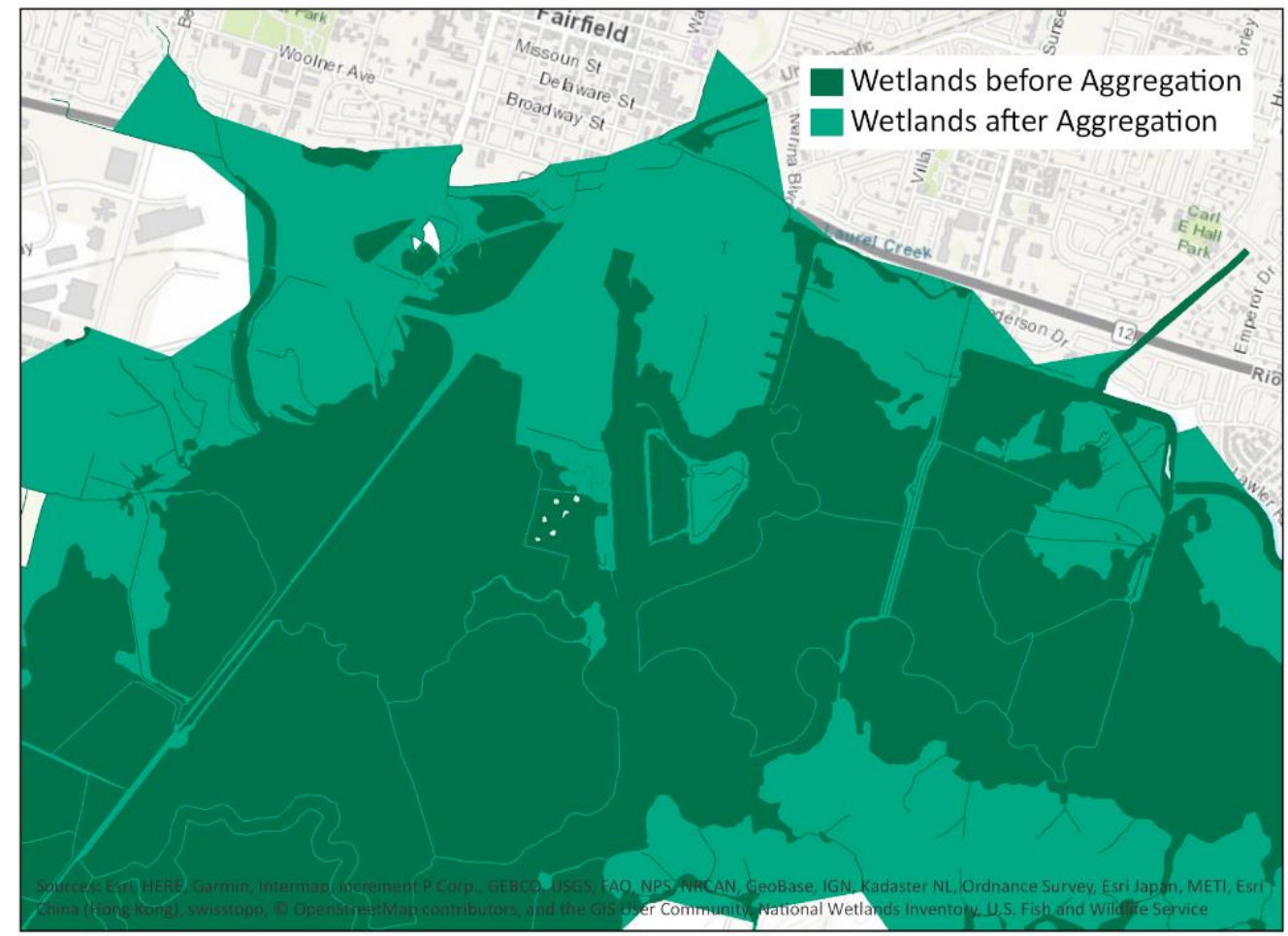

Figure 5-7: Original Wetland Area Compared Against Simplified Outline 
Due to the limited power of the hosting server in this pilot project, the distance and area parameters for this tool were set at 500 meters for the aggregation distance, 100 meters squared (m2) for the minimum area, and $100 \mathrm{~m} 2$ for the minimum hole size. With these settings, the wetland layer can be properly displayed on the web application for demonstration purpose. However, the option to change the parameter values was integrated into the final Wetland Prioritization Analysis toolset, so those parameter values should be re-evaluated. For the tool parameters, the "aggregation distance" is the threshold distance used to define polygons that are to be aggregated together into one generalized shape. Features greater than the specified distance apart are not aggregated together. The "minimum area" parameter represents the minimum area kept in the final output. If a feature or aggregation of features is below this minimum area threshold, those features are excluded from the final output layer. The "minimum hole size" means that any hole within a feature class or aggregated feature class that falls below the minimum hole area will be filled-in for the final feature in the output layer.

While the "Aggregate Polygons" increased the total wetland area in the dataset, it led to a $93 \%$ reduction in the count of vertices in the data file which enabled the output of the final wetland prioritization analysis to display properly in a web map. The resulting wetland area approximation was used to clip the other factors in the analysis, resulting in the light green layer shown in Figure 5-8 that represent the "wetland" layer used in the final prioritization analysis (discussed further in section 5.6).

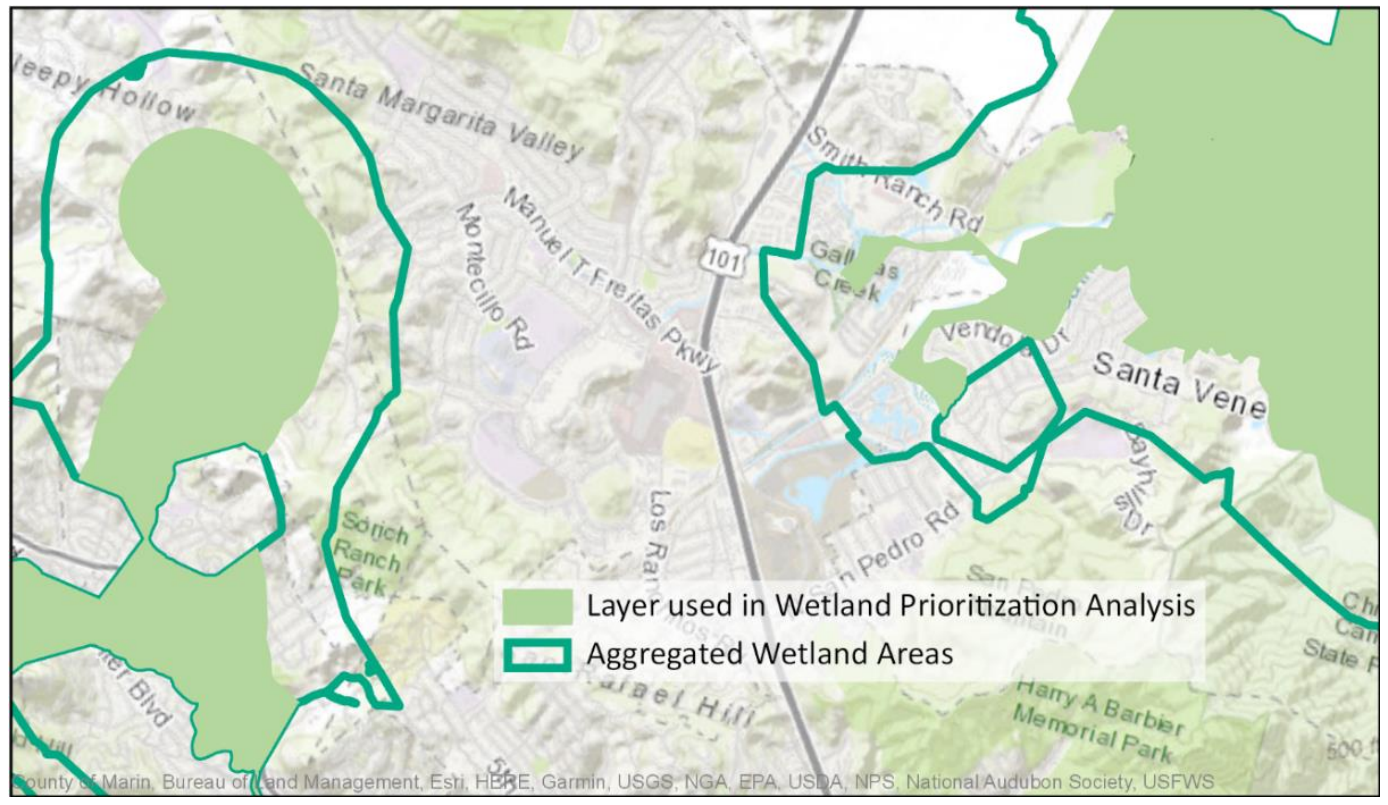

Figure 5-8: Aggregated Wetlands compared to Final Wetland Output for Prioritization Analysis

See Section 6.3.2 in Chapter 6 of this report for a further discussion on parameter values the Aggregate Wetlands tool, and for a discussion on suggested aggregation parameter values for future prioritization analyses.

As a side note, in addition to running the Aggregate Polygons tool, due to limited server capabilities of the server available at the University of Redlands, the data used for 
the geoprocessing service that was created in this project was further clipped to a study area near the San Francisco Bay called "San Pablo Bay Area" (Figure 5-9).

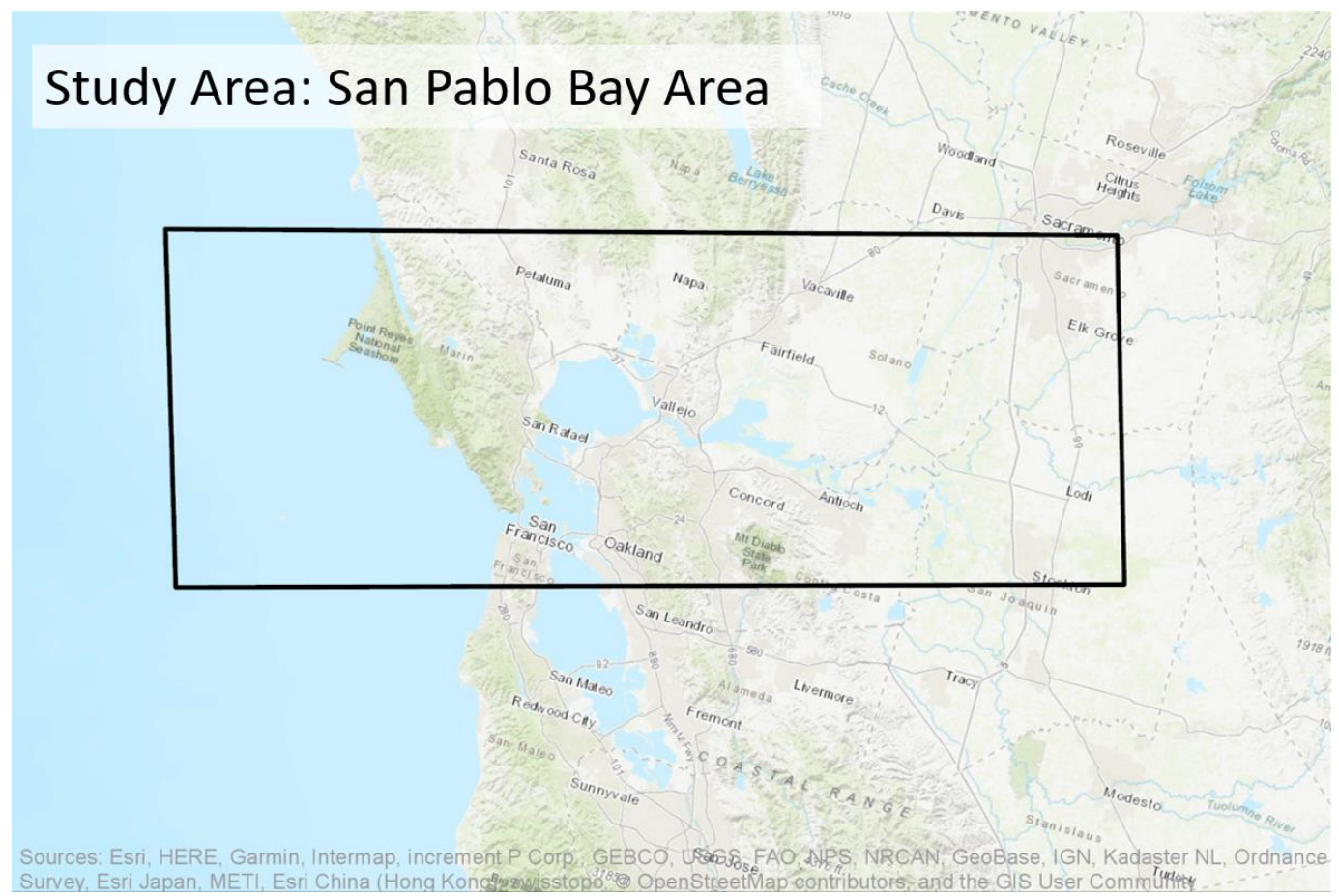

Figure 5-9: San Pablo Bay Area

Once the geoprocessing service and web application is adopted by the USFWS, this step should not be necessary, given that the National Wetlands Inventory has access to a more robust server system. Because of that, this last clip process was not automated like the rest of the analysis.

\subsection{Wetland Prioritization Analysis}

After preparing all the data, the final feature classes were integrated into the wetland prioritization analysis. The final layers integrated into the overlay analysis were: Important Bird Areas for California, Globally Important Bird Areas, Critical Habitat for Endangered Species, Critical Habitat for Threatened Species, Critical River Habitat Areas and wetland areas in California. Besides the Wetland Data Prep model described in Section 5.5, three additional models were created to complete the wetland prioritization analysis (Table 5-6). 
Table 5-6. The Wetland Prioritization Analysis

\begin{tabular}{|c|c|c|}
\hline & Operation Performed & Reasoning / Explanation \\
\hline \multirow[t]{2}{*}{ के } & Union & $\begin{array}{l}\text { Find unique areas of overlap between Globally } \\
\text { Important Bird Areas, Critical Habitat for } \\
\text { Endangered Species, Critical Habitat for } \\
\text { Threatened Species, Critical River Habitat Lines, } \\
\text { and California Wetlands Data } \\
\text { Also, re-project the data into the user-specified } \\
\text { output coordinate system if the data is not } \\
\text { already in that coordinate system }\end{array}$ \\
\hline & Add Fields & $\begin{array}{l}\text { Final_Index_Score column added to attribute } \\
\text { table }\end{array}$ \\
\hline \multirow{3}{*}{$\frac{1}{\tilde{D}^{2}}$} & Make Feature Layer & $\begin{array}{l}\text { Preparation for creating a selection in the next } \\
\text { step }\end{array}$ \\
\hline & Select Layer by Location & $\begin{array}{l}\text { Selects all wetland features that are within } 500 \\
\text { meters of any prioritization factors }\end{array}$ \\
\hline & Aggregate Polygons & $\begin{array}{l}\text { Create a generalized outline around the wetland } \\
\text { areas. Parameters: } 500 \text { meters, } 100 \text { meters } \\
\text { squared }\left(\mathrm{m}^{2}\right), 100 \mathrm{~m}^{2}\end{array}$ \\
\hline \multirow{3}{*}{$\frac{m}{\sum_{0}^{2}}$} & Project & $\begin{array}{l}\text { Projects the wetland dataset into a user-defined } \\
\text { projection (should be the same projection that } \\
\text { was specified as the output coordinate system for } \\
\text { the prioritization factors in Step } 1\end{array}$ \\
\hline & Clip & $\begin{array}{l}\text { Clip Unioned Important Factors to Aggregate } \\
\text { Polygons outline of Wetlands (created in } \\
\text { Wetland Data Prep) }\end{array}$ \\
\hline & Multipart to Single Part & $\begin{array}{l}\text { Separate multipart feature into single-part } \\
\text { features }\end{array}$ \\
\hline \multirow{3}{*}{ 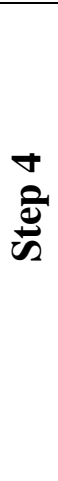 } & $\begin{array}{l}\text { Calculate Priority Score } \\
\text { (Custom Script Tool) }\end{array}$ & $\begin{array}{l}\text { Calculates priority index score based on user } \\
\text { defined weights and the individual weight fields } \\
\text { in the various input data. } \\
\text { Script also calculates the interval number to use } \\
\text { when the ScoreCategories are calculated. }\end{array}$ \\
\hline & $\underline{\text { Add Fields }}$ & $\begin{array}{l}\text { Add fields for cartographic visualization, } \\
\text { PriorityIndexScore_Rounded and } \\
\text { ScoreCategories }\end{array}$ \\
\hline & $\underline{\text { Calculate Fields }}$ & $\begin{array}{l}\text { Calculate PriorityIndexScore_Rounded and } \\
\text { ScoreCategories }\end{array}$ \\
\hline
\end{tabular}

In Step 1 of the Wetland Prioritization Analysis, all the prioritization factor layers were combined using the Union tool in ArcGIS Pro (Figure 5-10). 


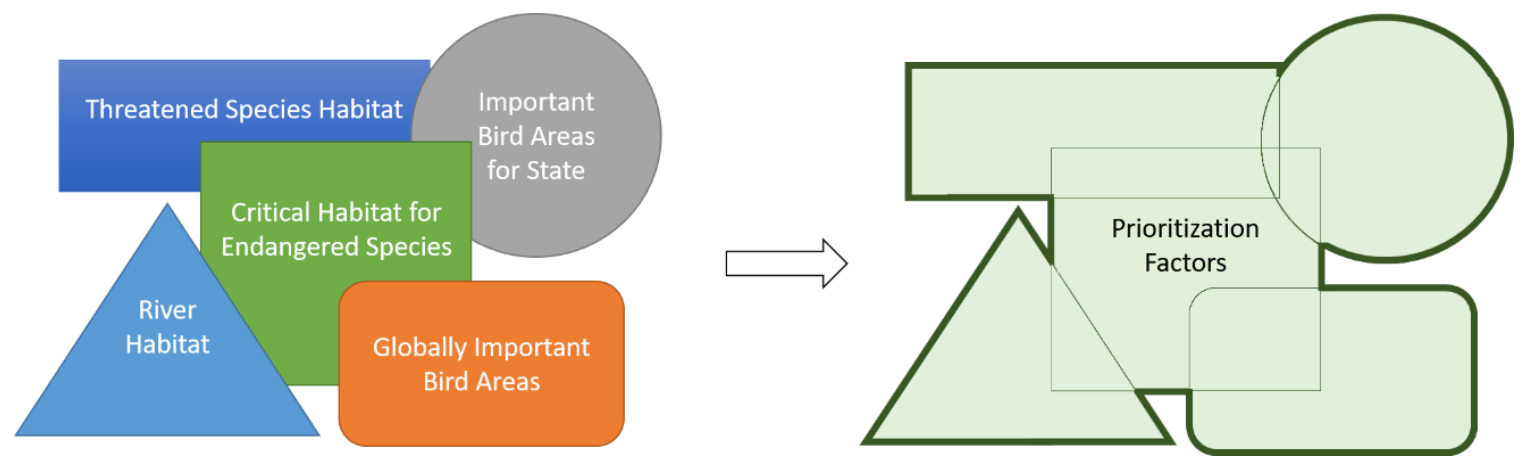

Figure 5-10: Prioritization Factors were combined into one dataset using "Union"

After that, a field was added that would eventually hold the final priority index score calculated by the end users of the tool (Table 5-6).

Table 5-6. Fields in Attribute Table of Union-ed Feature Class from Step 1

\begin{tabular}{|l|l|l|}
\hline \multicolumn{3}{|c|}{ Fields in Attribute Table } \\
\hline OBJECTID & TS_CountStandardized & RivSp_CountStandardized \\
\hline Shape & TS_List & RivSp_List \\
\hline Endangered_Species_Count & TS_Count & Final_Index_Score \\
\hline ES_CountStandardized & $\begin{array}{l}\text { Important_Bird } \\
\text { Area_Global_Wt }\end{array}$ & Shape_Length \\
\hline ES_List & IBA_State_Wt & Shape_Area \\
\hline Threathened_Species_Count & RivSp_Count & \\
\hline
\end{tabular}

Step 2 was an optional but recommended step for wetland data preparation as discussed previously in Section 5.5.

In Step 3, the output from Step 1 was clipped to the modified wetland data layer from Step 2 (or to the original wetlands data layer if that step was skipped). Multipart features were then split into single-part features.

A custom Python script was developed in Step 4 to calculate the final priority index score for wetland areas based on user-defined weights of importance for the various factors in the analysis. The formula used to calculate the custom index score for wetland areas was as follows:

$\{[$ (UserWeight x ImportantBirdAreas_State $)+($ UserWeight $x$ ImportantBirdAreas_Global $)+($ UserWeight $x$ EndangeredSpeciesScore $)+($ UserWeight $\mathrm{x}$ ThreatenedSpeciesScore) + (UserWeight x RiverHabitatScore)] / [Sum of UserWeight fields] \} x 100

To better visualize the priority levels of wetlands and display the priority index score, two new fields were then added. First, the priority index score is rounded from a double precision floating point number to integer and saved in a field called "PriorityIndexScore_Rounded." Second, a field named "ScoreCategories" was created into which priority index scores of all the wetlands in the feature class were sorted into 5 equal interval categories (Low, Medium-Low, Medium, Medium-High, High), for 
cartographic display purposes. This was done with the Calculate Fields tool with some custom Python scripting inside the Code Block (Figure 5-12).

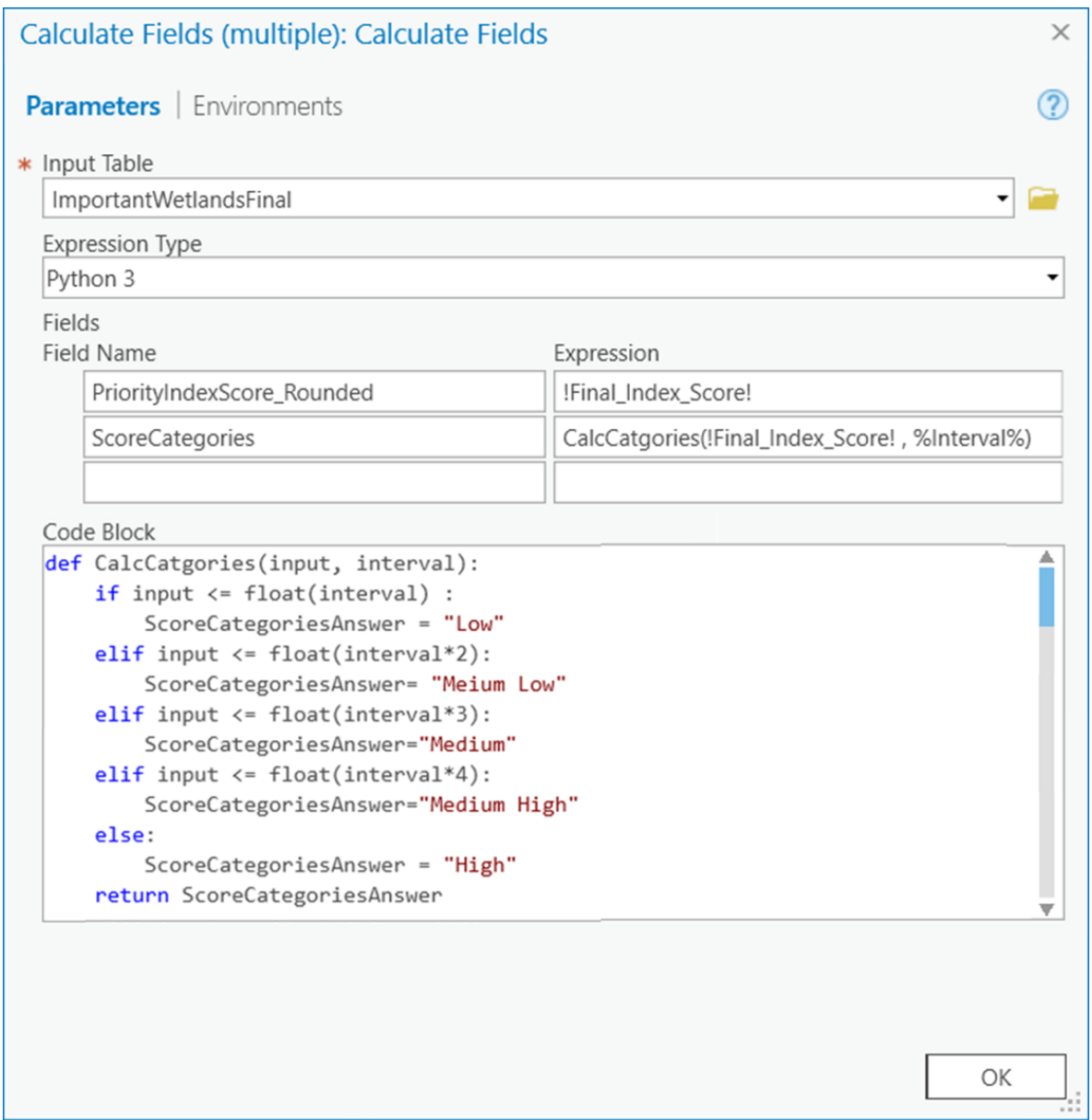

Figure 5-12: Calculate Fields Dialog Box in Wetland Prioritization Analysis Step 4

\subsection{Wetland Prioritization Geoprocessing Service}

After the creation of the four toolsets above, the last step (Step 4) in the "Wetland Prioritization Analysis" was converted into a geoprocessing service. Originally, the plan had been to publish a Web tool from ArcGIS Pro instead of a geoprocessing service from ArcMap, but due to complications with the server used by the University of Redlands, it was decided to publish the tool from ArcMap as a geoprocessing service instead.

To convert Step 4 from the Wetland Prioritization Toolbox into a geoprocessing service, the model was re-written as a custom script tool using arcpy, in order to avoid various difficulties that arose in publishing the geoprocessing service. Table 5-8 displays the various tasks that were included the final wetland prioritization script tool: 
Table 5-8. Wetland Prioritization Script Tool for Web

\begin{tabular}{|l|l|}
\hline \multicolumn{1}{|c|}{ Operation Performed } & \multicolumn{1}{|c|}{ Reasoning / Explanation } \\
\hline Copy Features & $\begin{array}{l}\text { Copies the input feature class into a new feature } \\
\text { class for the final output }\end{array}$ \\
\hline Calculate Priority Score & $\begin{array}{l}\text { Calculates priority index score based on user } \\
\text { defined weights and the individual weight fields } \\
\text { in the various input data. } \\
\{[\text { (UserW x IBA_S) }+(\text { UserW x IBA_G) }+(\text { UserW x } \\
\text { EngSpScore) }+(\text { UserW x ThrSpsore) }+(\text { UserW x } \\
\text { RiverHabScore)] / [Sum of UserW fields] } x \text { 100 }\end{array}$ \\
\hline Add Fields & $\begin{array}{l}\text { Add fields for cartographic visualization, } \\
\text { PriorityIndexScore_Rounded and } \\
\text { ScoreCategories }\end{array}$ \\
\hline Calculate Fields & $\begin{array}{l}\text { Calculate PriorityIndexScore_Rounded and } \\
\text { ScoreCategories }\end{array}$ \\
\hline
\end{tabular}

The script tool was then embedded inside a model in ArcMap (in a separate 10.5 version Toolbox) and run with the prepared wetland dataset that was clipped to a boundary around the San Pablo Bay region, which is near San Francisco. After running the model in ArcMap, the model and its associated data was published in ArcMap as a geoprocessing service. A copy and explanation of the script used in the script tool is provided in Appendix A, as well as a brief overview of a few of the parameter settings that were used to publish the service. After the geoprocessing service was published, it was integrated into a custom web application on the University of Redlands ArcGIS Enterprise server using Esri's Web AppBuilder. The final web application can be accessed at $<$ https://urspatial.redlands.edu/maps/apps/webappviewer/index.html?id=e6cf1bdf638c4a0c b11054a79f3451b2>.

Figure 5-13 shows the interface design of the web application, which includes both the map area on the right and input/output "Prioritize Wetlands" pane on the left.

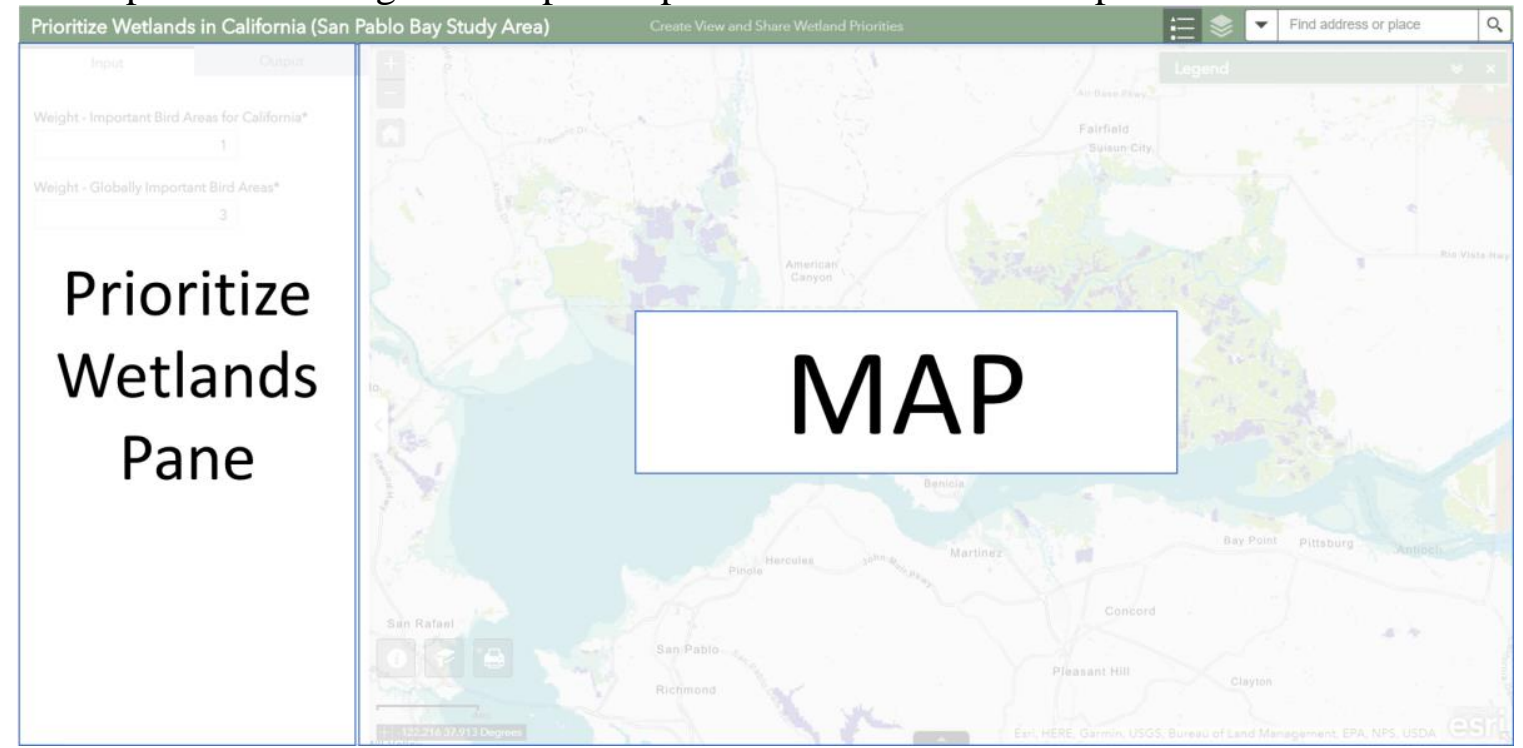

Figure 5-13: Opening Interface of Wetland Prioritization Web Application 
Users can explore wetlands types and visualize the analysis results in the map area. The web application also allows users to enter custom weights for various factors and output the final results in the different formats.

The creation of the web map was very standardized using Esri's Web AppBuilder, so the details of the creation of the web application will not be discussed for the most part. However, it is of interest to note that in the web application, streets and place name labels appear on top of the wetland layer that is generated (See Figure 5-14), even though the wetland layer created by the geoprocessing service normally goes to the top layer of the map automatically.

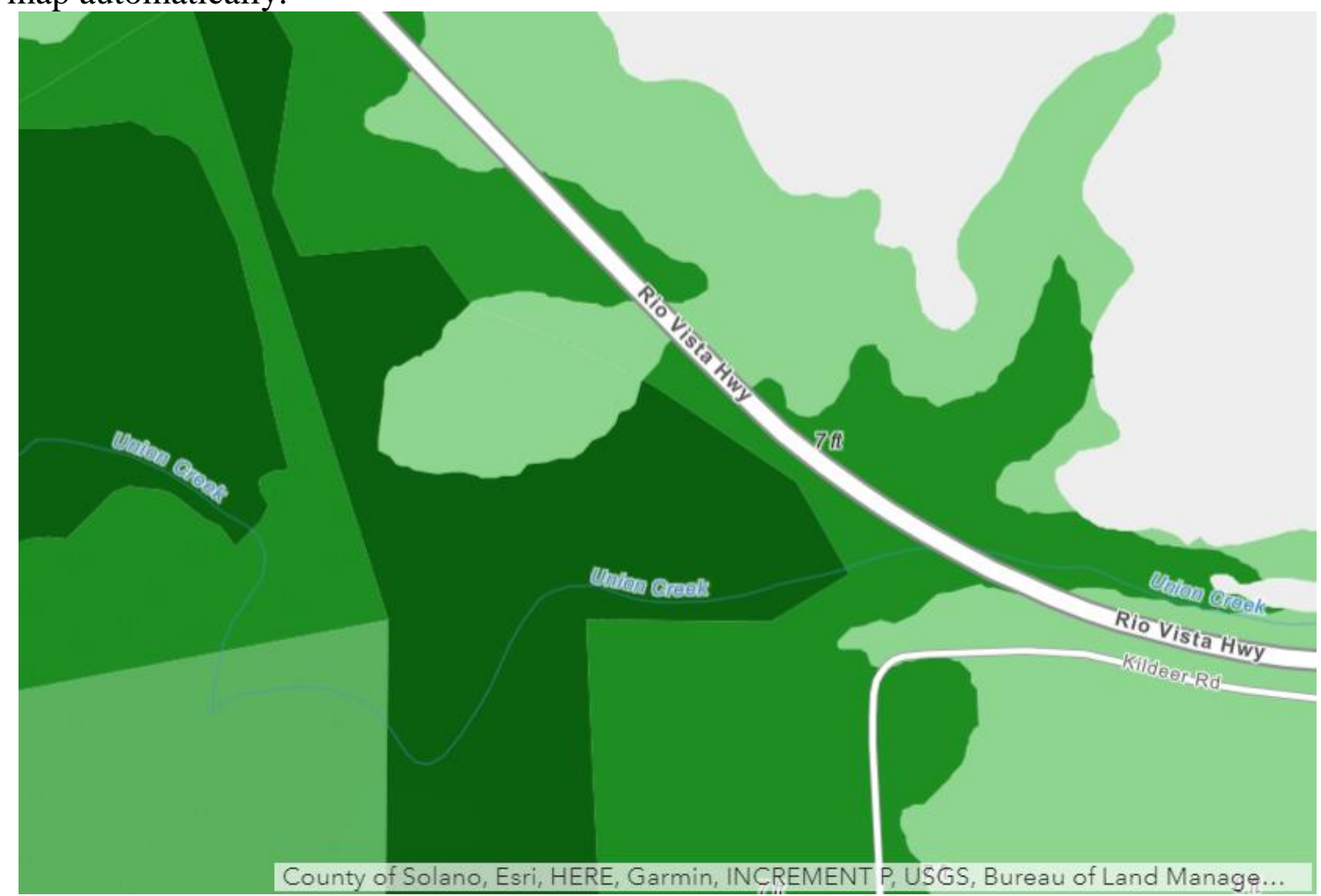

\section{Figure 5-14: Roads and Labels Appear on Top of Prioritized Wetlands for Reference}

To modify the map so that the reference data layers appeared on top of the prioritized wetland areas like this, Esri's "Vector Basemap Style Editor" was used to edit Esri's "World Terrain with Labels" basemap to exclude certain features such as background fill areas and various other distracting features. The edited layer was then added as a "Reference Layer" to the basemap in the web map that acted as the map for this web application, which meant that that vector layer would automatically display on top of whatever other data had been added to the map by the wetland prioritization geoprocessing service. The edited version of the vector basemap that acts as a reference layer for this map can be accessed at <https://univredlands.maps.arcgis.com/home/item.html?id=34795bfa087c406cbab2196b 8 cec01e2>.

Use of the web application will be detailed in Chapter 6. 


\subsection{Summary}

In conclusion, the first deliverable of this project was a ModelBuilder Toolbox with models and script tools (organized into toolsets), that enabled users to automate the wetland prioritization analysis, from the data preparation stage all the way to the final analysis. The second deliverable of this project was a custom web application with an embedded geoprocessing service to enable users to re-do the wetland prioritization analysis according to their own priorities. This next chapter will briefly discuss how this project will be used by employees of the U.S. Fish and Wildlife Service (USFWS), and the benefits provided to the client by this project. 


\section{Chapter 6 - Results and Analysis}

The final package of deliverables sent to the U.S. Fish and Wildlife Service included a geodatabase of all the original data collected, an ArcGIS Pro Toolbox containing the scripts for automating the prioritization analysis, a link to the final web application that was created, and an information packet explaining how to use the deliverables of the project and areas of the project in which further work should be done. This chapter will discuss how this project will be used and implemented by employees of the USFWS, and then will touch upon the main benefits of this project to the USFWS. It will then discuss additional work that should be completed before this project is used for decision making on a wide scale.

\subsection{Client Implementation and Use}

The two main deliverables of this project — an ArcGIS Pro Toolbox and a custom web application — will be used by two different audiences. The Wetland Prioritization Toolbox will be used by GIS employees in the USFWS who collaborate with the client (Cade London) to perform analyses for him. The toolbox will provide the most complete and robust form of the prioritization analysis. The web application created in this project provides a simplified version of the wetland prioritization analysis and will be used by the client and other decision makers to conduct wetland prioritization analyses online, without the use of desktop GIS software. Although it is recommended that any final decisions be based off of an ArcGIS Pro analysis (which will allow greater specificity in the analysis) the web application provides an easy way for stakeholders and decision makers to view and better understand wetland priorities, as well as to gather input from other stakeholders on their priorities.

In addition to being used to conduct wetland prioritization analyses for California, the ArcGIS Pro Wetland Prioritization Toolbox will also likely be used intermittently to update the online geoprocessing service as new data arises, as well as to perform the same prioritization analysis for states other than California. The toolbox may also be copied and modified so as to incorporate new data sources into the analysis. When using the toolbox, it is recommended that users create a new, empty geodatabase and call it something like "Wetland_Priorities_5June," or some other name that can easily show what the data are and which version it is (assuming the data are routinely updated). Users may save data to a different location if they wish, but they should note that all data that starts with "int_" (signifying intermediate data), is deleted in the last step of each toolset.

The web application created in this project, as stated before, will be used by the client and other decision makers to conduct the wetland prioritization analysis online. It will start out as a hosted service from the University of Redlands ArcGIS Enterprise server and will be used experimentally by Cade London and others of his associates to prioritize wetlands in California. Users of the web application will be able to use it without having any background knowledge of GIS.

Upon bringing up the web application in a browser and after closing a splash screen providing information on use of the web app, users are brought to the interface shown in Figure 6-1 where they can see a raster version of wetlands symbolized by type. 


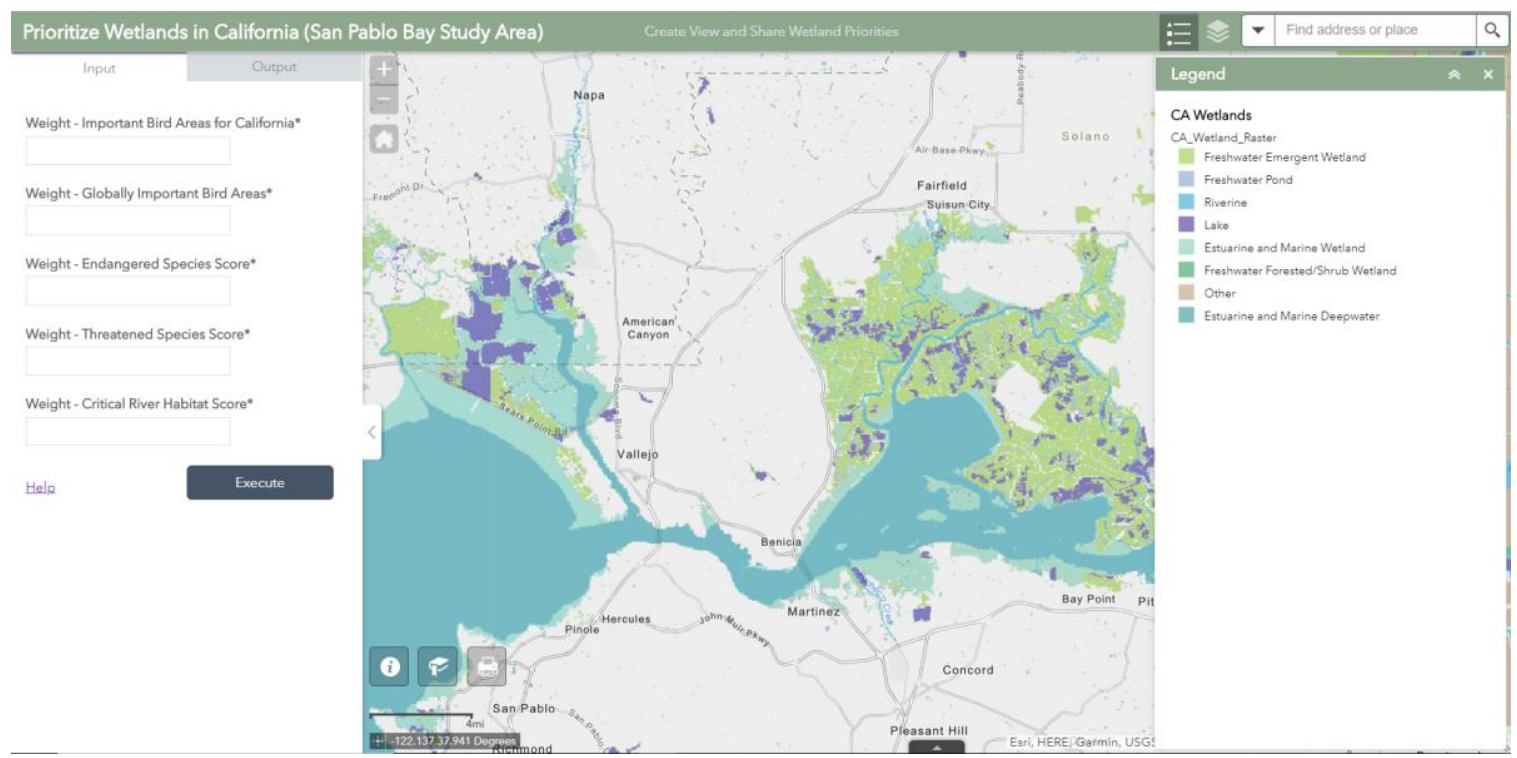

Figure 6-1: Opening Interface of Wetland Prioritization Web Application

There are eight different types of wetlands in the study area. The wetland types are not taken into account in the prioritization analysis but are provided as reference material. Figure 6-2 shows a close up of the legend for the original wetland layer and Figure 6-3 shows a close up of the map.

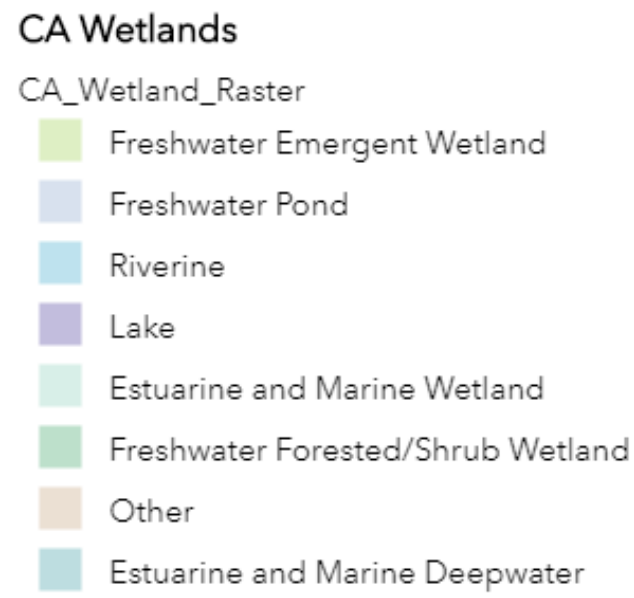

Figure 6-2 Legend for Opening Interface of Web Application 


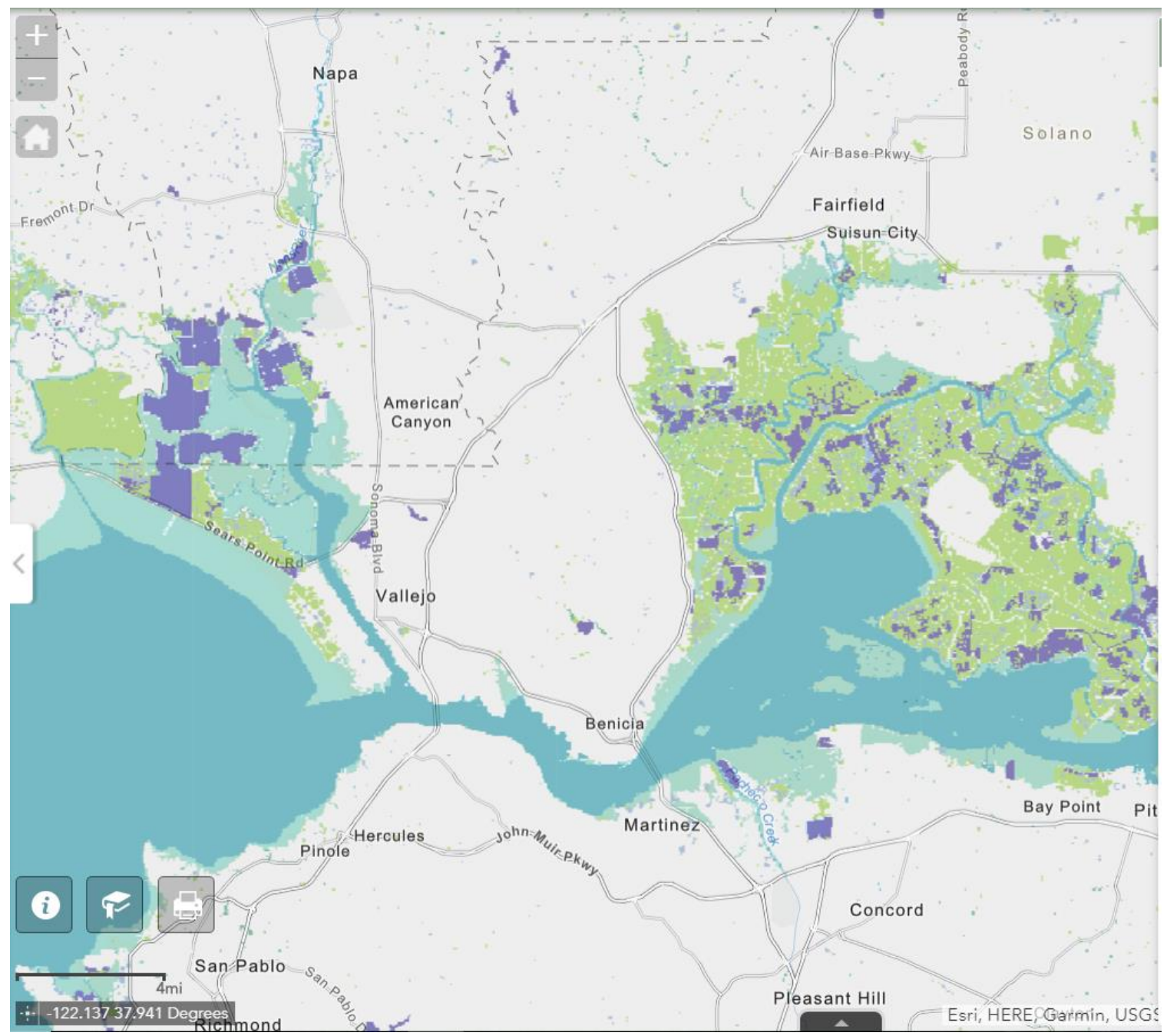

Figure 6-3: Map of Wetlands in the San Pablo Bay Area

To prioritize wetlands, users may enter their custom weights for the various factors in the analysis (Figure 6-4), and a resulting layer is generated displaying wetlands as prioritized by those custom weights (Figure 6-5). 


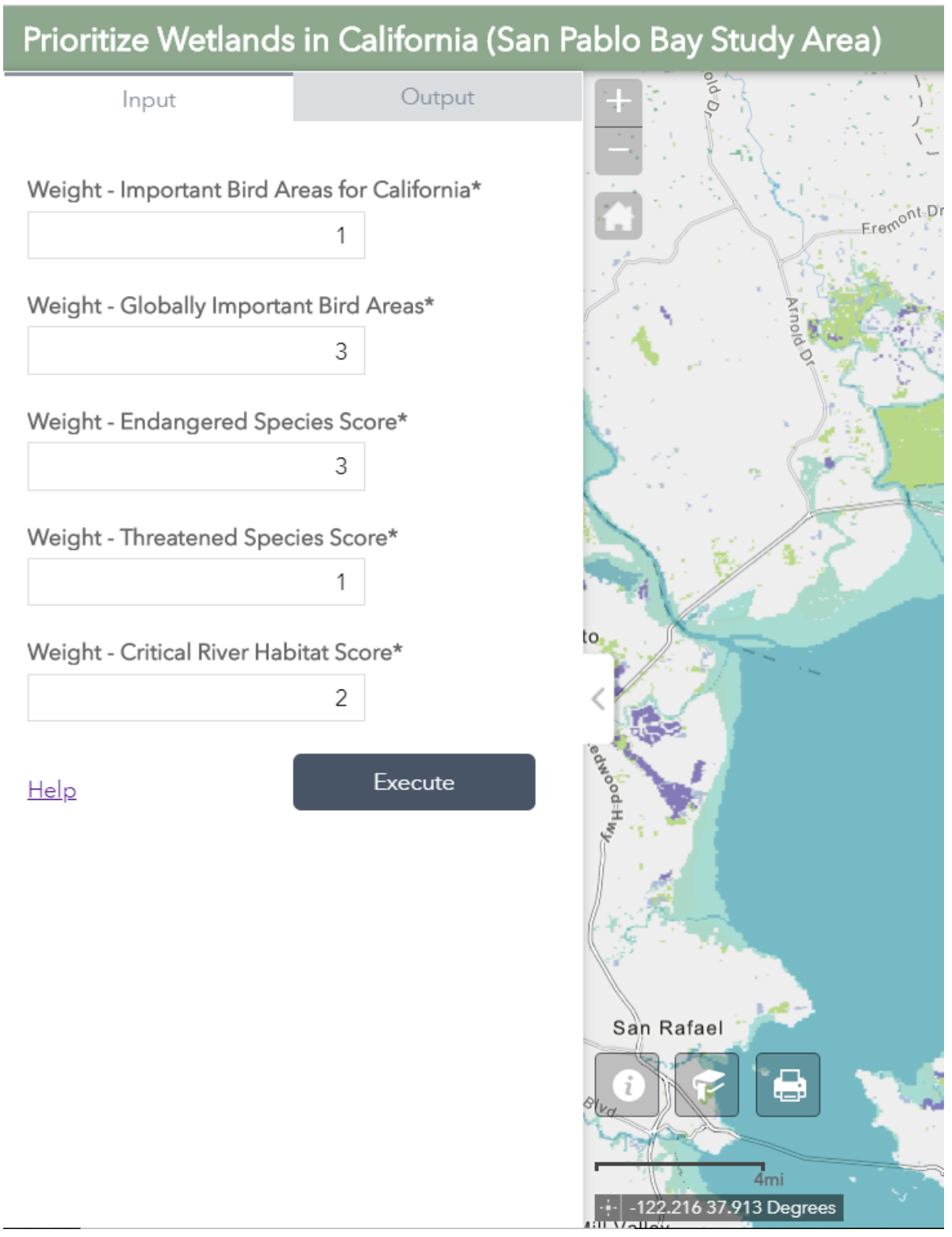

Figure 6-4: Prioritize Wetlands Pane with Custom Weights Entered 


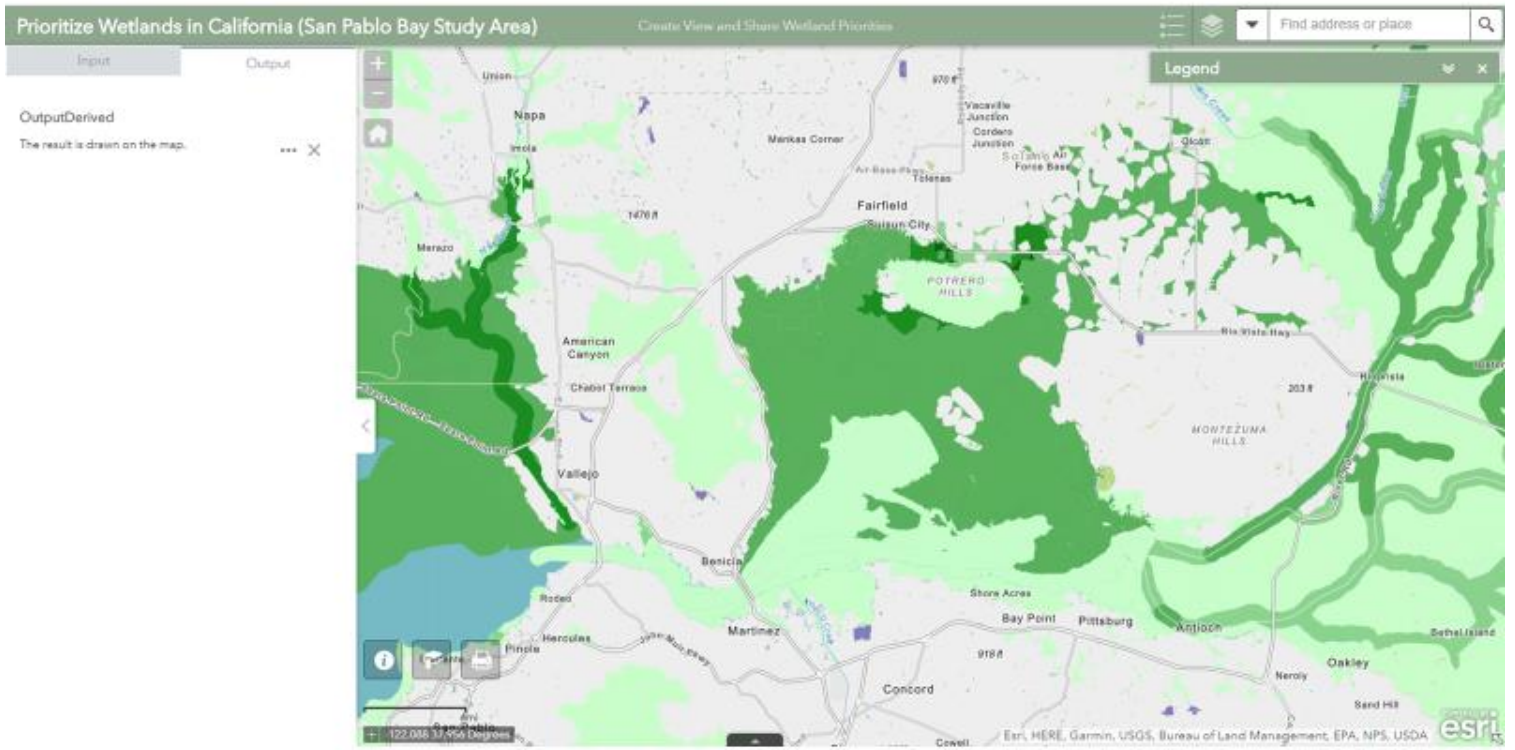

Figure 6-5: Output of a Wetland Prioritization Analysis in the California Wetland Prioritization Web Application

Figure 6-6 shows a close-up area of the output wetland priority map near Fairfield, California.

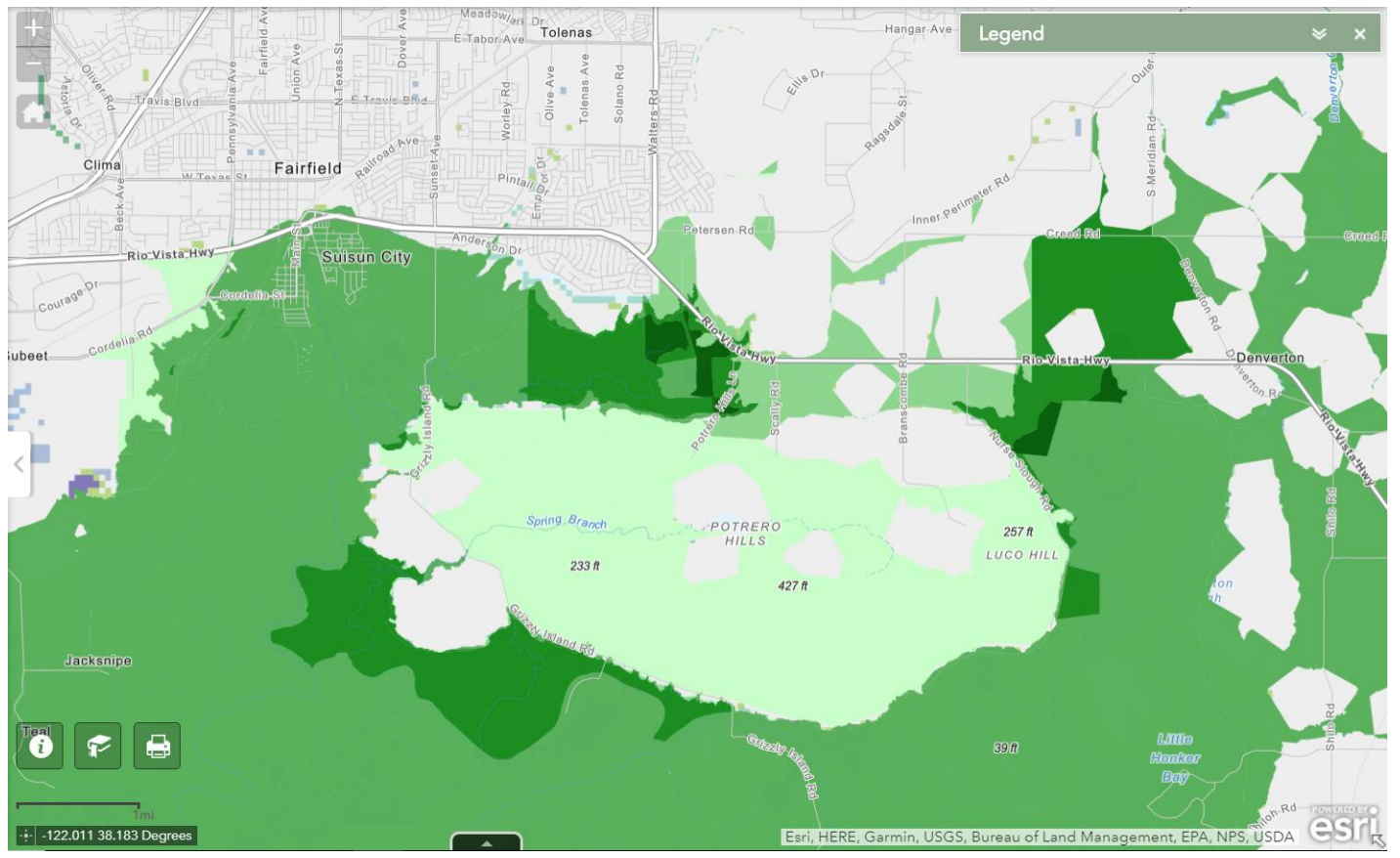

Figure 6-6: Close-up Map of Wetland Prioritized near Fairfield 
Users of the web application can then save images of the output map created by clicking the Print button shown in in the bottom left corner of the web application's map (Figure 6-7).

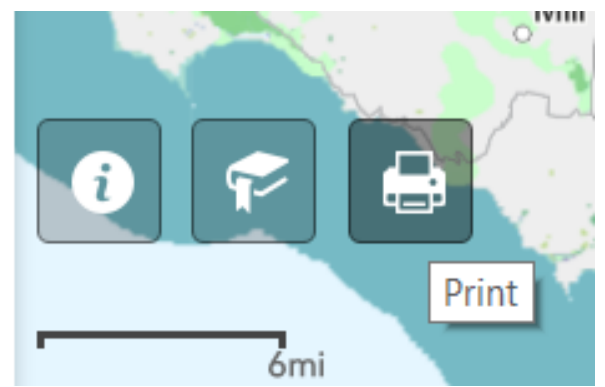

\section{Figure 6-7: Print Button in Web Application}

Users may also save the GIS version of the data layer created by clicking the three dots on the Output pane (Figure 6-8), and clicking Export to Feature Collection, which will then let users download the data in a format that can then be exported into a feature layer on ArcGIS Online.

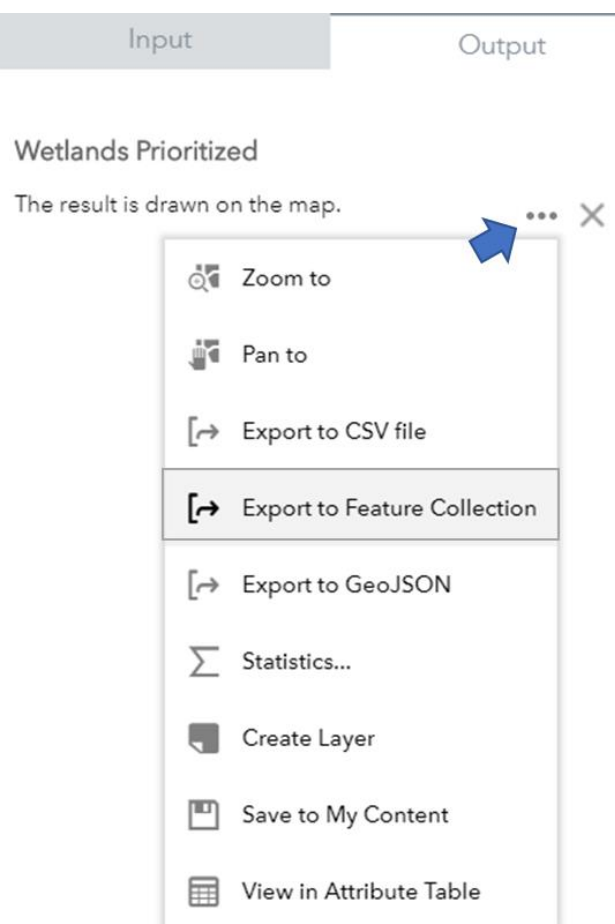

Figure 6-8: Saving the Output from the Web Application

In the future, GIS staff within the USFWS will re-publish the geoprocessing service onto USFWS servers, which are more robust than the server used in this project. After publishing the service, GIS professionals in the USFWS will re-create the web application onto USFWS servers, where the web analysis can be completed for a wider swath of California. 


\subsection{Benefits of This Project}

In automating the analysis process into one easy workflow, this project has immense amounts of potential value not only to employees in the USFWS, but for environmental and conservation managers and decision makers in general, as it enables scientists and other decision makers to quickly repeat analyses with different criteria and compare the results, without needing to deal with the background GIS work.

For example, Cade London can send the link to the web page to two different collaborators, one a biologist at a college out-of-state, and one a local land manager, and ask them to prioritize wetlands around the Travis Air Force Base (extent saved as a bookmark in the Wetland Prioritization Web Application), and to send him a copy of the resulting map (See Figure 6-9).

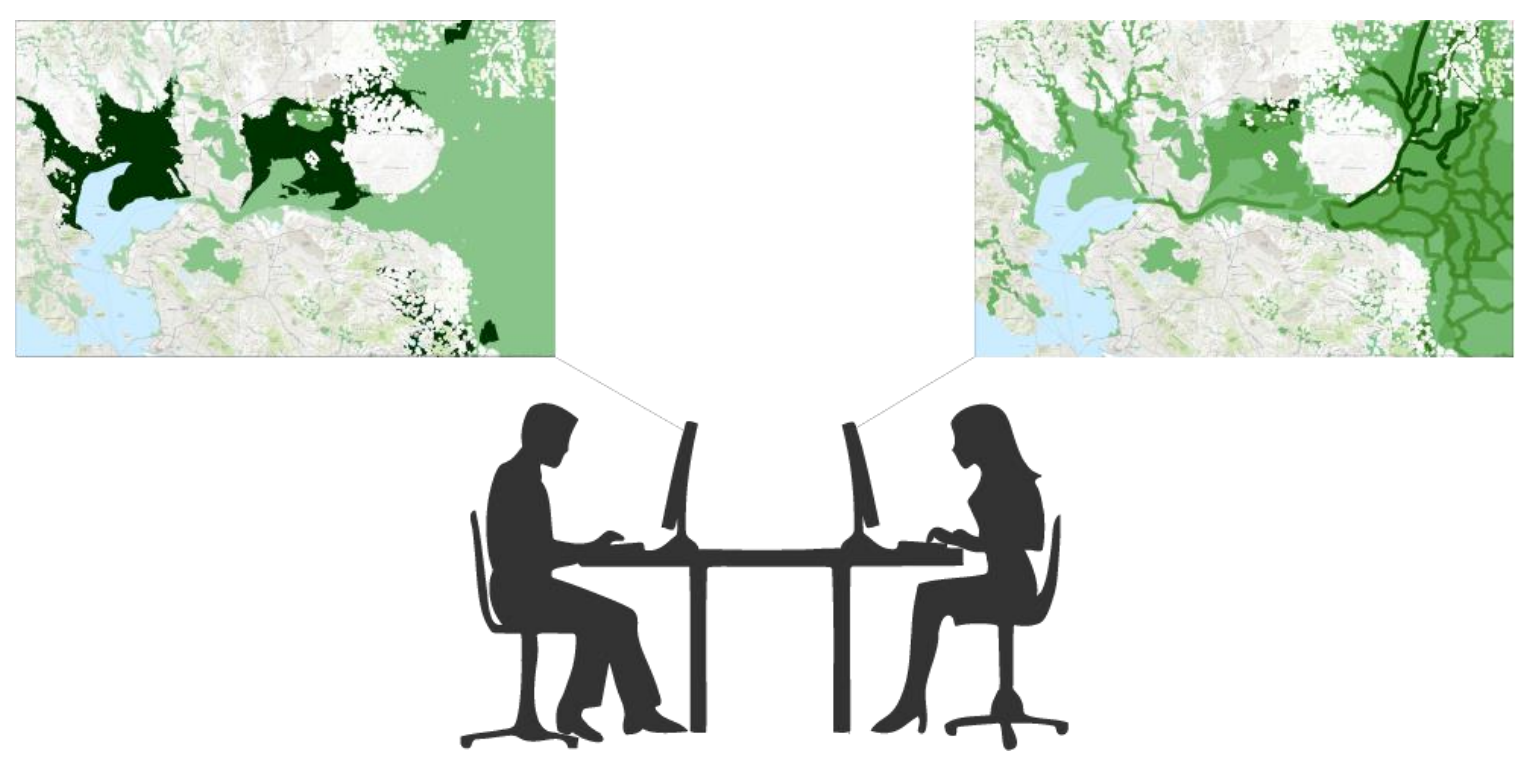

Figure 6-9: Wetland Prioritization in a Multi-Criteria Decision Analysis Framework

Alternatively, if one of the collaborators has access to ArcGIS Pro, they can use the wetland prioritization toolset instead of the web application, which simply gives them more options when generating a map of (and possibly a whole report on) their wetland prioritization results. The three can then meet over a conference call, discuss priorities, and find wetland areas of common interest where it would be most beneficial to focus efforts. This is an example of how, with the analysis process simplified into one tool, scientists and decision makers in the USFWS can now apply current and evolving scientific knowledge in a multiple criteria decision analysis framework in real-time.

\subsection{Discussion}

While this project provided a useful pilot analysis framework for prioritizing wetlands in California, the prioritization of wetlands, and of biological areas for conservation in general, is a widely studied and debated topic. Because of this, it is advised that additional work be performed, to ensure that the analysis tools created by this project generate scientifically robust suggestions. 


\subsubsection{Integration of More Input Data}

First, it is advisable for more input data to be integrated into the analysis, since limitations in time resulted in this pilot project only integrating a few data layers into the analysis (critical habitat for wetland-dependent endangered and threatened species, critical river habitat, and global and state important bird areas in California). Other influential factors that were considered by the client and his collaborators for integration into the future analysis include protected lands, USFWS lands, water quality data (if it could be found at a proper scale), climate projection data for future water distribution in California, suitability of the wetland for public access (wetland size and road access), and data from Esri's Green Infrastructure Initiative. It should also be noted that the USFWS Migratory Bird Data Center (https://migbirdapps.fws.gov/mbdc/databases/db_selection.html) has additional bird datasets including a "Bird Point Count Database" and the "North American Breeding Bird Survey" that were not used in this analysis, but that might prove beneficial to the analysis, particularly if combined with the Important Bird Area dataset from the National Audubon Society. In all, further research and discussions on what to integrate into the prioritization analysis are suggested in order to gain a broader perspective on priorities for both the USFWS, as well as other organizations and groups of people.

\subsubsection{Further Research on Buffer Distance and Area Thresholds}

A few empirical parameter settings were used in this project. For example, the river habitats layer was represented by the 500-meter buffer area along the rivers; and to eliminate the irrelevant wetlands, the wetlands dataset was clipped into the 500-meter buffer of the combined factor layer. Table 6-1 displays the current values for various buffer distances and areas that were used in the different steps of the analysis. While the choice of the 500-meter buffer distance was largely based on habitat connectivity ranges and buffer zones indicated in the literature review (see Section 2.3), the parameter settings in the "Aggregate Polygon" tool were mainly determined by the technological constraint in this project. It is recommended that further research on buffer distance and area thresholds be conducted for all of the empirical parameter settings in this project. The current empirical settings can be easily modified through changing the parameters in the models "CritHabLines Step 1" and "Prioritization Analysis Step 2" in the toolbox provided by the project, and the geoprocessing service can then be republished with the updated data. 
Table 6-1. Distance Areas and Thresholds in the Analysis

\begin{tabular}{|l|l|}
\hline \multicolumn{1}{|c|}{ Distance or Area Threshold } & \multicolumn{1}{c|}{$\begin{array}{c}\text { Value Used in } \\
\text { Analysis }\end{array}$} \\
\hline $\begin{array}{l}\text { Buffer distance around Critical River } \\
\text { Habitat Lines (CritHabLines_Step1) }\end{array}$ & 500 meters \\
\hline $\begin{array}{l}\text { Minimum distance from at least one other } \\
\text { factor in the analysis used when defining } \\
\text { which wetlands are integrated into the } \\
\text { analysis ("Search Distance" in } \\
\text { Prioritization Analysis Step 2) }\end{array}$ & 500 meters \\
\hline $\begin{array}{l}\text { Aggregate Polygons "Aggregation } \\
\text { Distance" (Prioritization Analysis Step 2) }\end{array}$ & 500 meters \\
\hline $\begin{array}{l}\text { Aggregate Polygons "Minimum Area" } \\
\text { (Prioritization Analysis Step 2) }\end{array}$ & $100 \mathrm{~m}^{2}$ \\
\hline $\begin{array}{l}\text { Aggregate Polygons "Minimum Hole } \\
\text { Size" (Prioritization Analysis Step 2) }\end{array}$ & $100 \mathrm{~m}^{2}$ \\
\hline
\end{tabular}

The Aggregate Polygons thresholds that were used in this project merit particular attention. As was mentioned earlier in this report, the second step in the wetland prioritization analysis ("Prioritization Analysis Step 2") allowed users to select wetlands within 500 meters of any other prioritization factors, and then perform the Aggregate Polygons tool on that data, to create a more simplified version of the wetland data layer. This tool created an output that essentially filled in small holes between wetlands and created a more generalized shape delimiting the wetland area (See Figure 6-10).

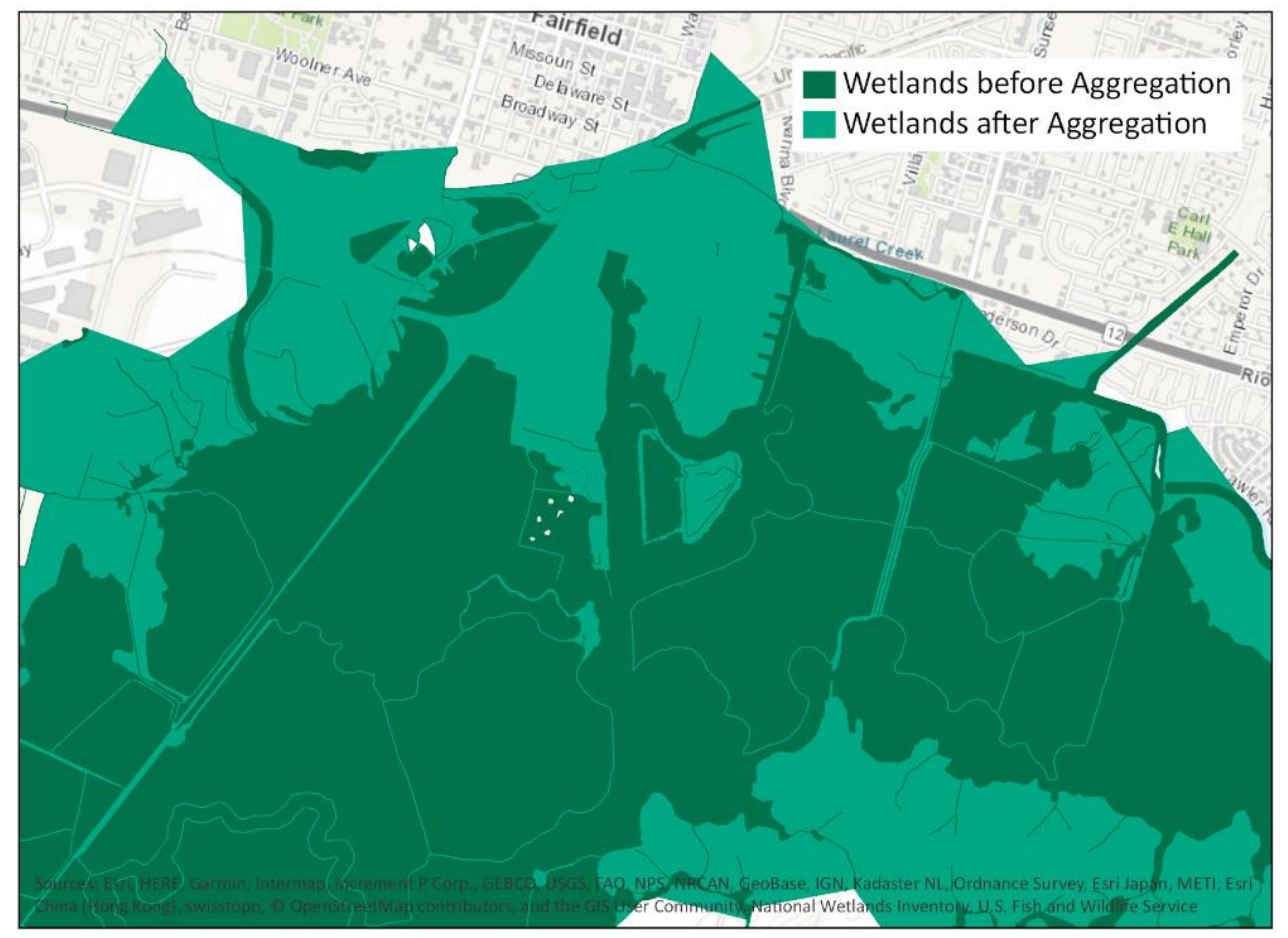

Figure 6-10: Example of Aggregated and Non-Aggregated Wetlands 
An analysis was conducted to compare eight threshold distances for the Aggregate Polygons tool, since it was desirable to find an appropriate threshold distance that made the data more manageable but that did not add too much area into the wetlands data. For a quick comparison, this analysis was conducted on the wetland data in a small region within the San Pablo Bay Area, called the "South of Fairfield" study area, which contains only about 12,000 features. Figure 6-11 shows the South of Fairfield study area.

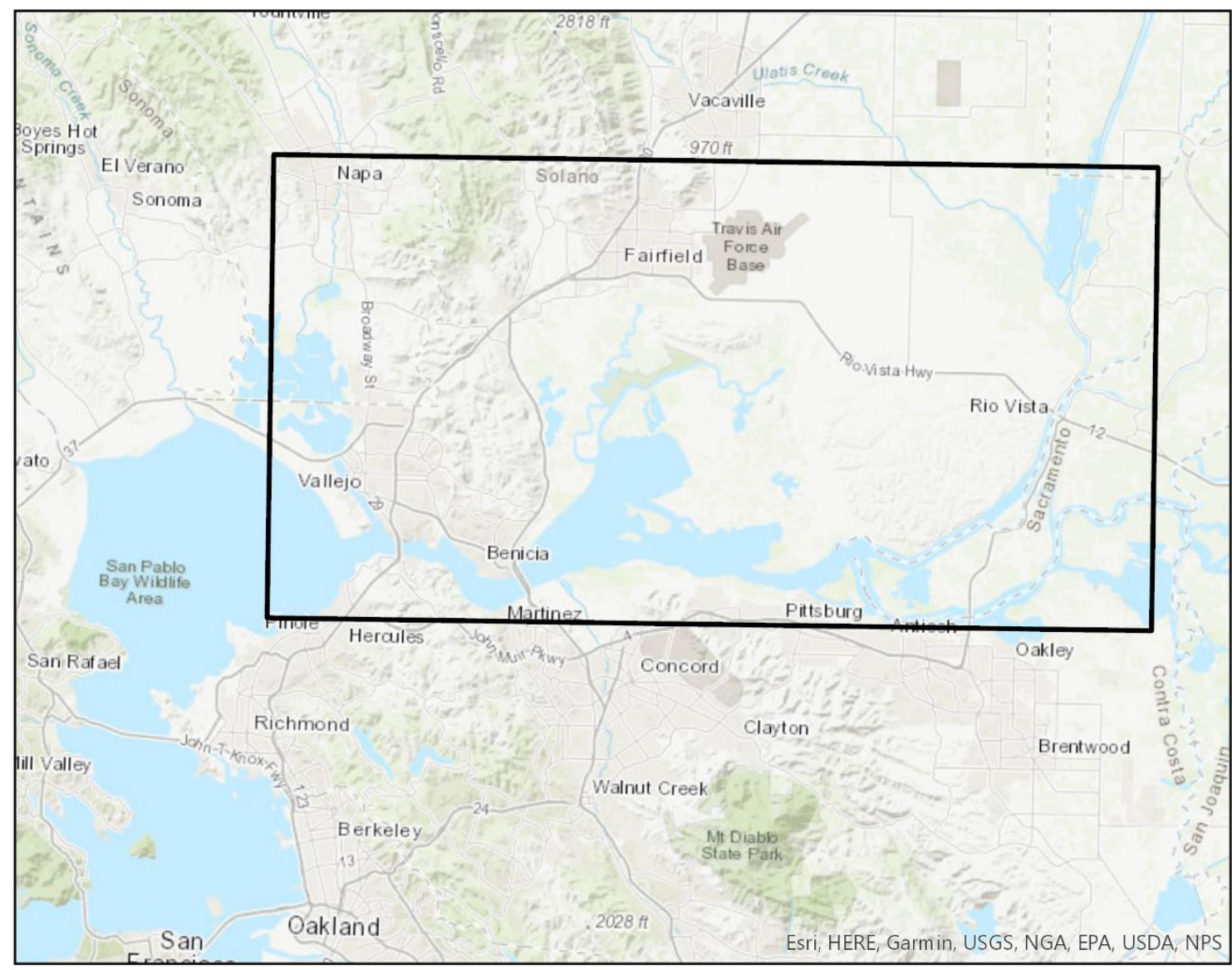

Figure 6-11: South of Fairfield Study Area

Figure 6-12 shows the original feature count (i.e. "Number of Polygons"), the total area in unit, and the total vertex count for the original wetlands layer as clipped to the "South of Fairfield" study area.

\begin{tabular}{|l|r|}
\hline \multicolumn{2}{|c|}{ Original Wetland Data in Study Area } \\
\hline Number of Polygons & 12,958 \\
\hline Total Area & $940,630,230.73$ \\
\hline Number of Vertices & $2,691,490$ \\
\hline
\end{tabular}

Figure 6-12: Statistics for Original Wetland Data in the Study Area 
Figure 6-13 shows a comparison of the number of features (i.e. polygons), the total area (units), and the total vertex count of the simplified wetland areas created with the Aggregate Polygon tool, using different aggregation distances. In addition, none of the Aggregate Polygon iterations detailed in Figure 6-13 were given parameter values for minimum area and minimum hole size, which meant that the default was zero, so that no area would be left out of the final wetland layer.

\begin{tabular}{|l|r|}
\hline \multicolumn{2}{|c|}{$\mathbf{5 0 0} \mathbf{~ m}$ Aggregation Distance } \\
\hline Number of Polygons & 16 \\
\hline Percent Change & $\mathbf{9 9 . 8 8}$ \\
\hline Total Area & $1,322,855,566.32$ \\
\hline Percent Change & $\mathbf{4 0 . 6 4}$ \\
\hline Number of Vertices & 103,046 \\
\hline Percent Change & $\mathbf{9 6 . 1 7}$ \\
\hline
\end{tabular}

\begin{tabular}{|l|r|}
\hline \multicolumn{2}{|c|}{$200 \mathbf{m}$ Aggregation Distance } \\
\hline Number of Polygons & 113 \\
\hline Percent Change & $\mathbf{- 9 9 . 1 3}$ \\
\hline Total Area & $\mathbf{1 2 . 0 4}$ \\
\hline Percent Change & 338,896 \\
\hline Number of Vertices & $\mathbf{- 8 7 . 4 1}$ \\
\hline Percent Change & \\
\hline
\end{tabular}

\begin{tabular}{|lr|}
\hline \multicolumn{2}{|c|}{$400 \mathbf{m}$ Aggregation Distance } \\
\hline Number of Polygons & 27 \\
\hline Percent Change & $\mathbf{9 9 . 7 9}$ \\
\hline Total Area & $\mathbf{3 2 . 5 3}$ \\
\hline Percent Change & 144,736 \\
\hline Number of Vertices & $\mathbf{- 9 4 . 6 2}$ \\
\hline Percent Change & \\
\hline
\end{tabular}

\begin{tabular}{|l|r|}
\hline \multicolumn{2}{|c|}{$150 \mathrm{~m}$ Aggregation Distance } \\
\hline Number of Polygons & 172 \\
\hline Percent Change & $\mathbf{- 9 8 . 6 7}$ \\
\hline Total Area & $\mathbf{7 . 4 1}$ \\
\hline Percent Change & 416,030 \\
\hline Number of Vertices & $\mathbf{8 4 . 5 4}$ \\
\hline Percent Change & \\
\hline
\end{tabular}

\begin{tabular}{|lr|}
\hline \multicolumn{2}{|c|}{350 m Aggregation Distance } \\
\hline Number of Polygons & 38 \\
\hline Percent Change & $\mathbf{9 9 . 7 1}$ \\
\hline Total Area & $\mathbf{9 7 2}, 202,387,527.82$ \\
\hline Percent Change & $\mathbf{2 7 . 8 3}$ \\
\hline Number of Vertices & 175,999 \\
\hline Percent Change & $\mathbf{9 3 . 4 6}$ \\
\hline
\end{tabular}

\begin{tabular}{|l|r|}
\hline \multicolumn{2}{|c|}{$100 \mathbf{m}$ Aggregation Distance } \\
\hline Number of Polygons & 334 \\
\hline Percent Change & $\mathbf{9 7 . 4 2}$ \\
\hline Total Area & $\mathbf{9 7 9 , 9 5 1 , 7 1 1 . 1 2}$ \\
\hline Percent Change & $\mathbf{4 . 1 8}$ \\
\hline Number of Vertices & 490,852 \\
\hline Percent Change & $\mathbf{- 8 1 . 7 6}$ \\
\hline
\end{tabular}

\begin{tabular}{|lr|}
\hline \multicolumn{2}{|c|}{$300 \mathrm{~m}$ Aggregation Distance } \\
\hline Number of Polygons & 54 \\
\hline Percent Change & $\mathbf{9 9 . 5 8}$ \\
\hline Total Area & $\mathbf{2 2 . 9 2}$ \\
\hline Percent Change & 213,860 \\
\hline Number of Vertices & $\mathbf{9 2 . 0 5}$ \\
\hline Percent Change & $1,156,256,971.20$ \\
\hline
\end{tabular}

\begin{tabular}{|lr|}
\hline \multicolumn{2}{|c|}{$\mathbf{5 0} \mathbf{~ m}$ Aggregation Distance } \\
\hline Number of Polygons & 543 \\
\hline Percent Change & $\mathbf{9 5 . 8 1}$ \\
\hline Total Area & $\mathbf{9 5 8 , 4 1 7 , 3 9 3 . 6 3}$ \\
\hline Percent Change & $\mathbf{1 . 8 9}$ \\
\hline Number of Vertices & 566,189 \\
\hline Percent Change & $\mathbf{7 8 . 9 6}$ \\
\hline
\end{tabular}

\section{Figure 6-13: Comparison of the Effects of Aggregation Distance Thresholds}

As can be seen in the tables in Figure 6-13, even a 50 meter aggregation distance results in a substantial decrease in the total number of features, which helps in data processing and in viewing the layer on the web. The number of vertices-another factor that prevented the original wetland data from being usable on the web-also decreases significantly with the aggregation tool. Higher aggregation distances however also mean a greater increase in area-or in other words a decrease in accuracy on what is technically a wetland, according to the dataset. Some increase in area is not necessarily a problem, since habitat connectivity is an important principle in wetland conservation, but it is important to choose an appropriate aggregation distance, which is why this analysis 
on the effect of wetland aggregation distances was performed. The graph in Figure 6-14 illustratess the relationship between increase in area and decrease in vertex count with changing aggregation distances. It appears that ratio between gaining simplification benefit and loosing data accuracy decreases with the aggregation distance. This suggests that a small aggregation distance is preferred when technology allows.

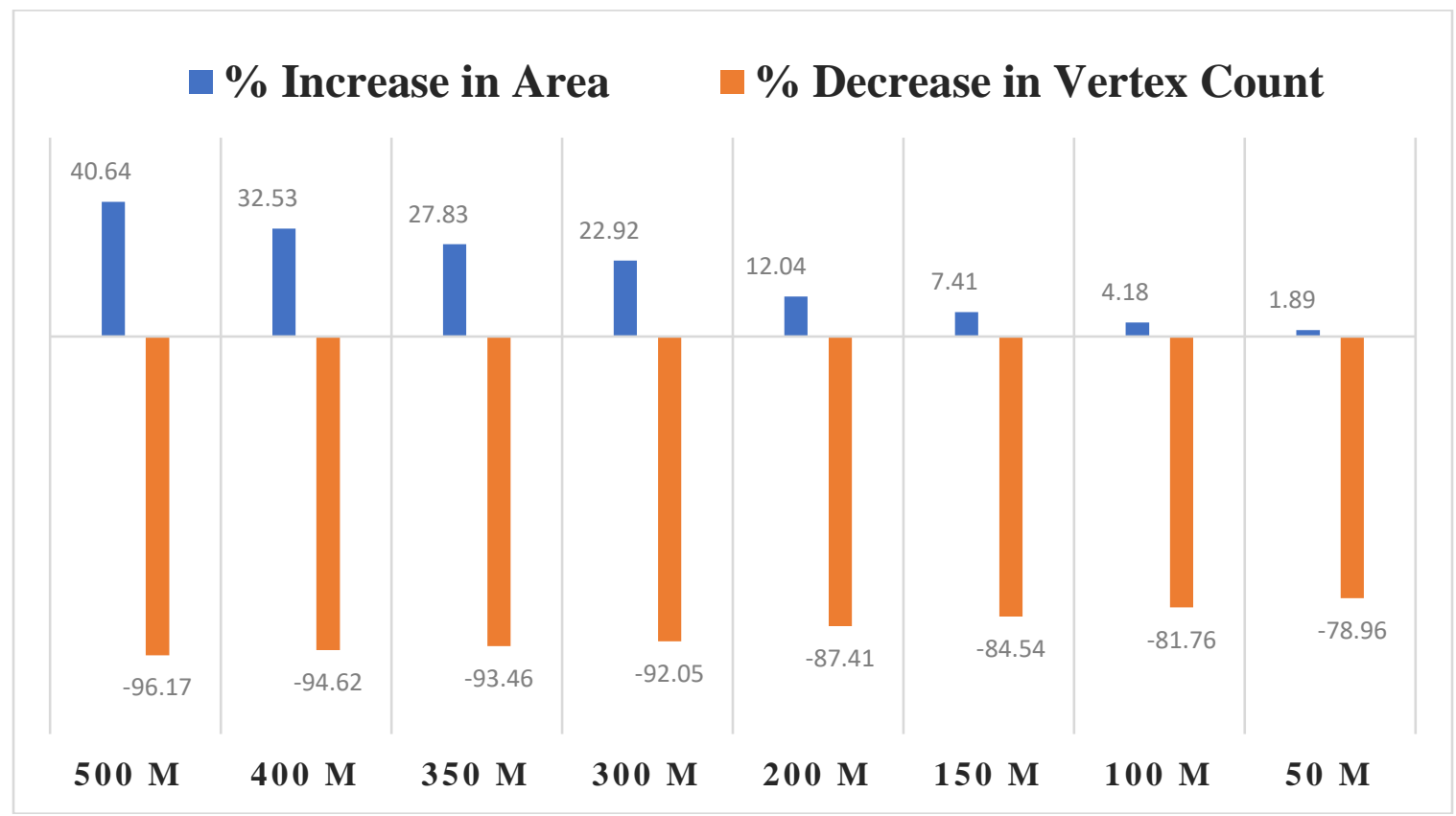

\section{Figure 6-14: Comparison of the Increase in Wetland Area and Decrease in Vertex Count with Aggregation Distance}

The maps in Figures 6-15, on the other hand, offer visual comparisons of the effects of different aggregation distances on a wetland area just south of Travis Air Force Base in the study area. It is quite obvious that a smaller distance parameter, such as 50 or 100 meters, largely honor the original wetlands delineation. 

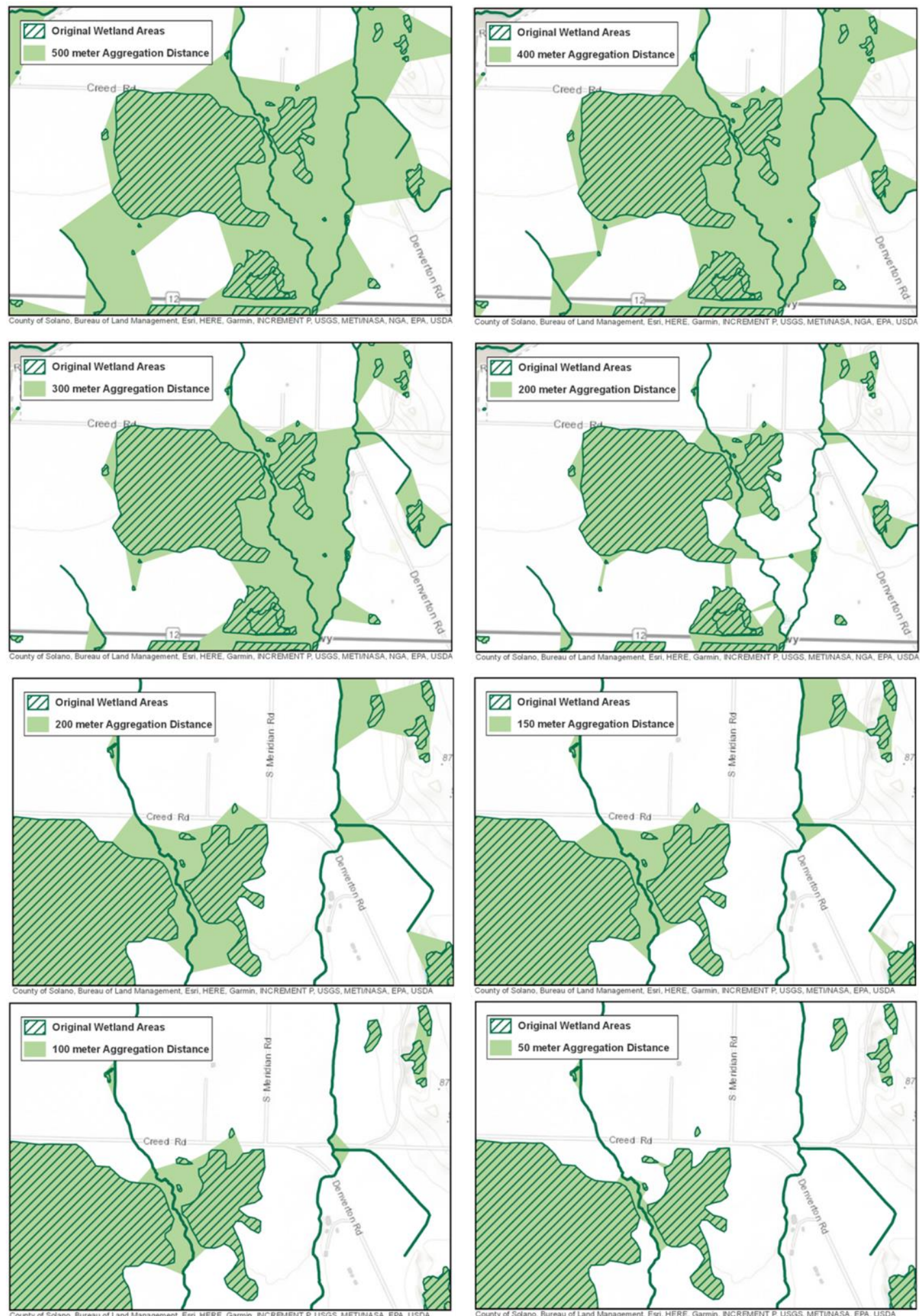

Figure 6-15: Change in Estimated Wetland Area with Aggregation Distances of 500, 400,300 and 200 meters in one area, and 200, 150, 100 and 50 meters in a smaller sub-region. 
To conclude this discussion on aggregation distances, when the wetland prioritization process is redone and published as a geoprocessing service on the USFWS server, it is suggested that the minimum area and minimum hole size be kept at the default of 0 , and that the aggregation distance be preferably less than more, depending on the capabilities of the server. When performing the prioritization analysis in ArcGIS Pro, a smaller aggregation distance is suggested (such as 150 meters), although the exact aggregation distance should be decided upon between the decision makers performing the analysis.

\subsubsection{Integration of Fragmentation and Connectivity Models}

A third suggestion to improve the scientific robustness and validity of this prioritization analysis, as noted by a collaborator of the client, Serene Ong, is to take a deeper look at how patch size, "habitat fragmentation, population fragmentation, ecosystem function and connectivity" could be integrated into the analysis. This is an important suggestion for the analysis because according to Ong there are already specific software packages out there that allow a deeper study of these factors, and integrating these software packages into the analysis would be a useful way to integrate some of the more complex (but important) scientific principles that are traditionally harder to integrate into a prioritization analysis like this.

According to Ong, there are specific software packages out there that allow a deeper study of these factors (Serene Ong, personal communication, 2018). It would be useful to look into the possibility of integrating some of these software packages into future prioritization analyses.

\subsection{Summary}

In conclusion, this project created a Wetland Prioritization Toolbox for the USFWS to enable their GIS employees to quickly perform and re-do wetland prioritization analyses for Cade London, who is in charge of designating wetlands in the United States that are of international importance. This project then created a web application to be used by Cade and other decision makers whom he collaborates with, to gather different perspectives on wetland priorities. The products created by the project will enable Cade London and his associate employees in the USFWS to quickly repeat wetland prioritization analyses with different criteria and compare the results. In automating the analysis process into one easy workflow, this project has immense amounts of potential value not only to employees in the USFWS, but for environmental and conservation managers and decision makers in general, as it enables scientists and other decision makers to approach prioritization analyses in a multiple-criteria decision analysis framework, without needing to deal with the background GIS work. With that said, there are a few recommended actions that should be taken before large scale application of the deliverables produced by this project, including integrating additional datasets into the analysis, conducting further research on distance and area thresholds to use in the analysis and changing the parameter values for the aggregate polygons tool in particular, and performing further research into how to integrate habitat ecosystem connectivity models into this analysis. 


\section{Chapter 7 - Conclusions and Future Work}

This chapter gives a brief overview of what this project accomplished and ends with suggestions for future projects.

\subsection{Project Summary}

This project addressed a need to automate wetland prioritization analyses into a condensed GIS workflow, as well as to condense it into an interface where decision makers and stakeholders could re-do wetland prioritization analyses with their own weights of importance without needing to understand GIS, and to share the results.

To address these needs, an ArcGIS Pro Toolbox automating a wetland prioritization analysis (and automating the accompanying data preparation steps) was created. In addition, a custom geoprocessing service was created and published, and integrated into a web map, where users who were unfamiliar with or did not have access to GIS could redo the wetland prioritization analysis with their own weights of importance inside of a web browser. All the client's functional and non-functional requirements, as listed in the beginning of this document, were satisfied by this project.

In simplifying the wetland prioritization analysis process into a custom ArcGIS Pro Toolbox and web application, this project has immense value not only to employees in the USFWS, but also for environmental and conservation managers and decision makers in general. With the prioritization analysis process simplified into one easy workflow, scientists and decision makers in the USFWS can now repeat prioritization analyses with minimal cost and effort, enabling them to quickly gather information on the wetland priorities of other stakeholders, without those stakeholders needing to understand anything about GIS.

\subsection{Future Work}

There is ample room for future projects to expand on this project. One such future project could be to research how to best integrate the concepts of habitat and ecosystem connectivity into the analysis, and in particular, how to translate over scientific models on connectivity that have already been created, into this analysis. To do so, a person would need to study and become familiar with connectivity models being used in current scientific literature, and then figure out how to integrate those models into the geoprocessing tool. It is possible that some of the models are written in R, in which case the student would also have to learn how to integrate those models into a python framework, which is the language used by geoprocessing services (ArcMap) and web tools (ArcGIS Pro) in Esri software (which is what was used for this project). Another suggestion for future work would be to complete a more extensive literature review on how scientists (not just managers and decision makers) currently prioritize wetlands, by completing a review as might be published in a scientific journal. The next step would be to then re-do the project with a focus on tweaking the analysis to integrate the common themes found in that literature review. Such a project as this could then be published in a journal and would be a great way to help this project and its ideas be integrated into, and accepted by, the scientific community. Finally, additional work that could be done on this 
project would be to integrate more factors into the analysis (such as climate change projections, presence of protected lands, water quality data, etc.) and to re-do the analysis for different states. 


\section{Works Cited}

Andersen, A. N., Hoffmann, B. D., Muller, W. J., \& Griffiths, A. D. (2002). Using ants as bioindicators in land management: simplifying assessment of ant community responses. Journal of Applied Ecology, 39(1), 8-17. https://doi.org/10.1046/j.13652664.2002.00704.x

Arlettaz, R., Schaub, M., Fournier, J., Reichlin, T. S., Sierro, A., Watson, J. E. M., \& Braunisch, V. (2010). From publications to public actions: When conservation biologists bridge the gap between research and implementation. BioScience, 60(10), 835-842. https://doi.org/10.1525/bio.2010.60.10.10

Bried, J. T., Herman, B. D., \& Ervin, G. N. (2007). Umbrella potential of plants and dragonflies for wetland conservation: a quantitative case study using the umbrella index. Journal of Applied Ecology, 44(4), 833-842. https://doi.org/10.1111/j.13652664.2007.01299.x

Copeland, H. E., Tessman, S. A., Girvetz, E. H., Roberts, L., Enquist, C., Orabona, A., ... Kiesecker, J. (2010). A geospatial assessment on the distribution, condition, and vulnerability of Wyoming's wetlands. Ecological Indicators, 10(4), 869-879. https://doi.org/10.1016/j.ecolind.2010.01.011

Feick, R. D., \& Hall, G. B. (2001). Balancing consensus and conflict with a GIS-based multi-participant, multi-criteria decision support tool. GeoJournal, 53(4), 391-406. Retrieved from https://search-proquestcom.ezproxy.redlands.edu/pqrl/docview/223678012/fulltextPDF/1DD84690591149 35PQ/1? accountid=14729

Jankowski, P. (1995). Integrating geographical information systems and multiple criteria decision-making methods. International Journal of Geographical Information Systems, 9(3), 251-271. https://doi.org/10.1080/02693799508902036

Kiester, A. R., Scott, J. M., Csuti, B., Noss, R. F., Butterfield, B., Sahr, K., \& White, D. (1996). Conservation prioritization using GAP data. Conservation Biology, 10(5), 1332-1342. https://doi.org/10.1046/j.1523-1739.1996.10051332.x

McInnes, R. J. (2016). Wetlands and flood management: natural hazard regulation. In The Wetland Book (pp. 1-7). Dordrecht: Springer Netherlands. https://doi.org/10.1007/978-94-007-6172-8_216-1

Ming, J., Xian-guo, L., Lin-shu, X., Li-juan, C., \& Shouzhenga, T. (2007). Flood mitigation benefit of wetland soil - A case study in Momoge National Nature Reserve in China. Ecological Economics, 61(2-3), 217-223. https://doi.org/10.1016/J.ECOLECON.2006.10.019

Prendergast, J. R., Quinn, R. M., \& Lawton, J. H. (1999). The gaps between theory and practice in selecting nature reserves. Conservation Biology, 13(3), 484-492. https://doi.org/10.1046/j.1523-1739.1999.97428.x

Roberge, J.-M., \& Angelstam, P. (2004). Usefulness of the umbrella species concept as a conservation tool. Conservation Biology, 18(1), 76-85. https://doi.org/10.1111/j.1523-1739.2004.00450.x

Rodgers, J. A. J., \& Schwikert, S. T. (2003). Buffer zone distances to protect foraging and loafing waterbirds from disturbance by airboats in Florida. Waterbirds: The International Journal of Waterbird Biology, 26(4), 437-443. 
https://doi.org/10.1675/1524-4695(2003)026[0437:BZDTPF]2.0.CO;2

Roe, J. H., \& Georges, A. (2006). Heterogeneous wetland complexes, buffer zones, and travel corridors: Landscape management for freshwater reptiles. Biological Conservation, 135(2007), 67-76. https://doi.org/10.1016/j.biocon.2006.09.019

Semlitsch, R. D., \& Bodie, J. R. (2003). Biological criteria for buffer zones around wetlands and riparian habitats for amphibians and reptiles. Conservation Biology, 17(5), 1219-1228. https://doi.org/10.1046/j.1523-1739.2003.02177.x

Thorhill, J. L. (2014). Bridging the gap between research and decision-making: empirical evidence from a case study of gray wolf (Canis lupus) management in the U.S. (Doctoral dissertation) George Mason University. https://doi.org/UMI Number 3625116

United Nations Educational Scientific and Cultural Organization. (1994). Convention on Wetlands of International Importance especially as Waterfowl Habitat. Ramsar, Iran: Office of International Standards and Legal Affairs, United Nations Educational, UNESCO. Retrieved from http://www.ramsar.org/sites/default/files/documents/library/current_convention_text e.pdf

US Fish and Wildlife Service. (2013). US Fish and Wildlife Service employee pocket guide: FWS fundamentals. Retrieved November 4, 2017, from https://www.fws.gov/info/pocketguide/fundamentals.html 


\section{Appendix A. Models, Scripts, and Script Tools Created in the Project}

Appendix A provides diagrams of the model and script tools used in the Wetland Prioritization Toolbox, as well as a brief overview of a few of the parameter settings that were used to publish the geoprocessing service. The first four sections in the Appendix are titled according to the name of the toolset within the toolbox (Figure A-1).

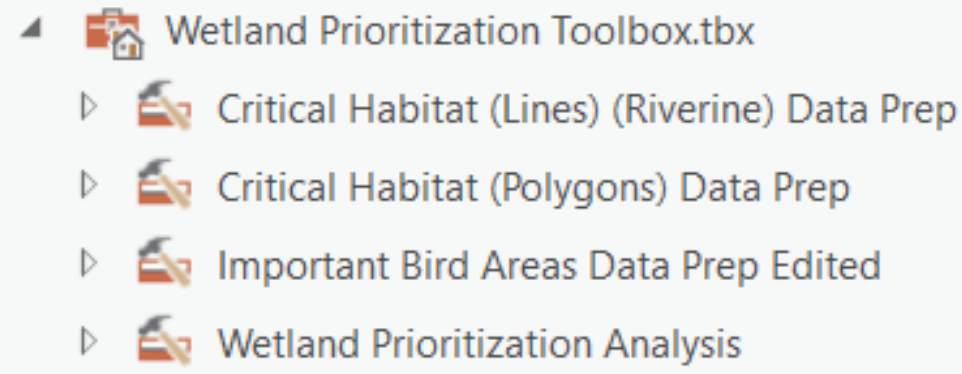

Figure A-1: Toolsets in Wetland Prioritization Toolbox

The fifth section of the Appendix then gives an overview of the script tool and model that was created in 10.5 for the geoprocessing service, and the last section, "Publishing the Geoprocessing Service," discusses a few of the parameter settings used to publish the geoprocessing service.

\section{Critical Habitat (Lines) (Riverine) Data Prep}

The "Critical Habitat (Lines) (Riverine) Data Prep" toolset consisted of four steps and a background script tool that was integrated into Step 2. Figure A-2 shows an overview of the toolset.

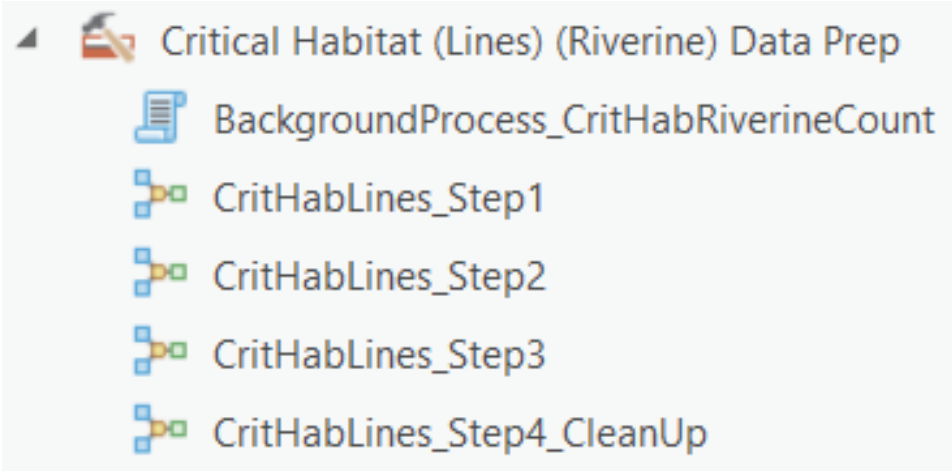

FigureA-2: Critical Habitat (Lines) (Riverine) Data Prep toolbox

Figures A-3 to A-7 show diagrams of the data preparation steps for the critical habitat lines data preparation. 


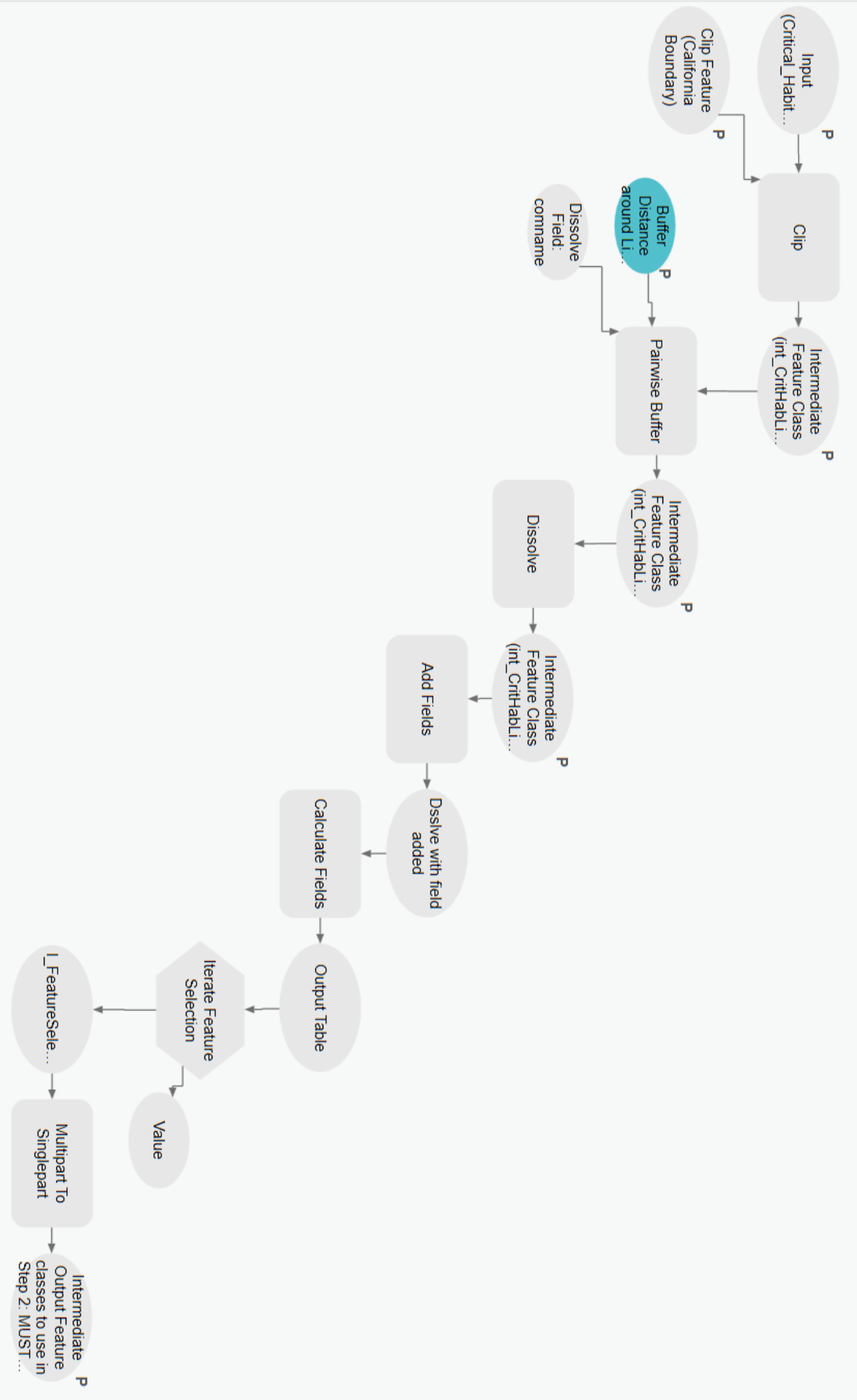

Figure A-3: CritHabLines_Step1 


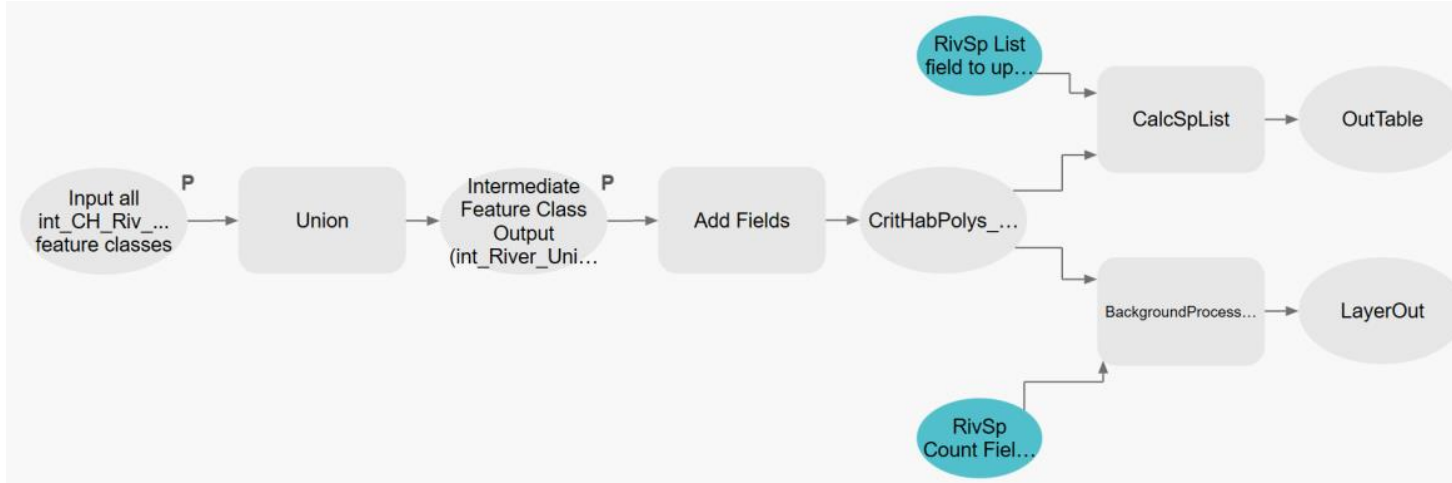

Figure A-4: CritHabLines_Step 2

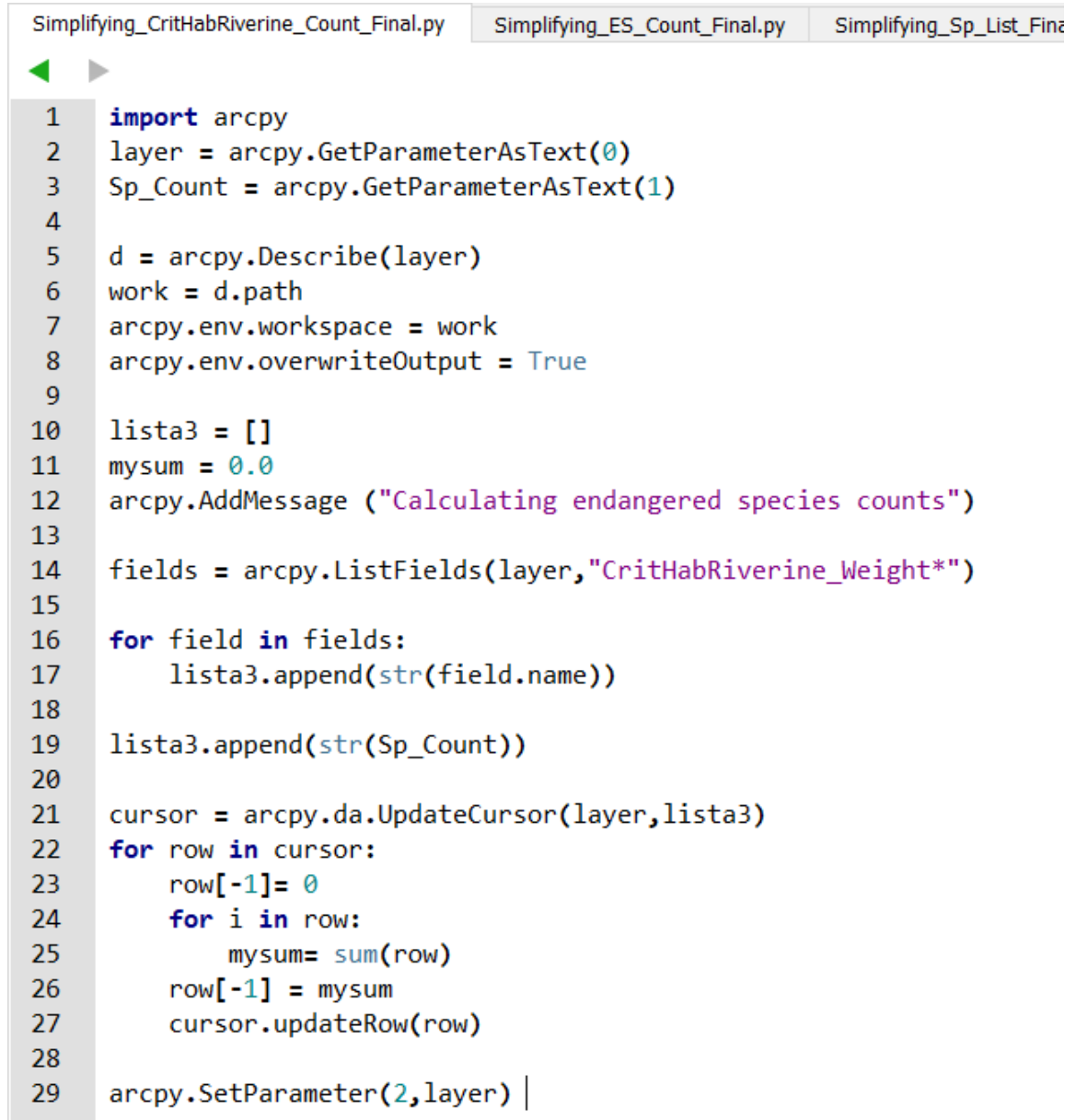

\section{Figure A-5: Background Process in Step 2}

(Simplifying_CritHabRiverine_Count_Final.py)

The Simplifying_CritHabRiverine_Count_Final.py script was integrated into a script tool such that parameter 0 and parameter 2 had data types of "Feature Class." Parameter 1 had a "Field" data type. Parameters 0 and 1 were required input parameters with Parameter 1 having dependency on parameter 0. 


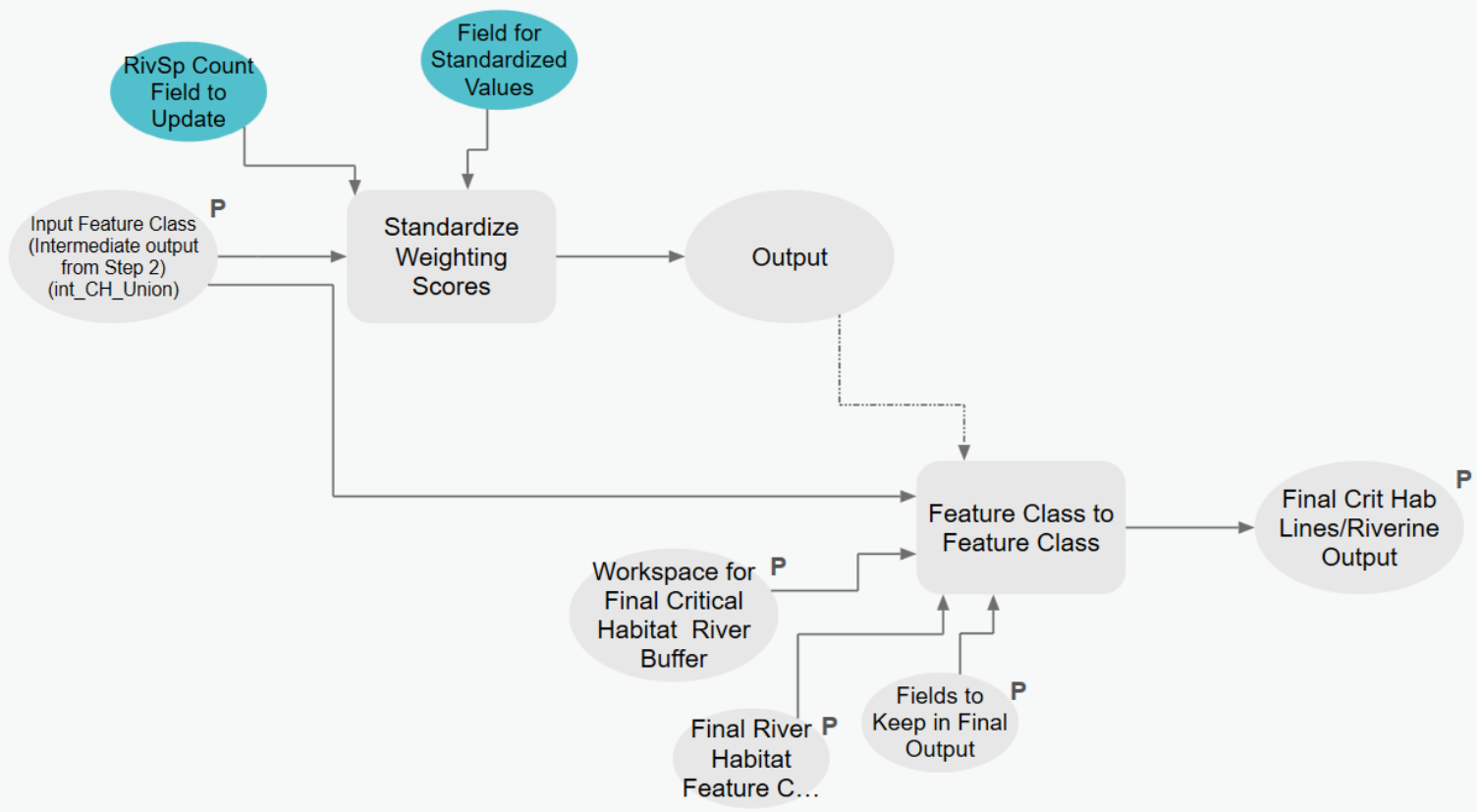

Figure A-6: CritHabLines_Step3

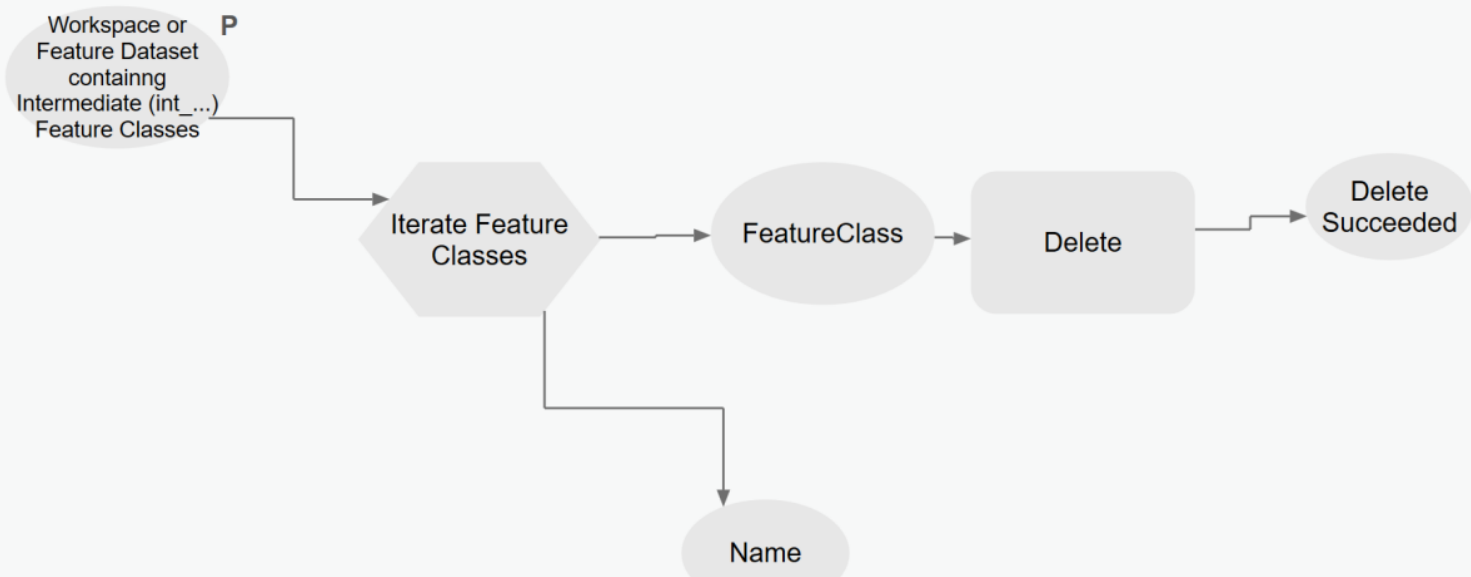

Figure A-7: CritHabLines_Step4_CleanUp

Figure A-8 and A-9 show the user interfaces for the critical habitat lines data preparation steps. 


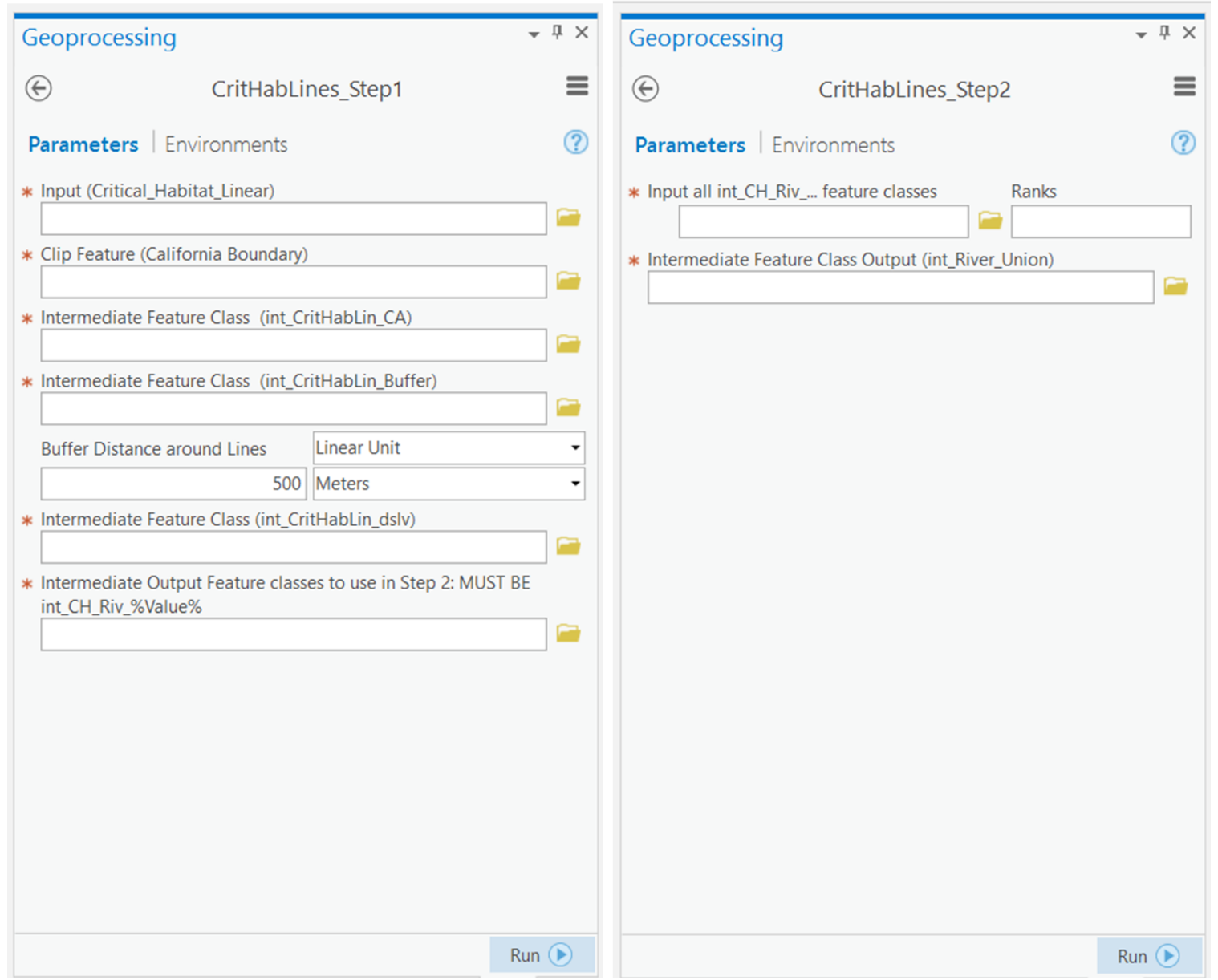

Figure A-8 Geoprocessing Pane Interface for CritHabLines_Step1 and CritHabLines_Step2 


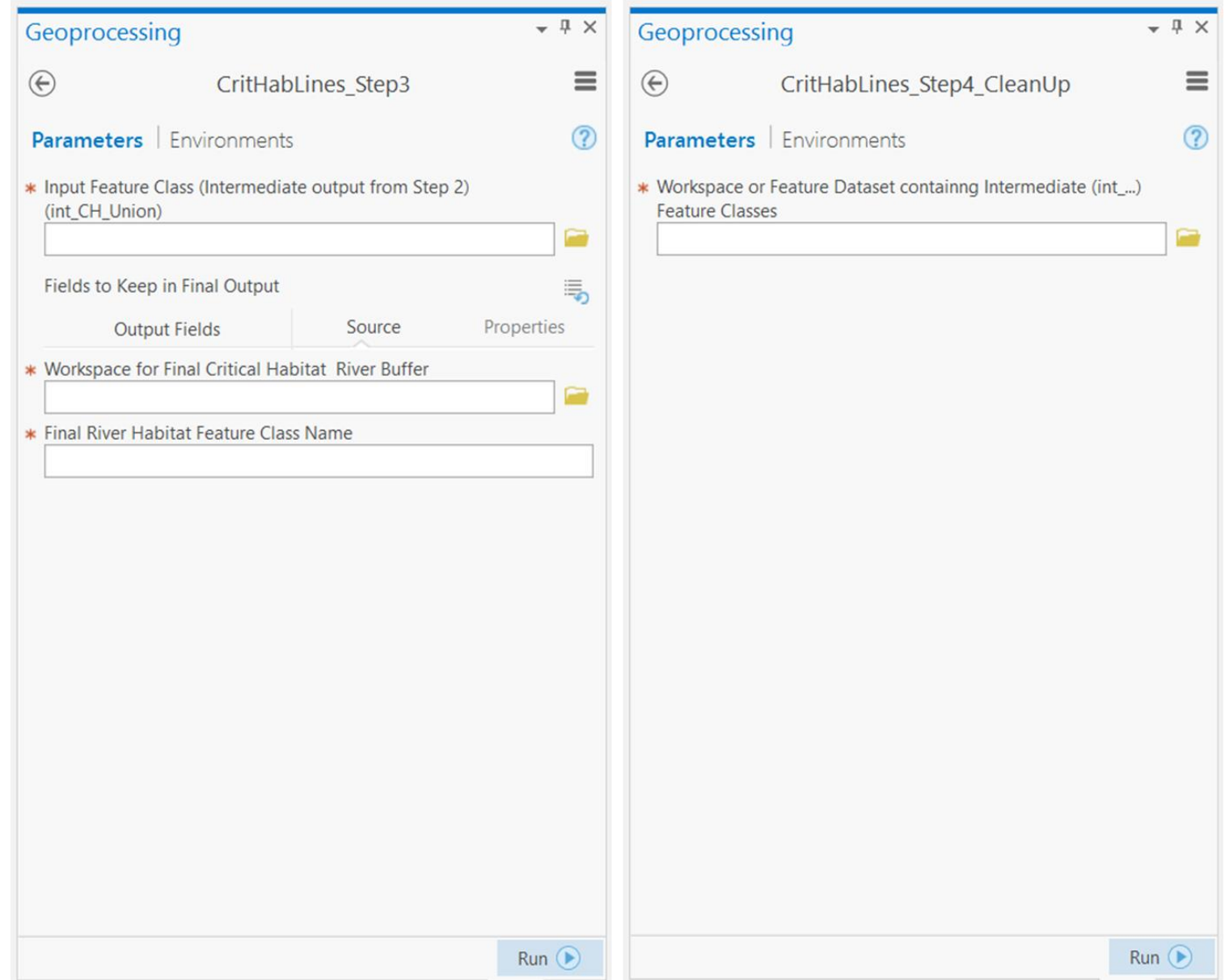

Figure A-9: Geoprocessing Pane Interface for CritHabLines_Step3 and CritHabLines_Step4_CleanUp

\section{Critical Habitat (Polygons) Data Prep}

"Critical Habitat (Polygons) Data Prep" was the second toolset in the toolbox and consisted of 5 steps and 5 background models and scripts (Figure A-10). 


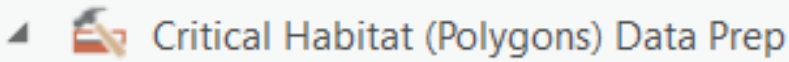

4 Eritical Habitat Prep Background Processes

言 CalcEndgSpCount

卧 CalcSpList

嘅 Calculate Threatened Species Count

Sora CritHab_Step2_background_ES

Sor CritHab_Step2_background_TS

Standardize Weighting Scores

Por CritHabPolys_Step1

Pa CritHabPolys_Step2

So CritHabPolys_Step3

SoritHabPolys_Step4

So CritHabPolys_Step5_CleanUp

\section{Figure A-10: Critical Habitat Prep Background Process}

As can be seen, the Critical Habitat (Polygons) Data Prep consisted of many more steps, as well as quite a few background scripts and models. Figures A-11 to A-21 show the models and script tools for the Critical Habitat (Polygons) Data Prep toolset.

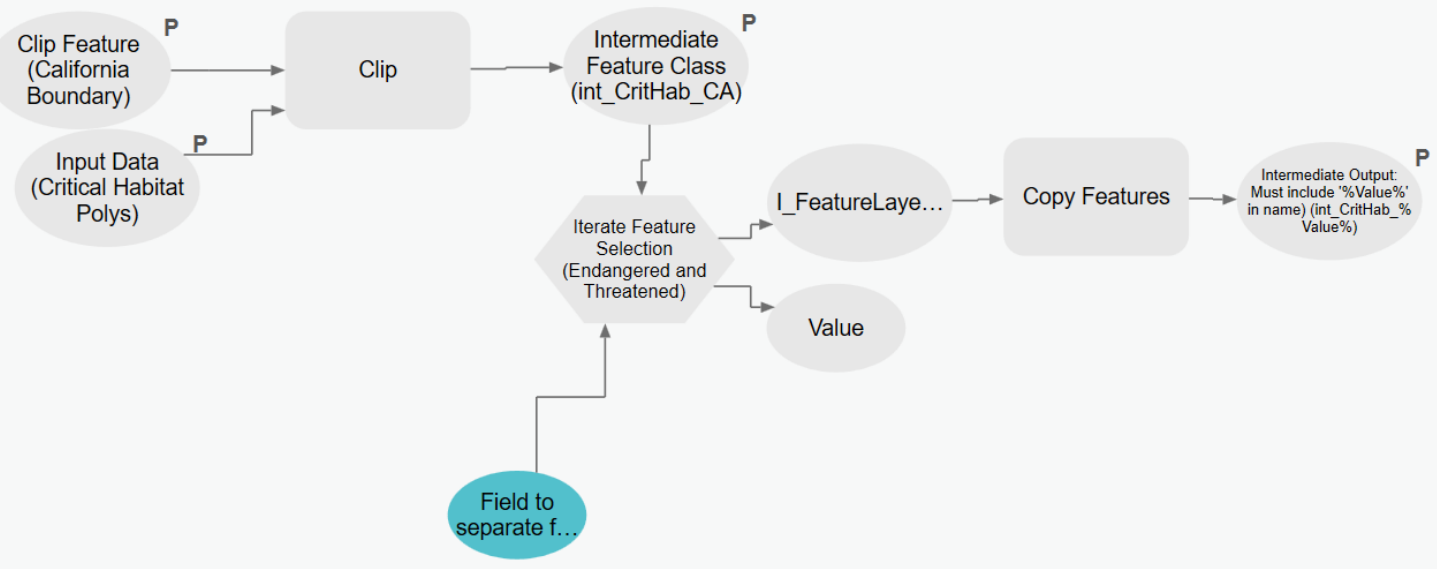

Figure A-11: CritHabPolys_Step1 

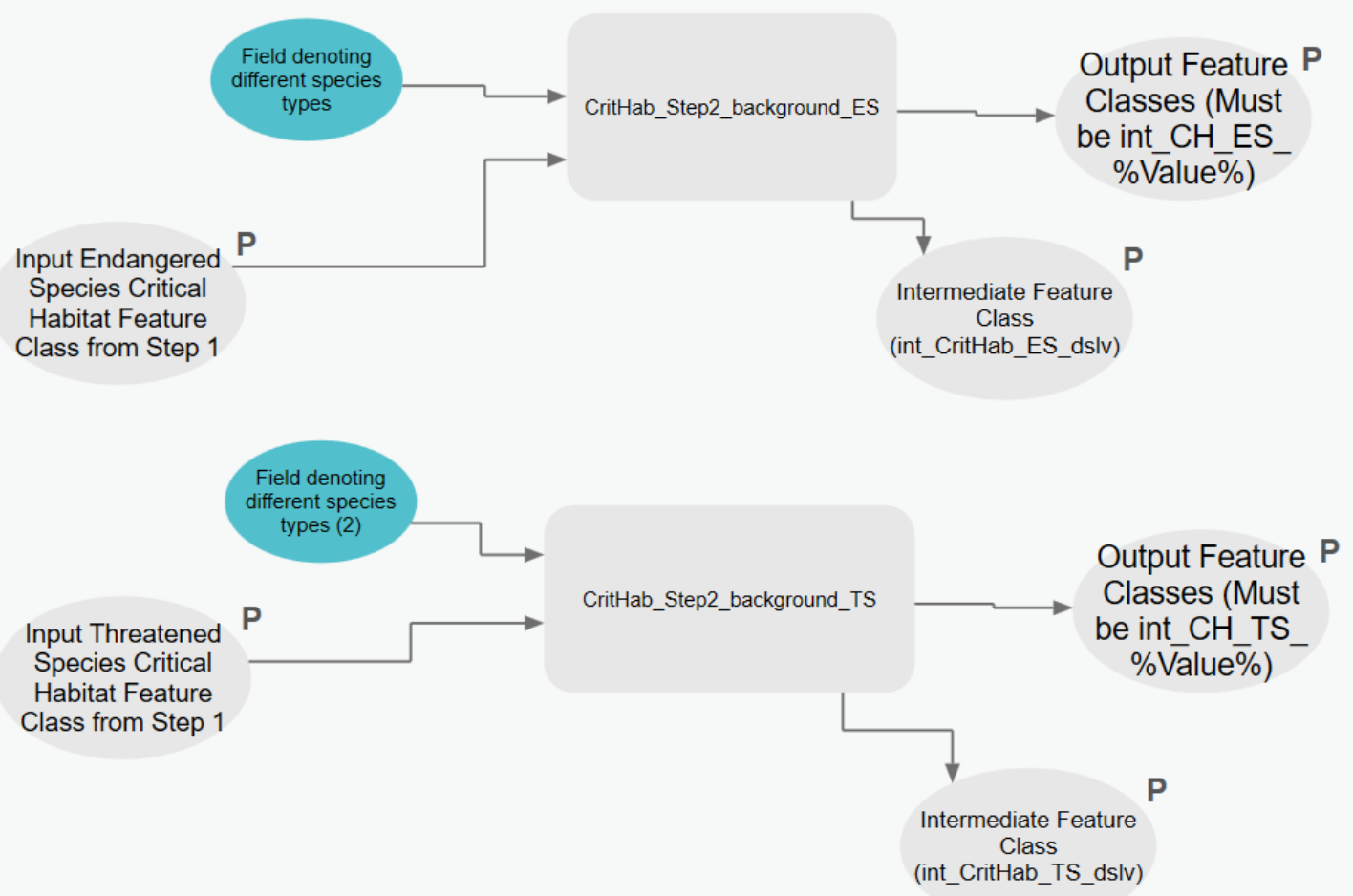

\section{Figure A-12: CritHabPolys_Step2}

CritHabPolys_Step2 was basically a model that was created to produce an easy user interface for two models that were exactly the same except for one being specific towards endangered species and adding the appropriate fields for that, and the other for threatened species. 


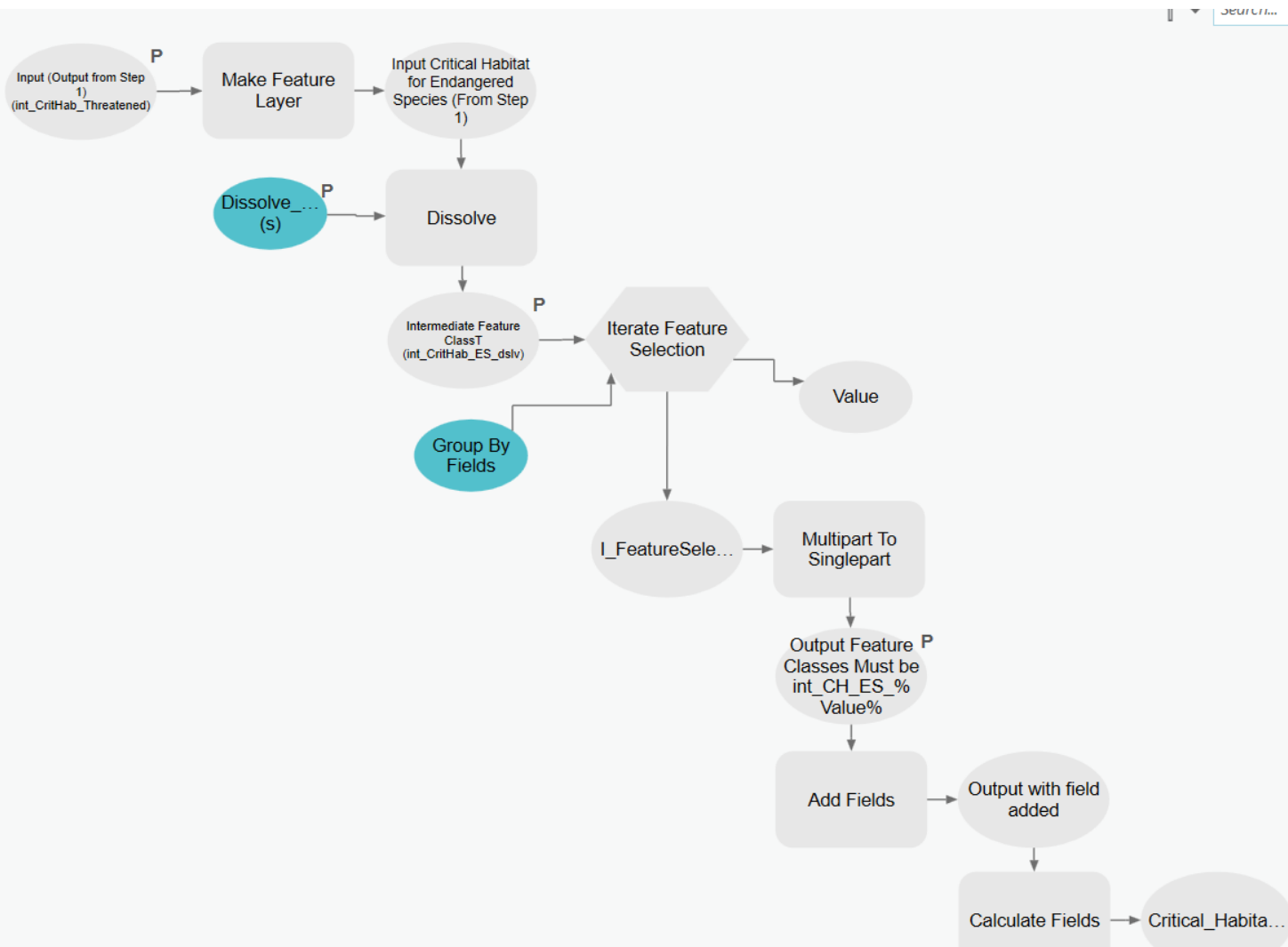

Figure A-13: CritHab_Step2_background_ES (background process)

In CritHab_Step2_background_ES, the endangered species feature class was dissolved by comname with "create multipart features" checked. The model then iterated through the features in the feature class as grouped by OBJECTID, to create separate feature classes for each endangered species range. The feature were then converted to singlepart features again. This somewhat long approach was used instead of merely applying Iterate Feature Selection on the original input endangered species feature class, because special characters in the comname field for endangered species made the comname field not usable in "Iterate Feature Selection." 


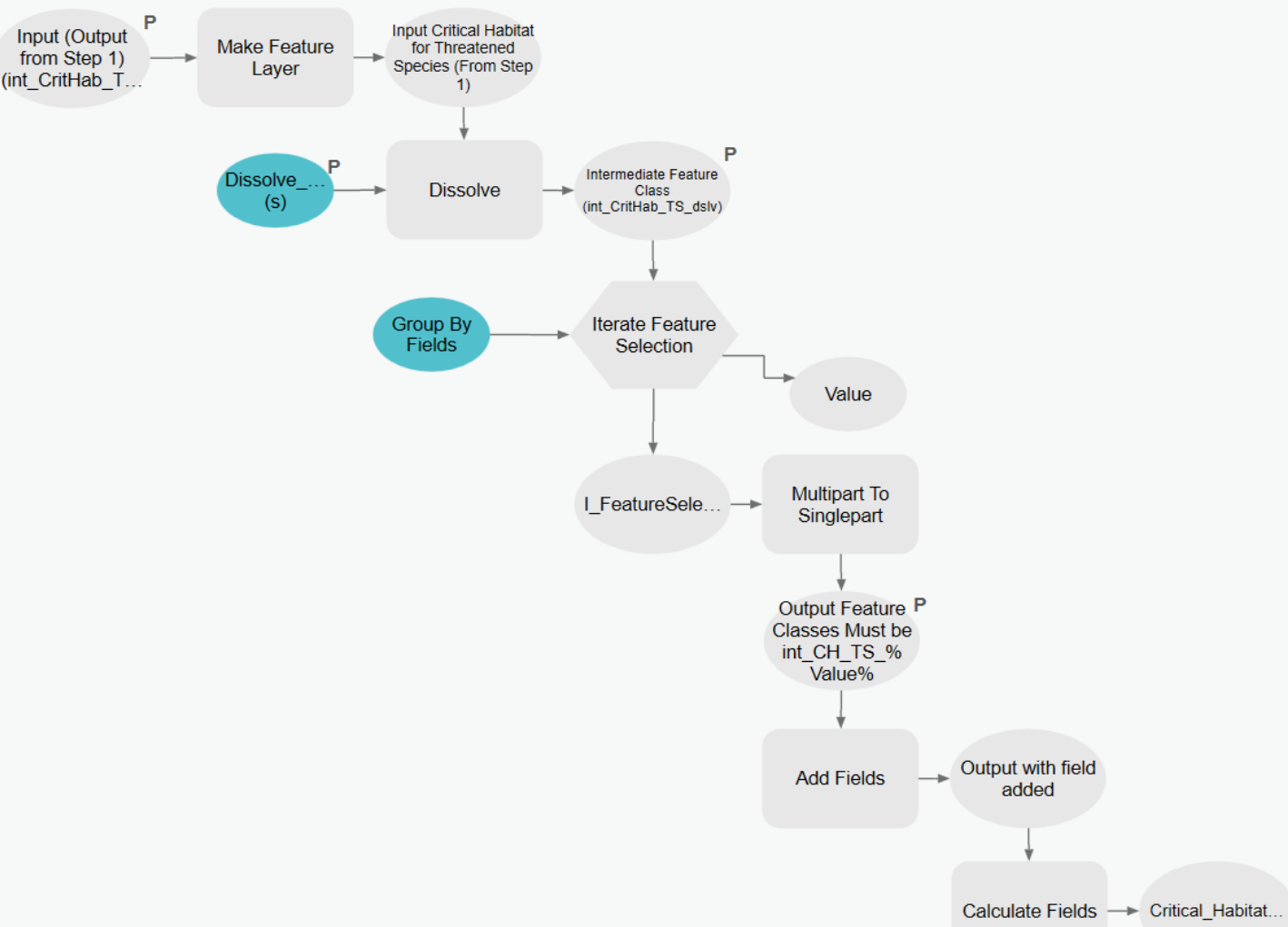

Figure A-14: CritHab_Step2_background_TS (background process)

The CritHab_Step2_background_TS model used the same approach as the model created for endangered species except that the field added was i_Threatened_Weight instead of i_Endangered_Weight. 


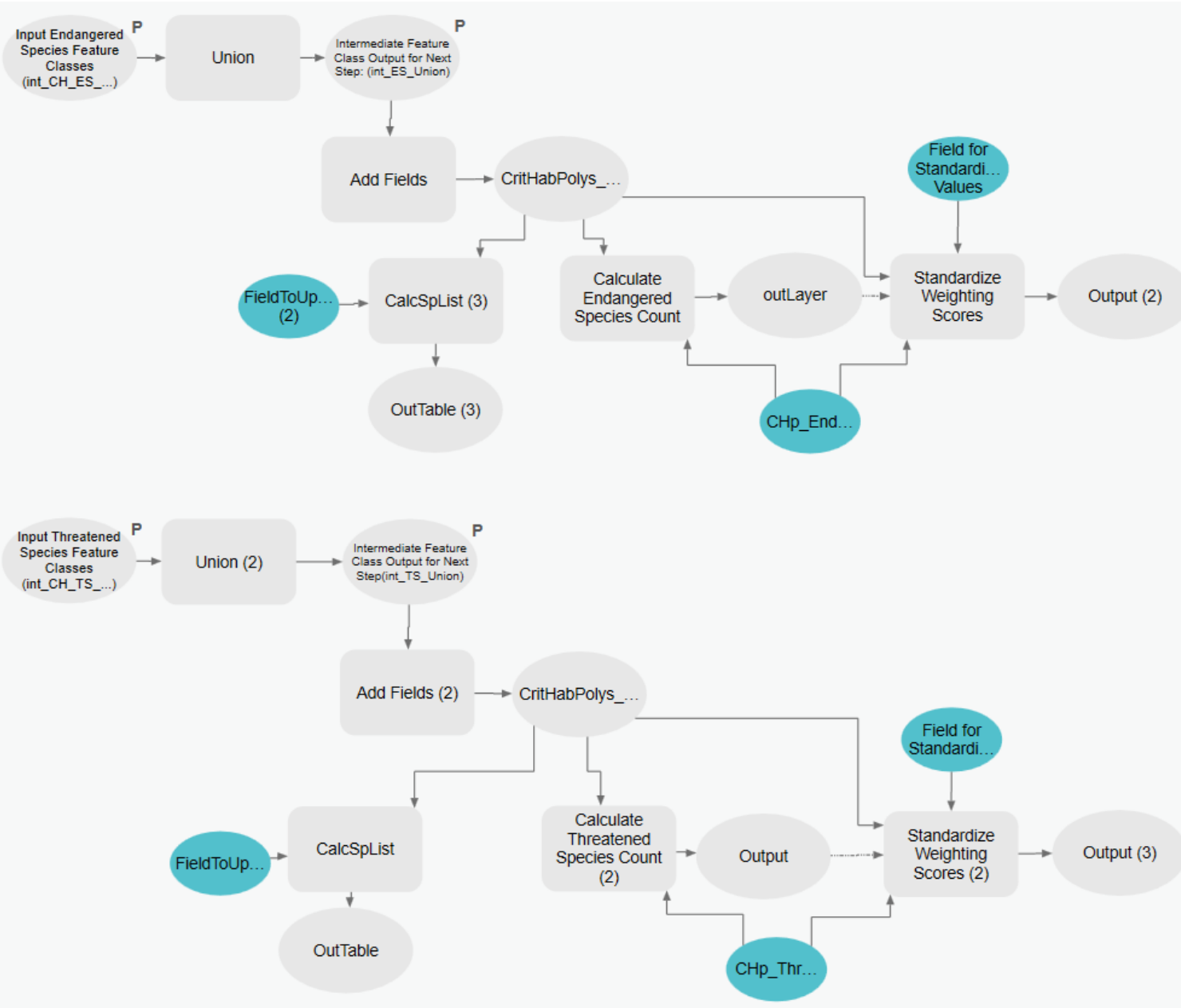

Figure A-15: CritHabPolys_Step 3

CritHabPolys_Step3 used 3 different script tools (CalSpList, Calculate Endangered Species Count, Calculate Threatened Species Count, and Standardize Weighting Scores) corresponding to the files (Simplifying_Sp_List_Final.py, Simplifying_ES_Count_Final.py, Simplifying_TS_Count_Final.py, and StandardizingWeightScores.py). 


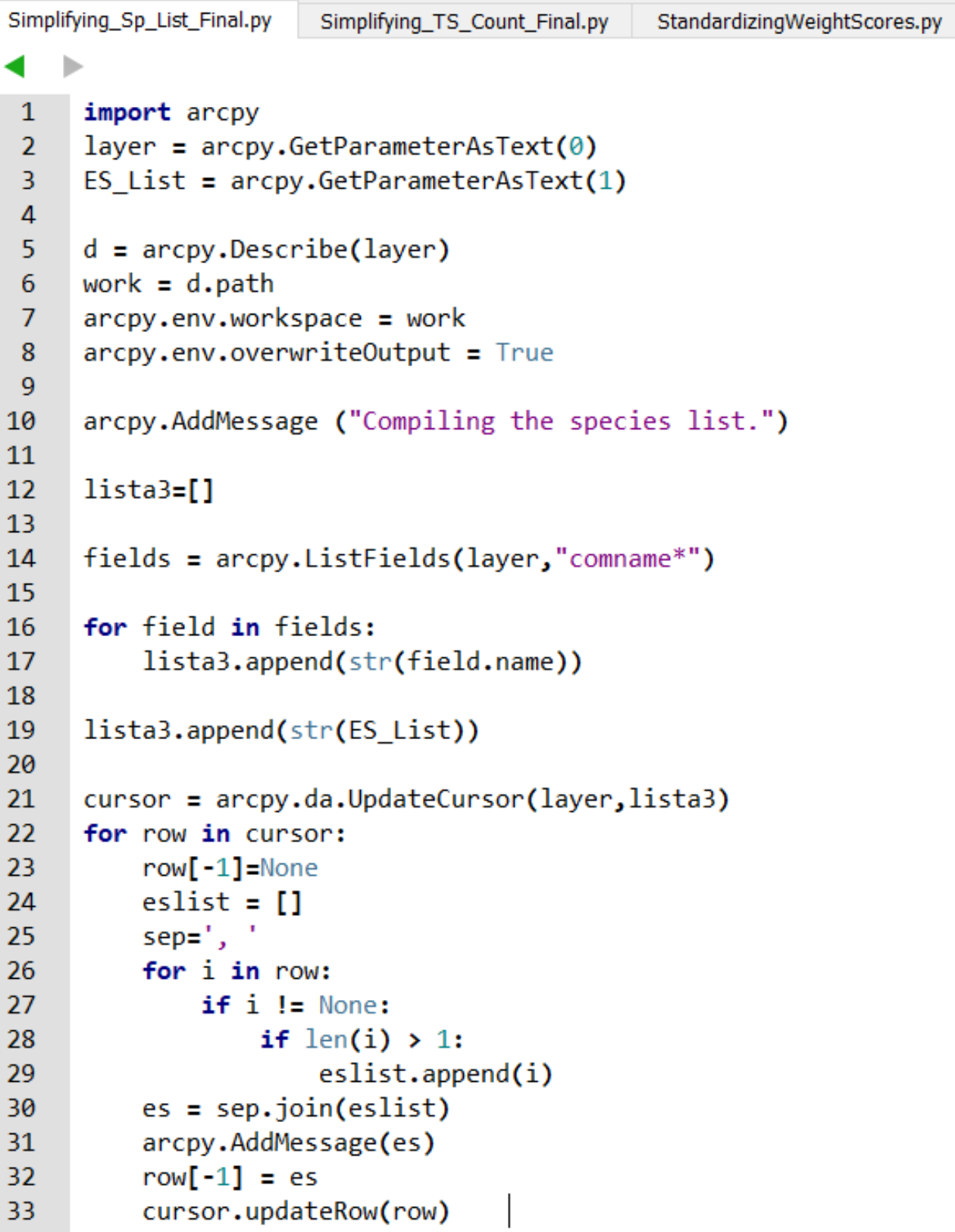

Figure A-16: CalcSpList Script Tool (Simplifying_Sp_List_Final.py) used in Step_3

The Simplifying_Sp_List_Final.py script was integrated into a script tool such that parameter 0 and parameter 2 had the data type of "Feature Layer" and parameter 1 had the data type of "Field." Parameter 0 and 1 were required inputs, with parameter 1 being dependent on parameter 0 . Parameter 2 was a derived output. 


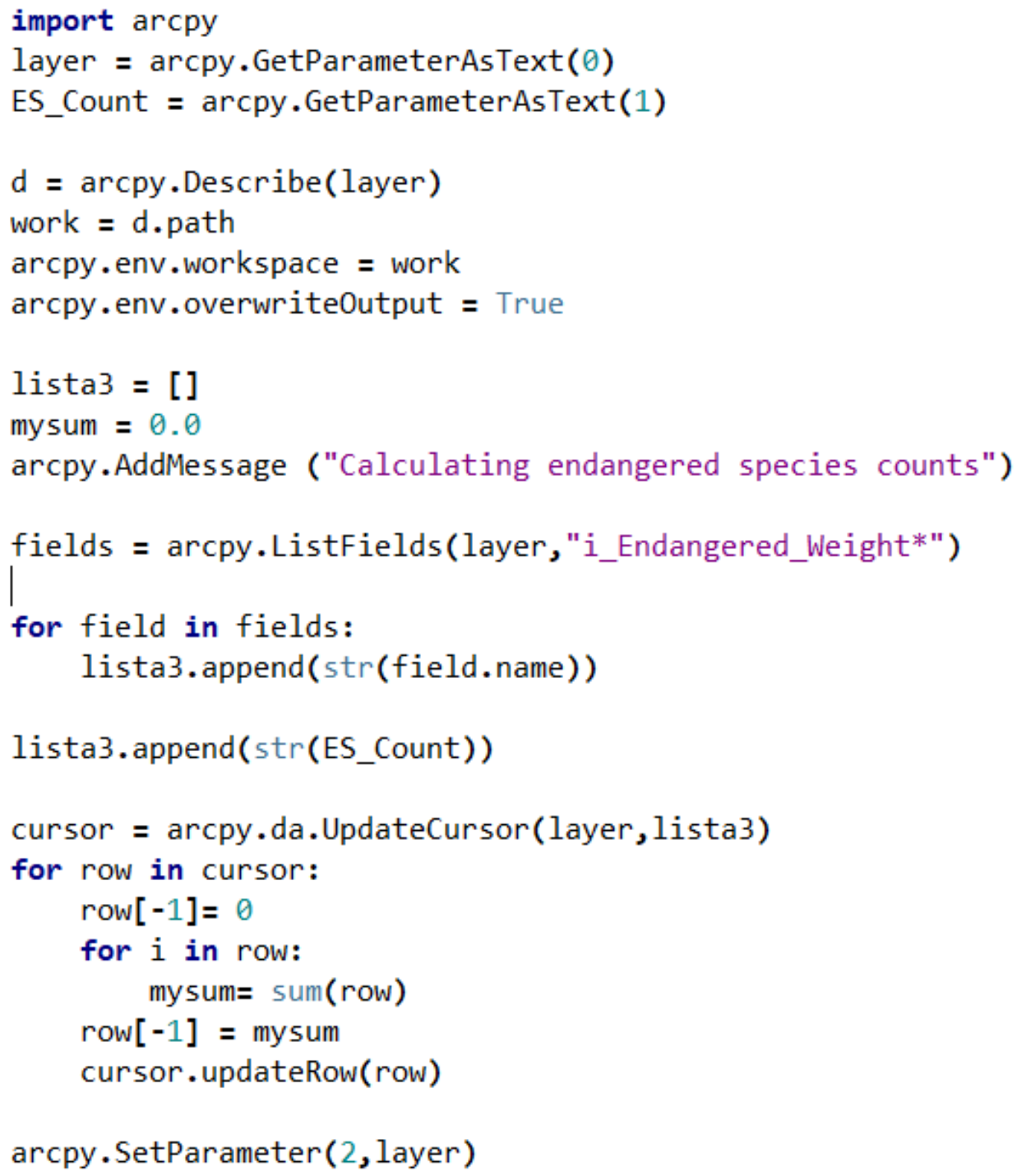

Figure A-17: Calculate Endangered Species Count Script Tool used in Step_3 (Simplifying_ES_Count_Final.py)

The Simplifying_ES_Count_Final.py script was integrated into a script tool such that parameter 0 and parameter 2 had the data type of "Feature Layer" and parameter 1 had the data type of "Field." Parameter 0 and 1 were required inputs, with parameter 1 being dependent on parameter 0 . Parameter 2 was a derived output. 


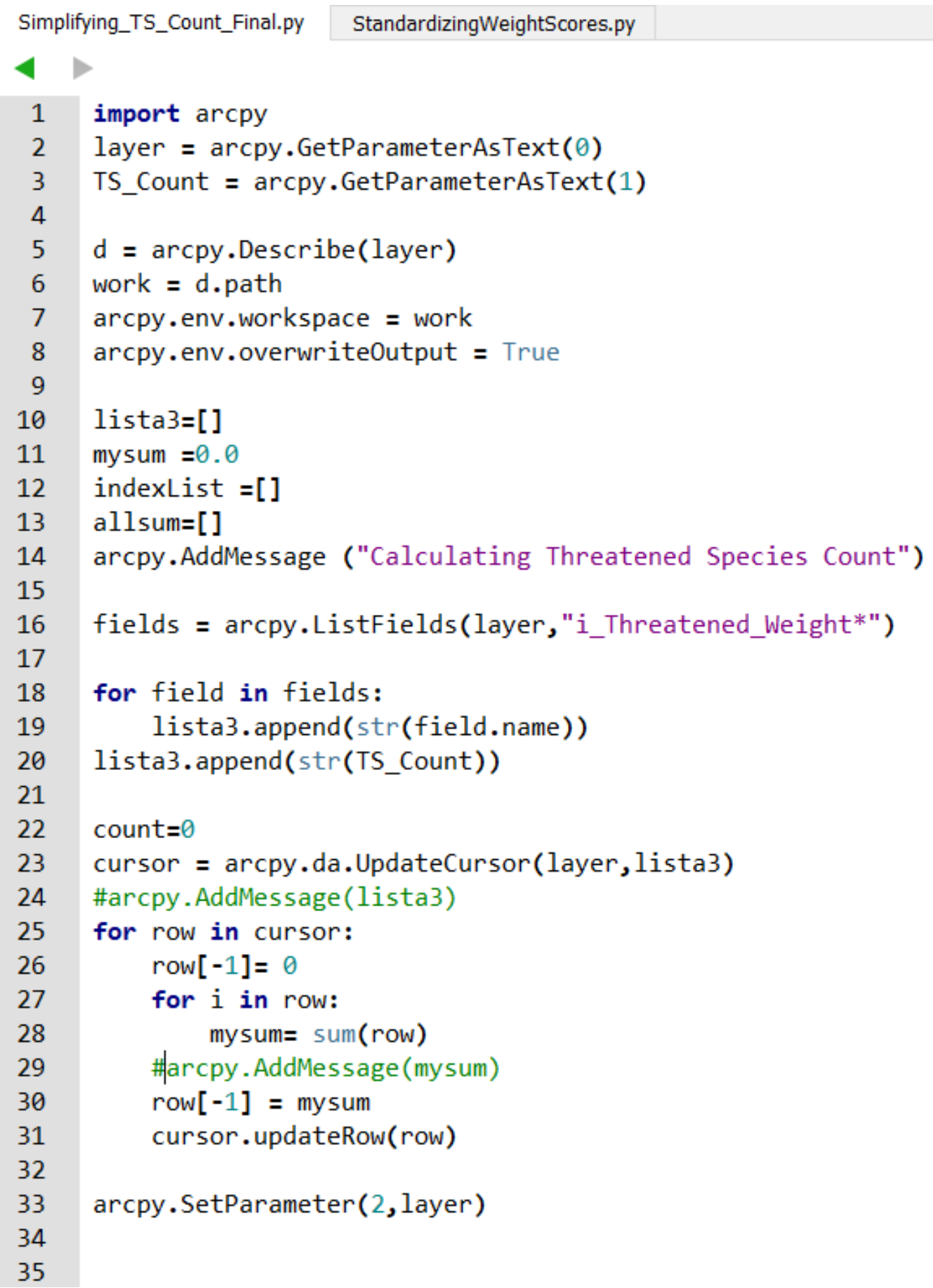

Figure A-18: Calculate Threatened Species Count Script Tool Used in Step_3 (Simplfying_TS_Count_Final.py)

The Simplifying_TS_Count_Final.py script was integrated into a script tool such that parameter 0 and parameter 2 had the data type of "Feature Layer" and parameter 1 had the data type of "Field." Parameter 0 and 1 were required inputs, with parameter 1 being dependent on parameter 0 . Parameter 2 was a derived output. 


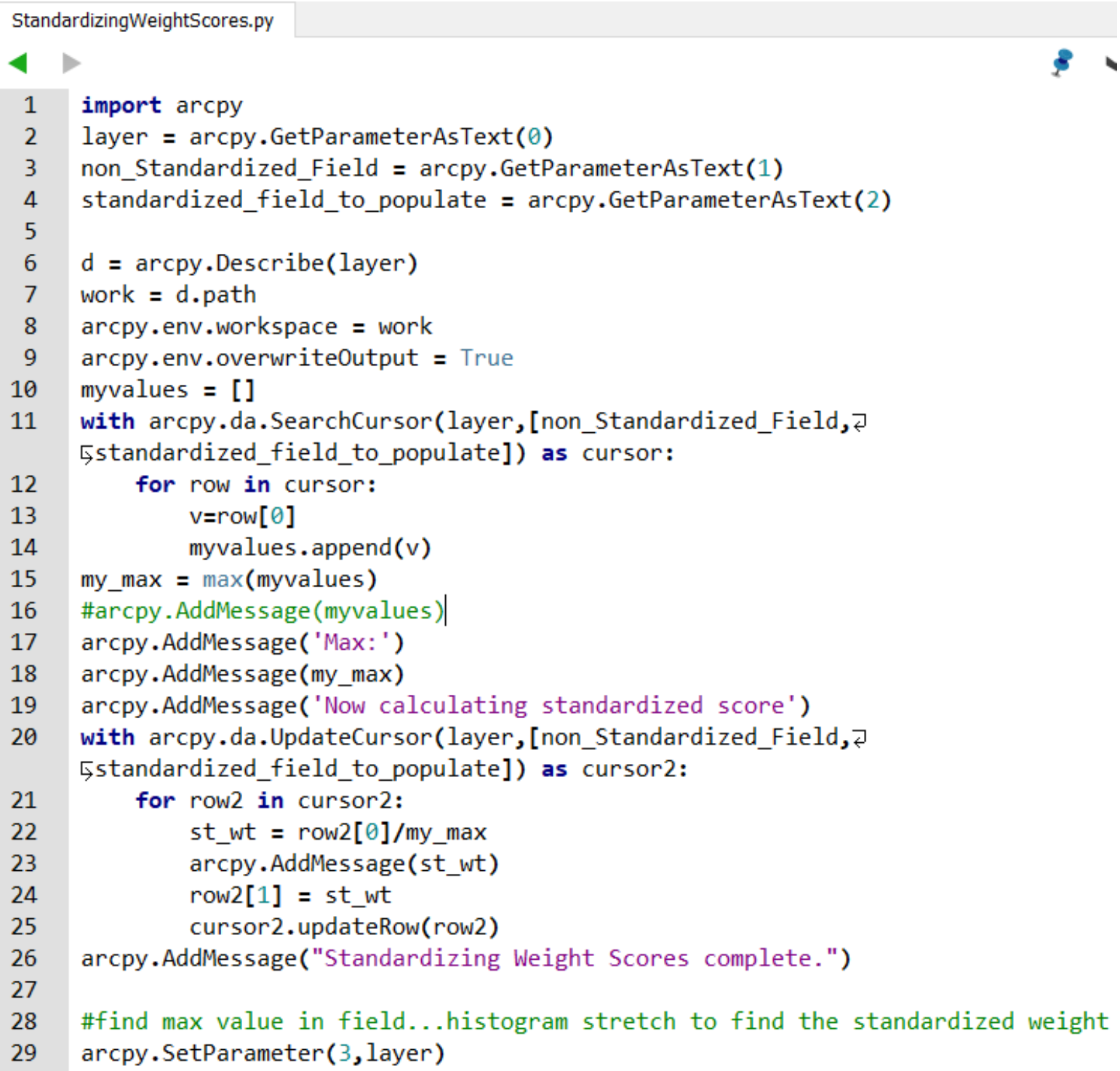

Figure A-19: StandardizingWeightScores.py

The StandardizingWeightScores.py script was integrated into a script tool such that parameter 0 and parameter 3 had the data type of "Feature Layer" and parameters 1 and 2 had the data type of "Field." Parameters 0, 1, and 2 were required inputs, with parameters 1 and 2 being dependent on parameter 0 . Parameter 3 was a derived output. 


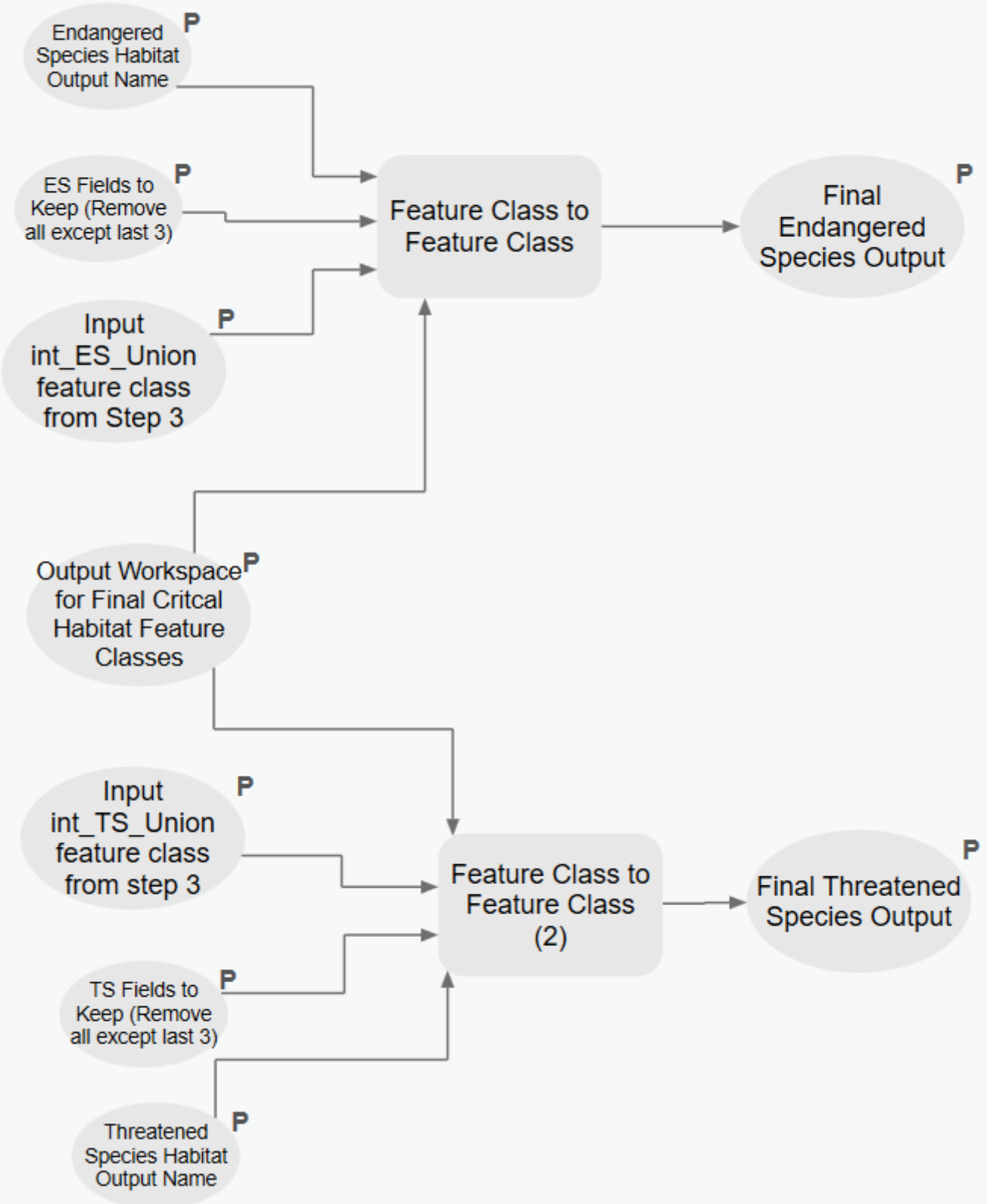

Figure A-20: CritHabPolys_Step4 


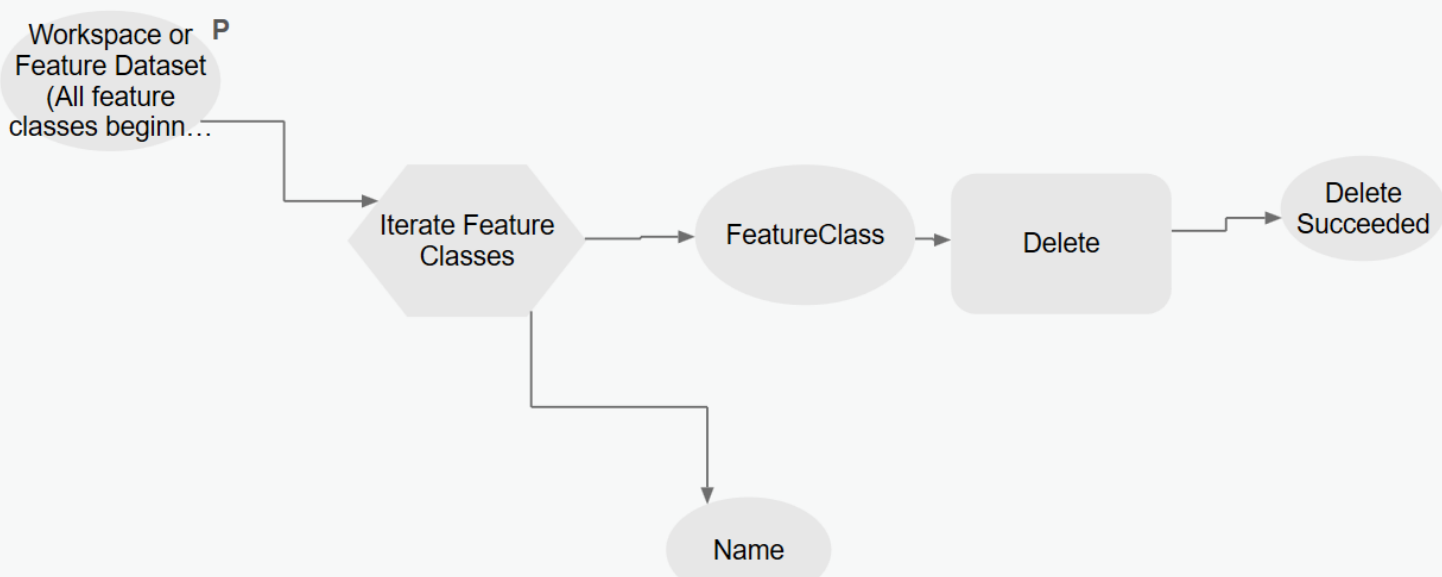

\section{Figure A-21: CritHabPolys_Step5_CleanUp}

Figures A-22 to A-24 show the user interface for the CritHabPolys model tools.

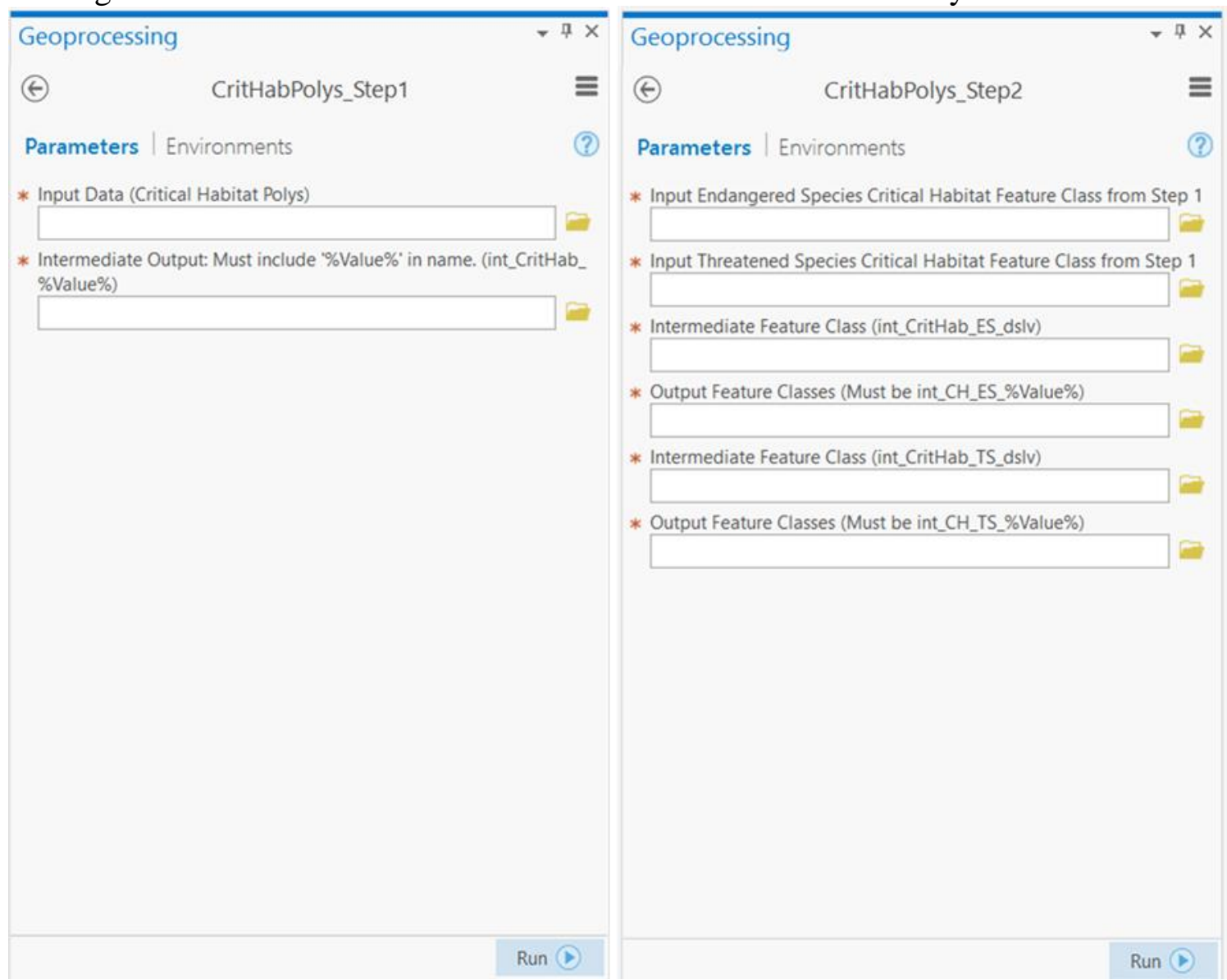

Figure A-22: User Interface for CritHabPolys_Step1 and CritHabPolys_Step2 


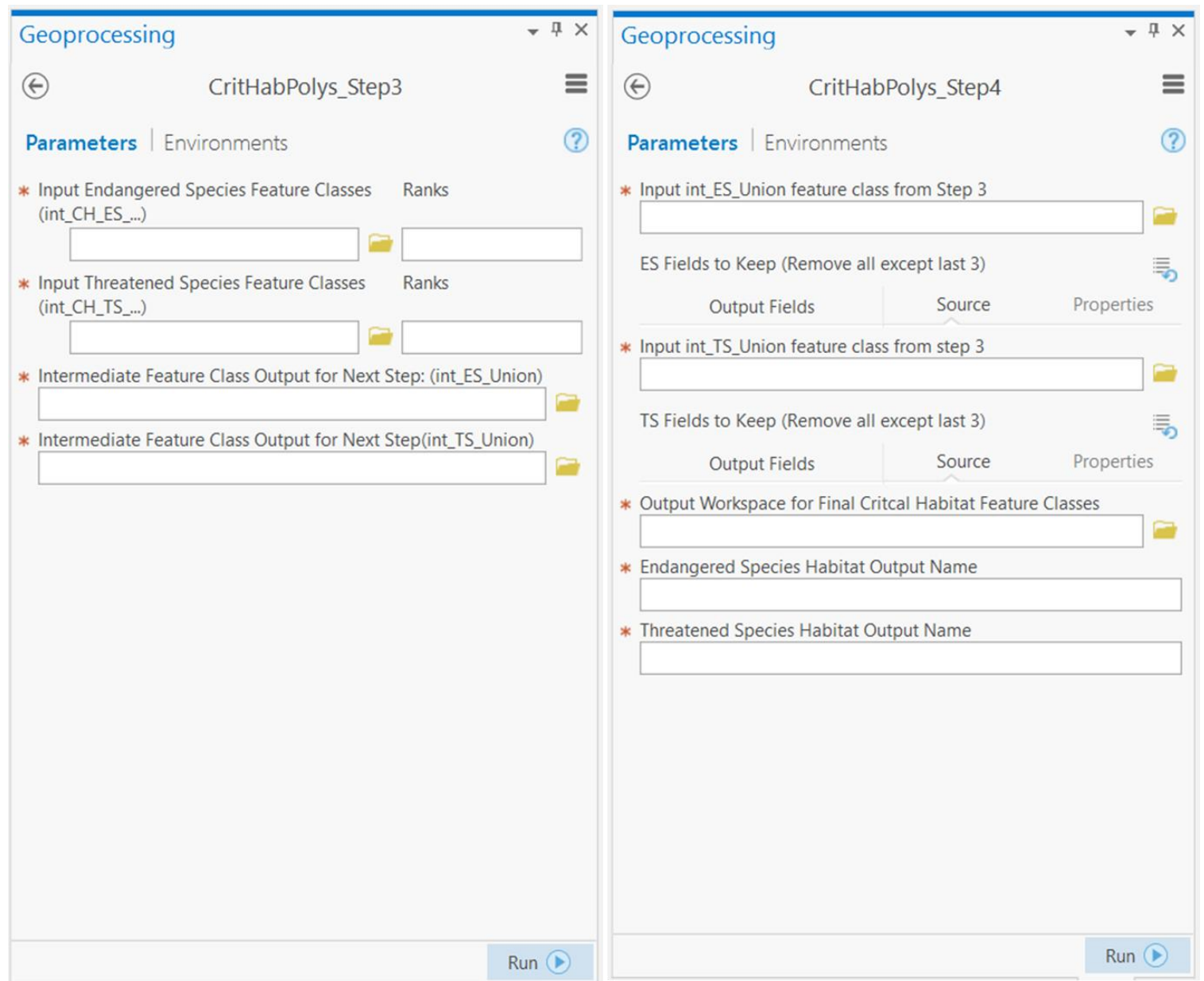

Figure A-23: User Interface for CritHabPolys_Step3 and CritHabPolys_Step4 


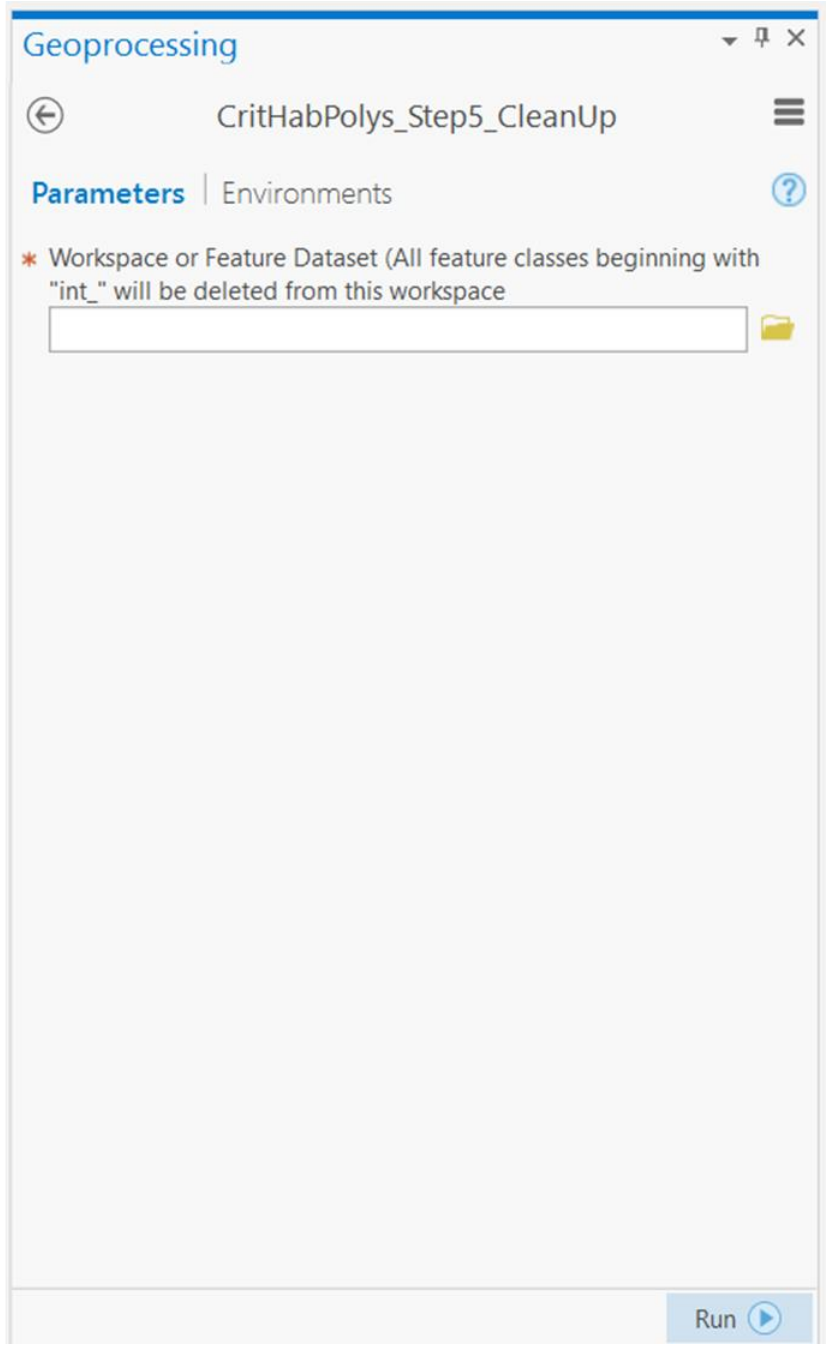

Figure A-24: User Interface for CritHabPolys_Step5_CleanUp

\section{Important Bird Areas Data Prep}

Important Bird Areas Data Prep was the final data preparation toolset and also the smallest, as the data preparation process for the Important Bird Areas data was simpler than the critical habitat data. Figure A-25 shows the two models in the toolset.

4 Ex Important Bird Areas Data Prep

$$
\begin{aligned}
& \text { ImportantBirdAreas Step1 } \\
& \text { ImportantBirdAreas Step2 (CleanUp) }
\end{aligned}
$$

Figure A-25: Important Bird Areas Data Prep Toolset 
Figures A-26 through A-27 show the two steps in the Important Bird Areas Data Prep toolset.

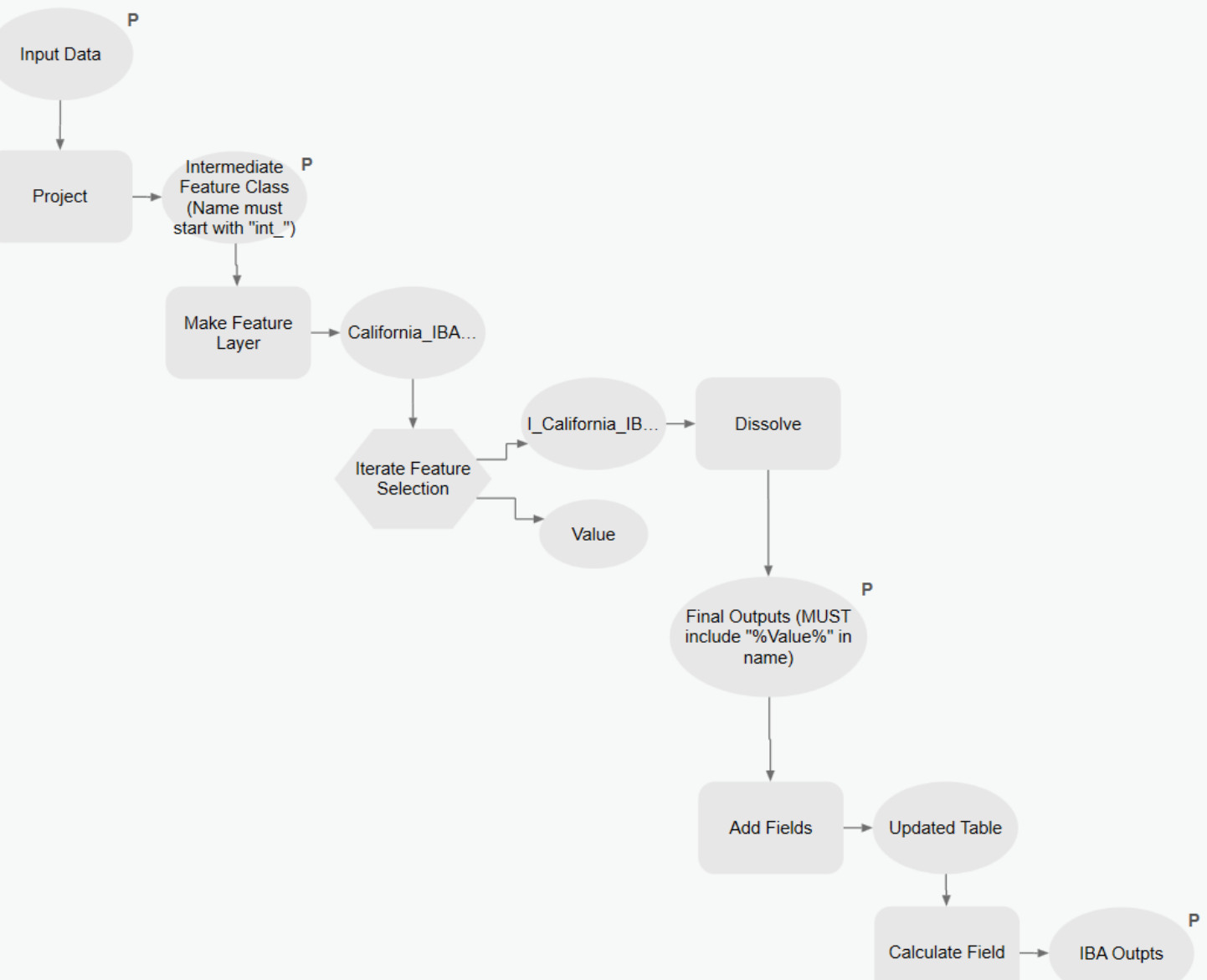

\section{Figure A-26: Important Bird Areas Data Prep Step 1}

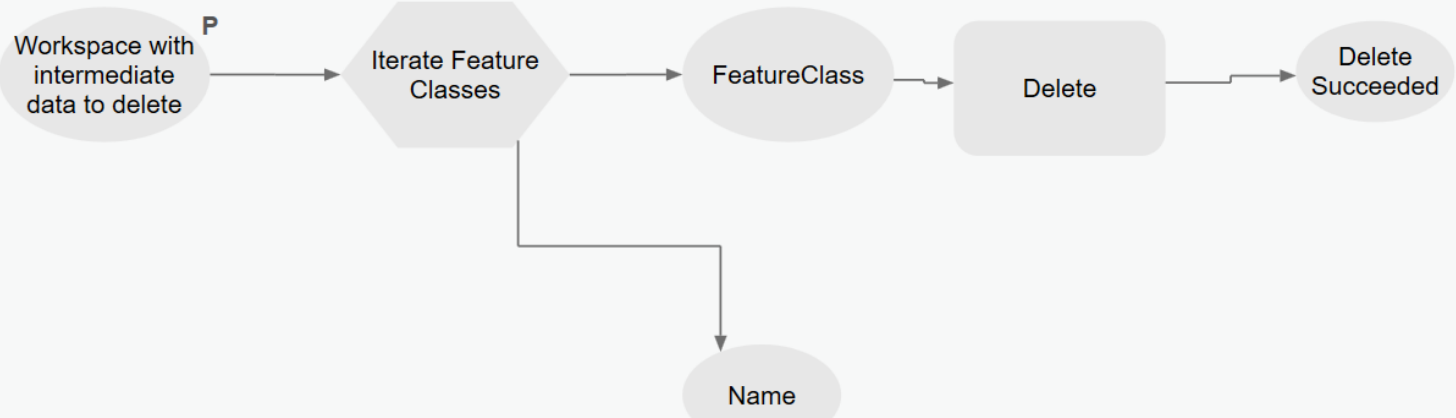

Figure A-27: Important Bird Areas Data Prep Step 2 
Figure A-28 shows the user interface for ImportantBirdAreas Step1 and Step 2.

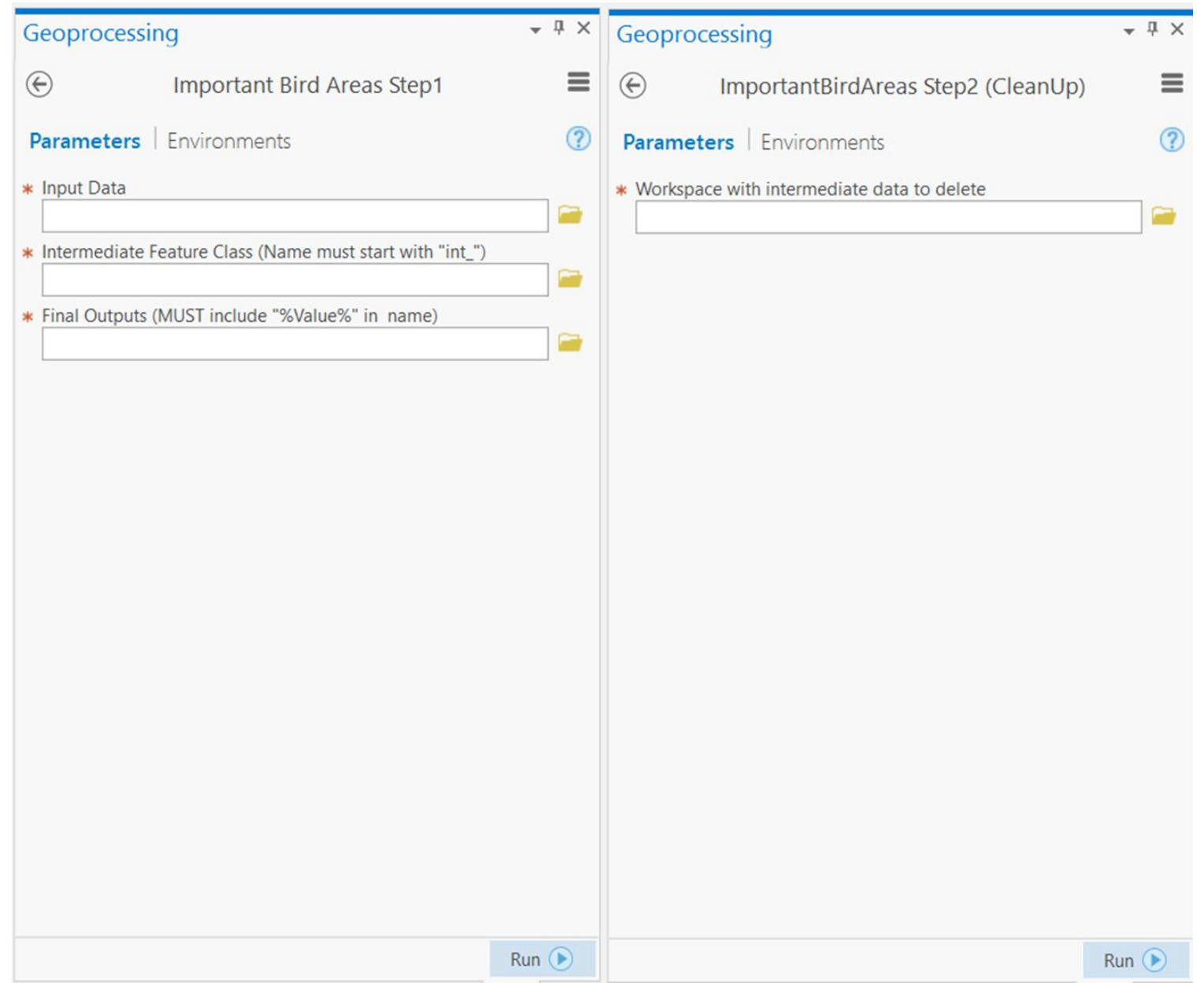

Figure A-28: User Interface for ImportantBirdAreas Step1 and ImportantBirdAreas Step 2 (Clean Up)

\section{Wetland Prioritization Analysis}

The final toolset in the analysis was the Wetland "Prioritization Analysis" and consisted of 4 steps and a background process (Figure A-29).

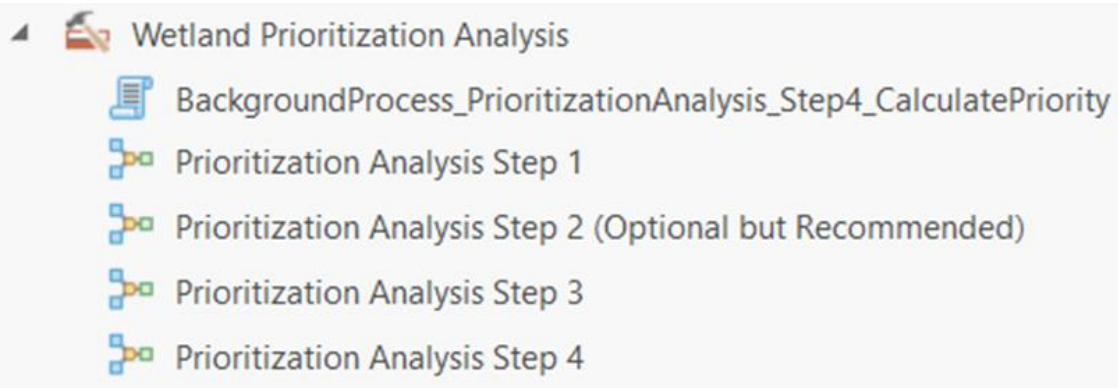

Figure A-29: Wetland Prioritization Analysis 
Figure A-30 to A-25 show the details of the models and script tools in the Wetland Prioritization Analysis toolset.

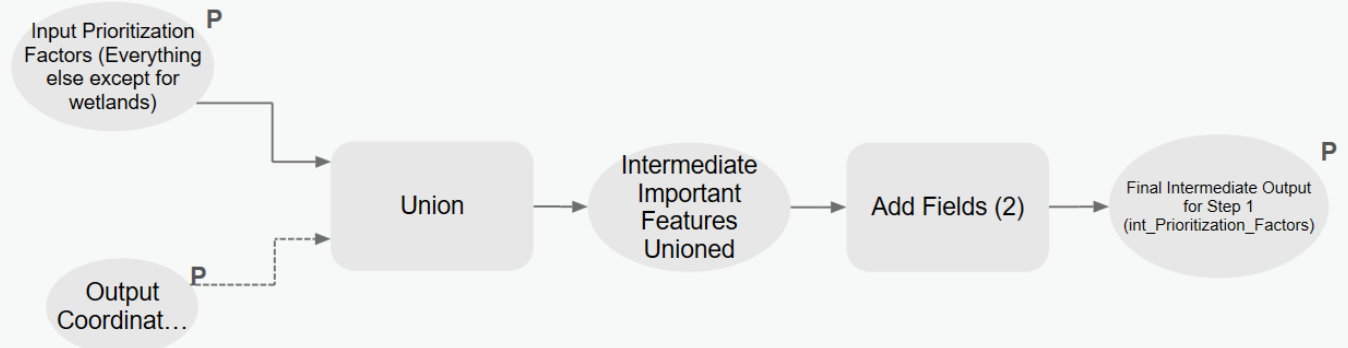

Figure A-30: Prioritization Analysis Step 1

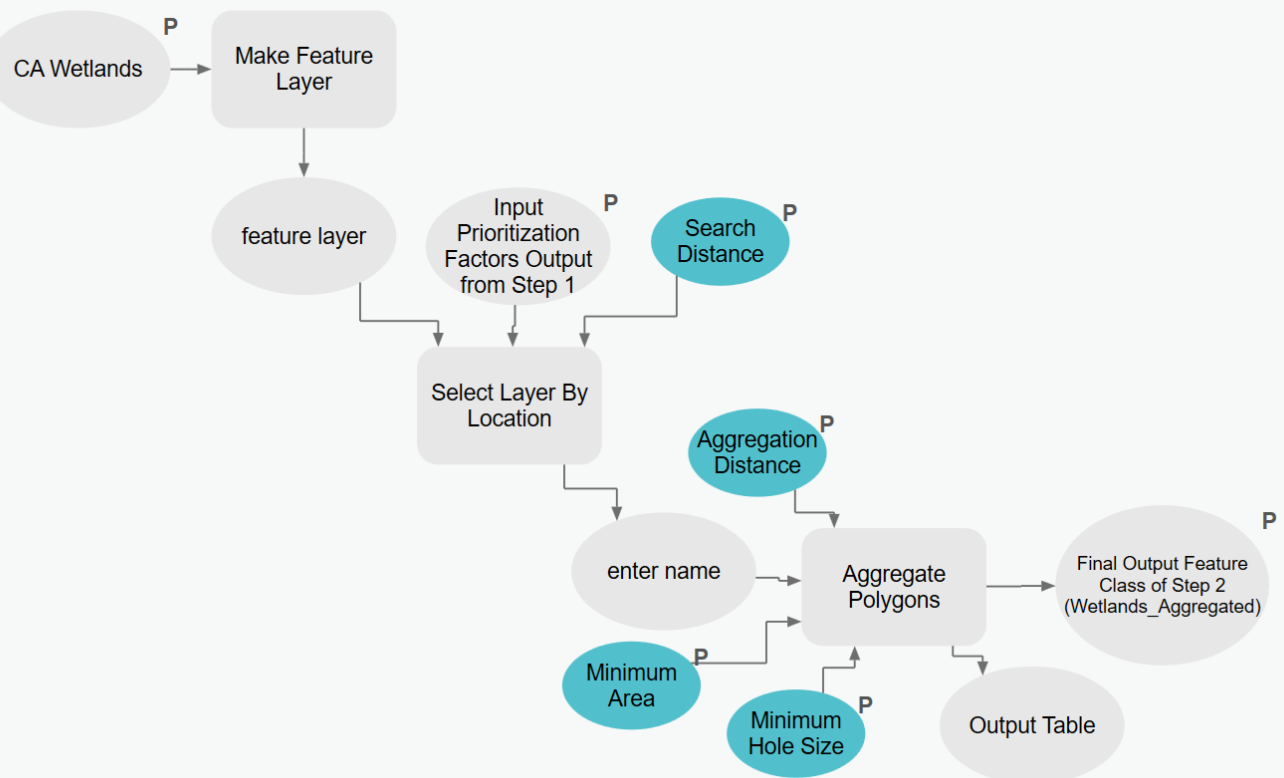

Figure A-31: Prioritization Analysis Step 2 (Optional but Recommended)

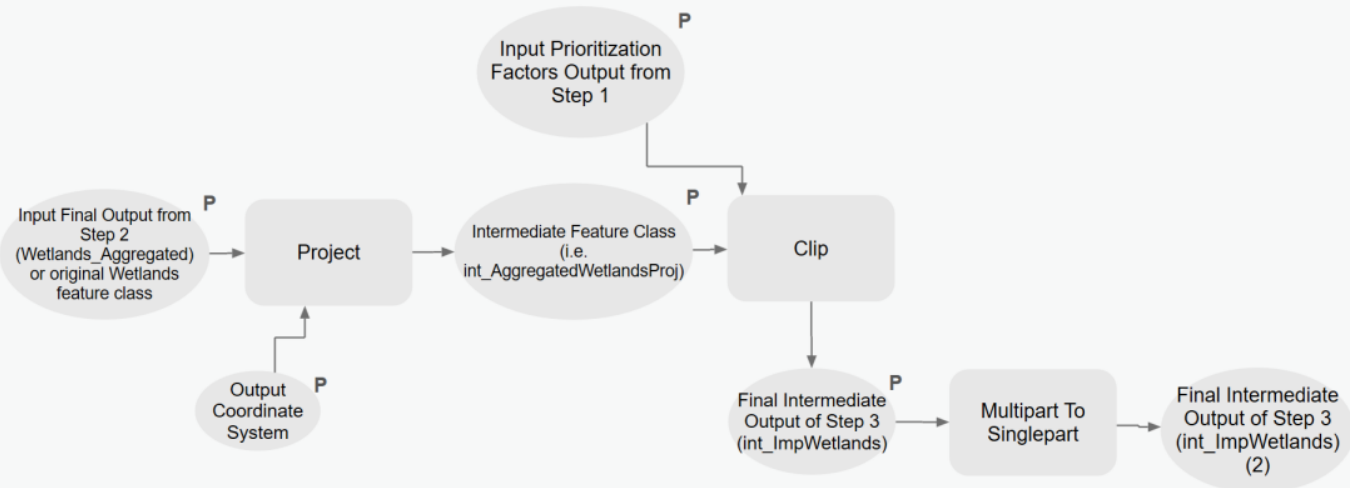

Figure A-32: Prioritization Analysis Step 3 


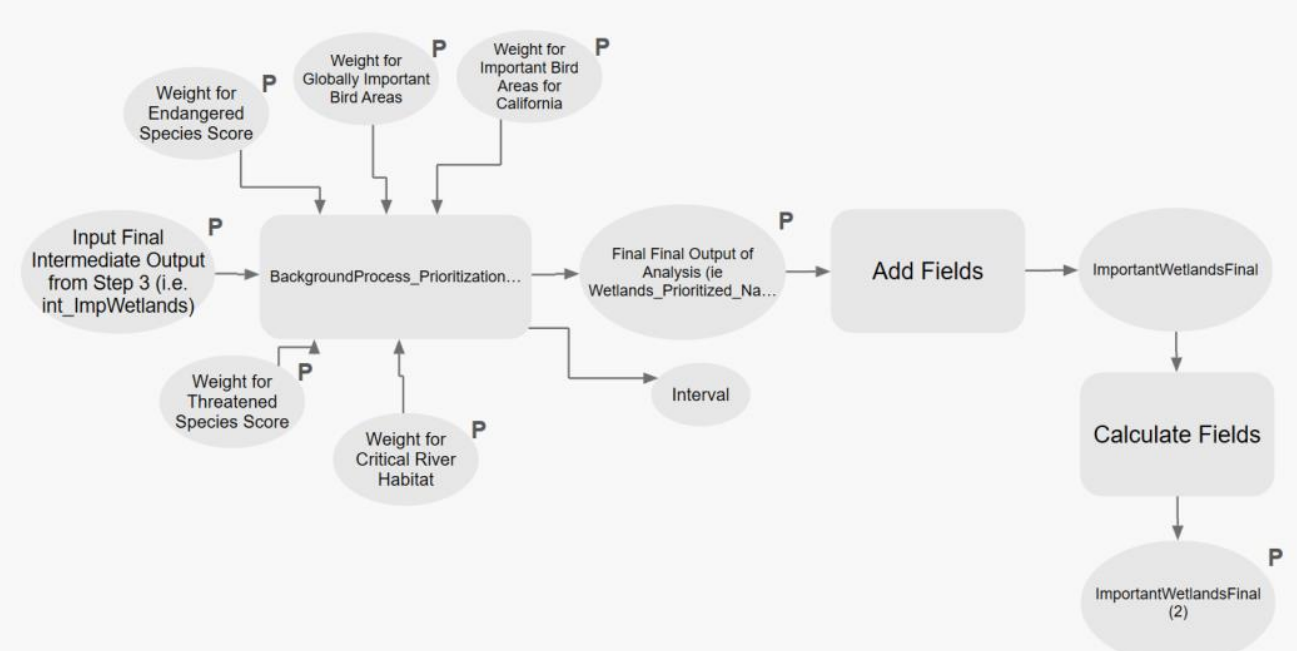

Figure A-33: Prioritization Analysis Step 4

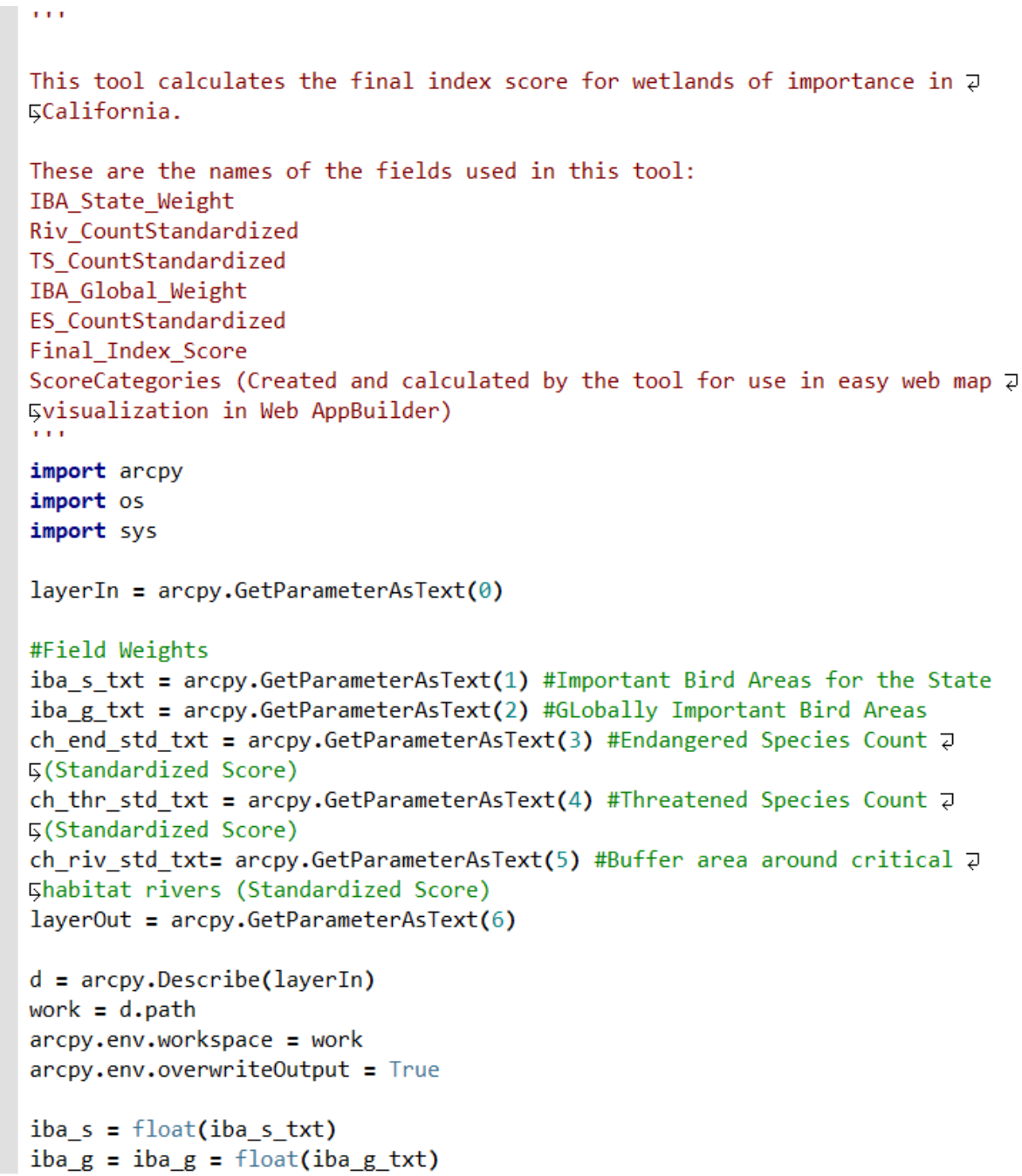

Figure A-34: BackgroundProcess_Prioritization Script Tool (CalculateFinalScore_Pro_Version2.py) rows 1-34 


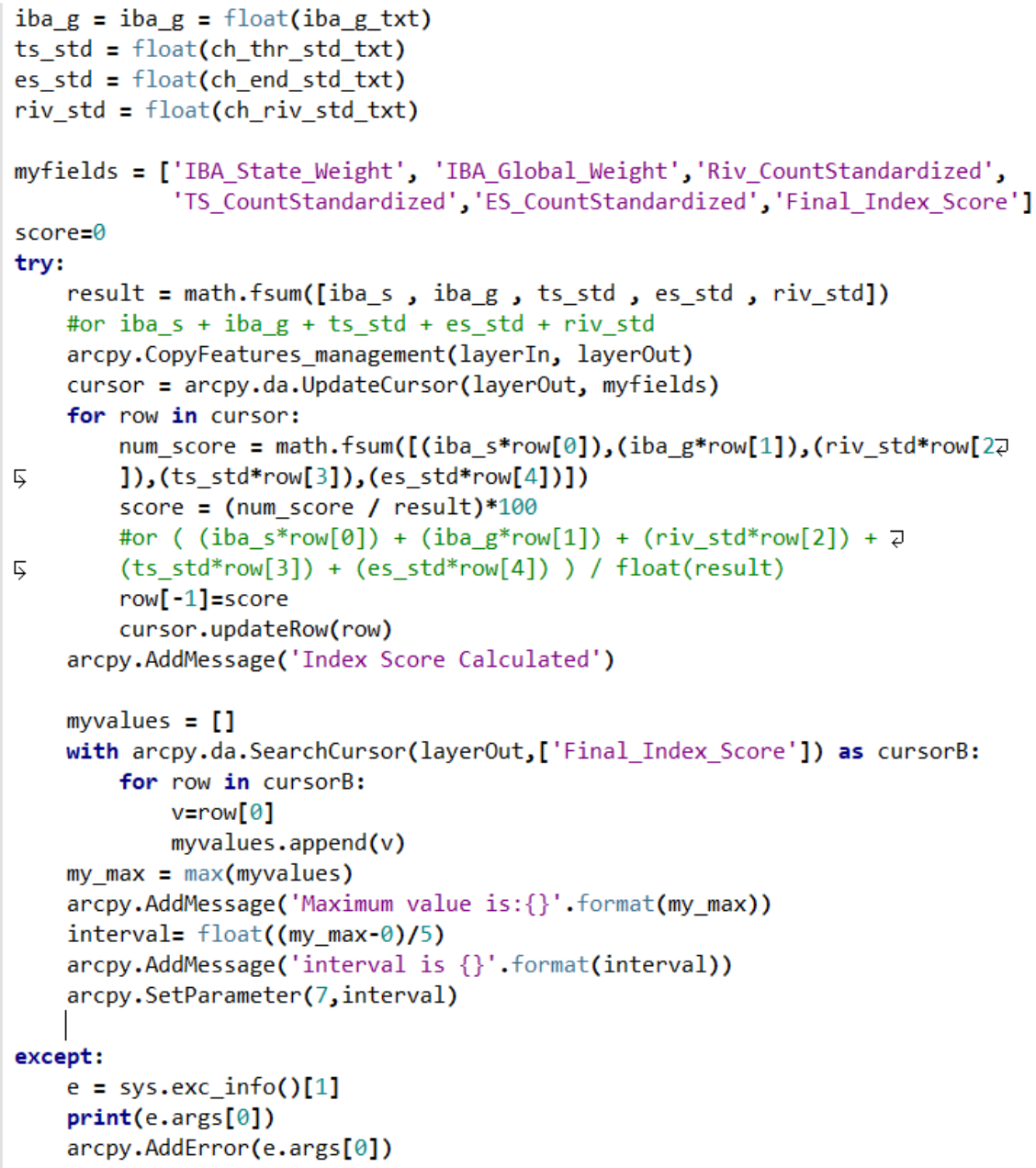

Figure A-35: BackgroundProcess_Prioritization Script Tool (CalculateFinalScore_Pro_Version2.py) rows 34-70

Figure A-39 shows the parameter settings used to integrate

CalculateFinalScore_Pro_Version2.py into a script tool.

Tool Properties: BackgroundProcess_PrioritizationAnalysis_Step4_CalculatePriority

\begin{tabular}{|c|c|c|c|c|c|c|c|c|}
\hline General & \multicolumn{8}{|c|}{ Define the script tool parameters } \\
\hline Parameters & & Label & Name & Data Type & Type & Direction & Category & Fil \\
\hline \multirow[t]{10}{*}{ Validation } & 0 & Input Layer & Input_Layer & Feature La... & Required & Input & & \\
\hline & 1 & Weight for... & Weight_for_State_IBAs & Double & Required & Input & & \\
\hline & 2 & Weight for... & Weight_for_Global_IBAs & Double & Required & Input & & \\
\hline & 3 & Weight for... & Weight_for_Count_of_Endangered_Species & Double & Required & Input & & \\
\hline & 4 & Weight for... & Weight_for_Count_of_Threatened_Species_(standardized) & Double & Required & Input & & \\
\hline & 5 & Weight for... & Weight_for_River_Habitat_(for_SteelheadTrout_and_Chinook_salmon) & Double & Required & Input & & \\
\hline & 6 & Output La... & Output_Layer & Feature $\mathrm{Cl} \ldots$ & Required & Output & & \\
\hline & 7 & Interval & Interval & Double & Derived & Output & & \\
\hline & * & & & String & Required & Input & & \\
\hline & \multicolumn{8}{|c|}{4} \\
\hline & & & & & & & & \\
\hline
\end{tabular}

Figure A-39: Parameters used to Integrate CalculateFinalScore_Pro_Version2.py into a Script Tool 


\section{CalculateFinalScore_Web_Version2}

For the geoprocessing service that was created, a script tool was created and then imbedded inside a model in an ArcMap 10.5 toolbox (Figure A-40)

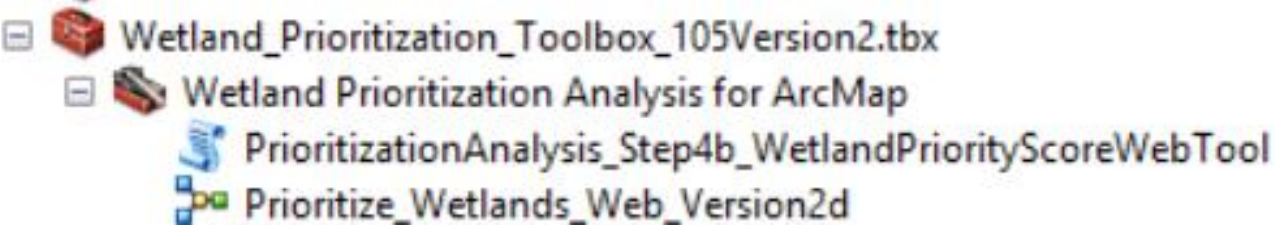

Figure A-40: Wetland Prioritization Toolbox for ArcMap 10.5

Figures A-41 to A-44 show the model and script tool contained in the toolbox.

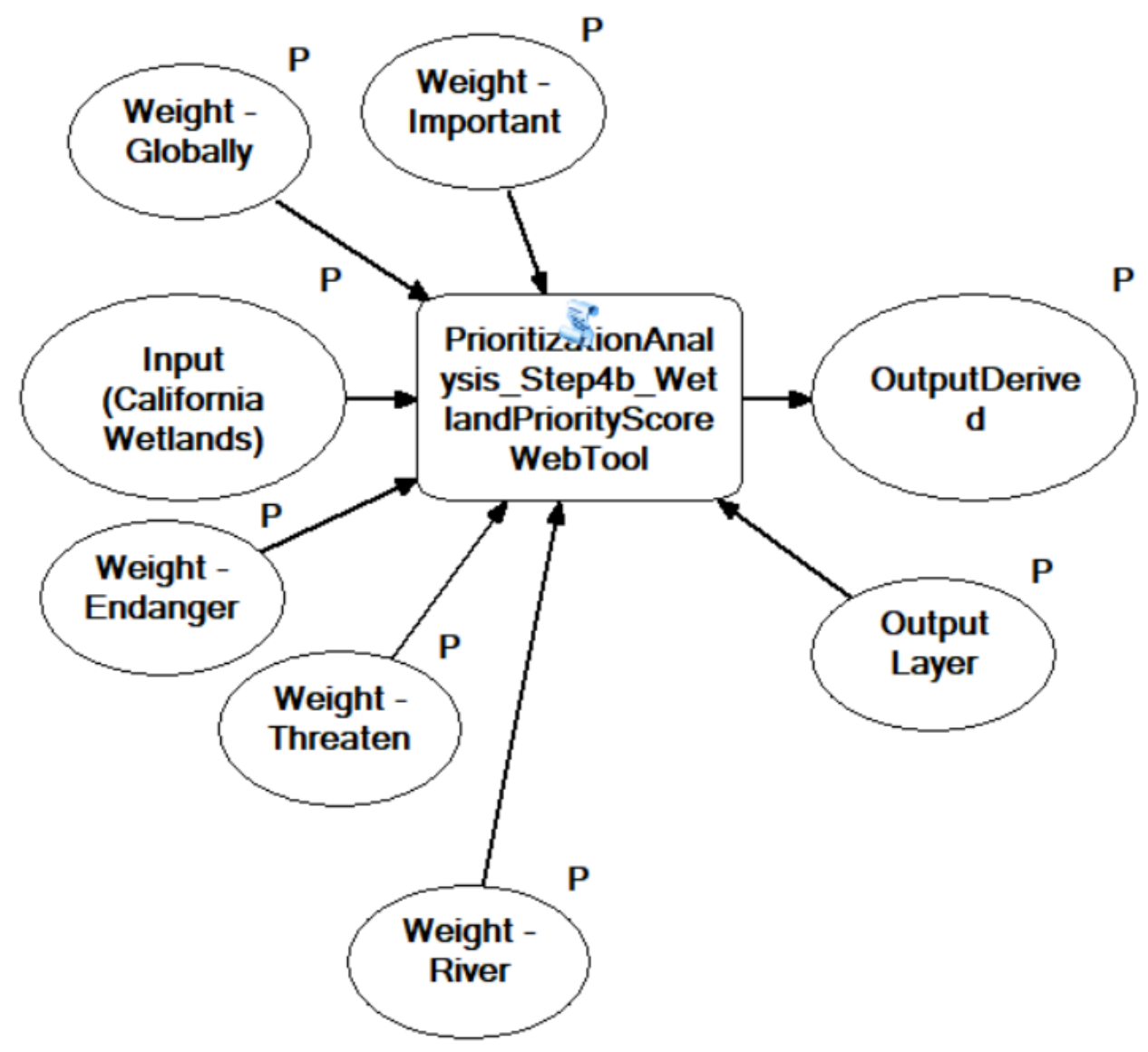

Figure A-41: PrioritzeWetlands_Web_Version2d 


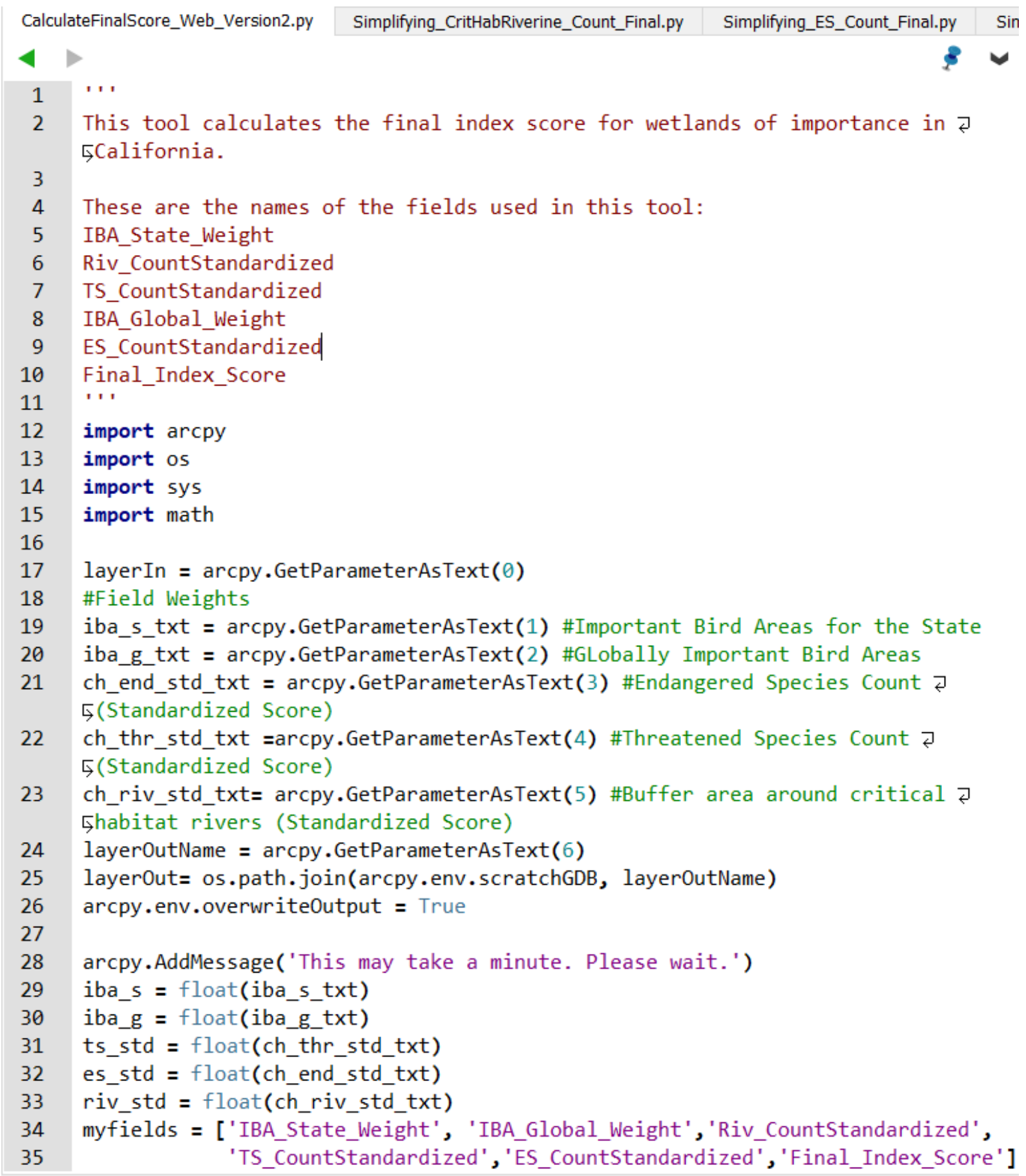

Figure A-42: CalculateFinalScore_Web_Version2.py Rows 1-35 


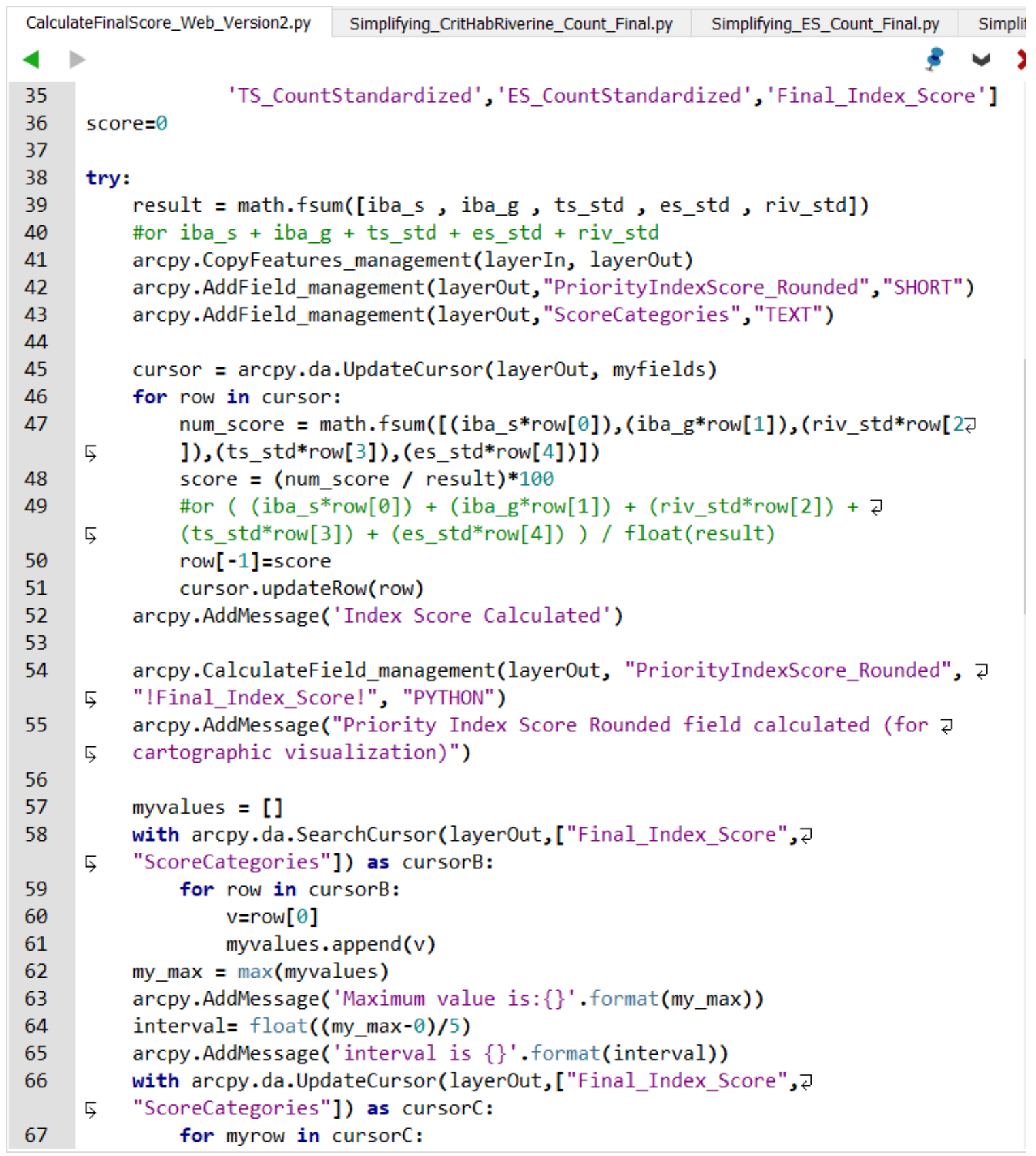

Figure A-43: CalculateFinalScore_Web_Version2.py Rows 35-67 


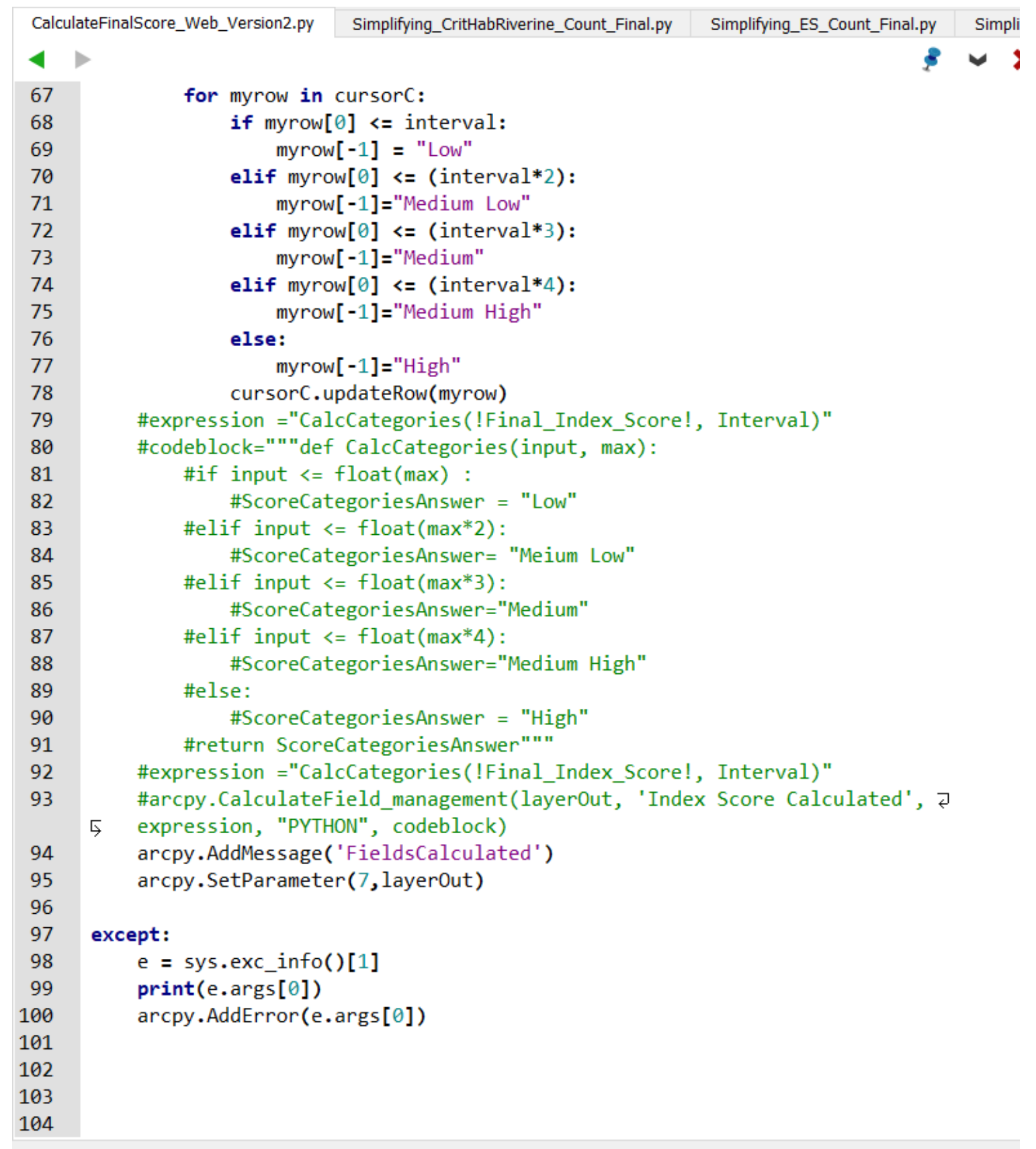

Figure A-44: CalculateFinalScore_Web_Version2.py Rows 67-100

Figure A-45 shows the parameter settings used to integrate CalculateFinalScore_Web_Version2.py into a script tool. Most notably, the input was set as a "Feature Set" and the output as a "Feature Class." In addition, OutputDerived was given the parameter as being derived from the input wetlands layer, and symbology for the output was set according to a pre-defined symbology layer file that was created in ArcMap. 


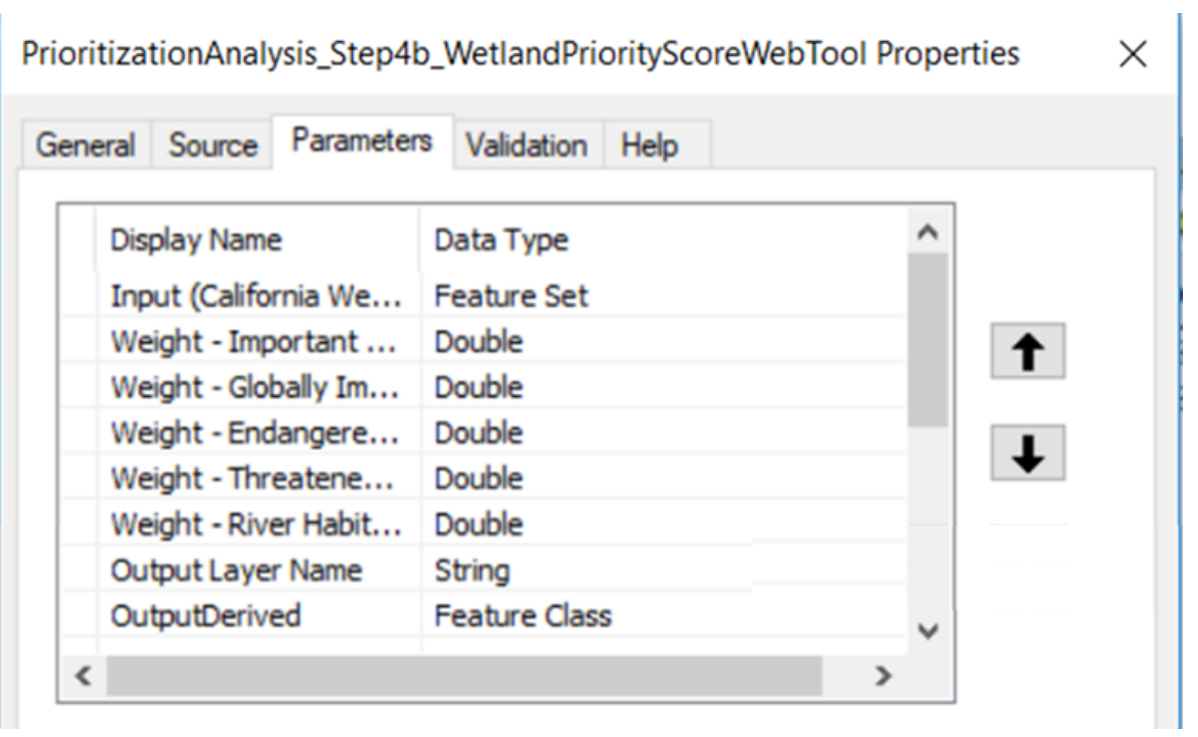

Figure A-45: Script tool parameters in PrioritizationAnalysis_Step4b_WetlandPriorityScoreWebTool

\section{Publishing the Geoprocessing Service}

The process that was used to publish the geoprocessing service from ArcMap is very standard, so most of the settings will not be specified here, except that the maximum number of records returned by the server should be increased to an appropriate number depending on the size of the input dataset (the maximum number should not be less than the number of features in the feature class). In this project, that number was specified as 3000. The service was also published as asynchronous. It should also be noted that when republishing the geoprocessing service, the input mode should be specified as "constant value" (See Figure A-46).

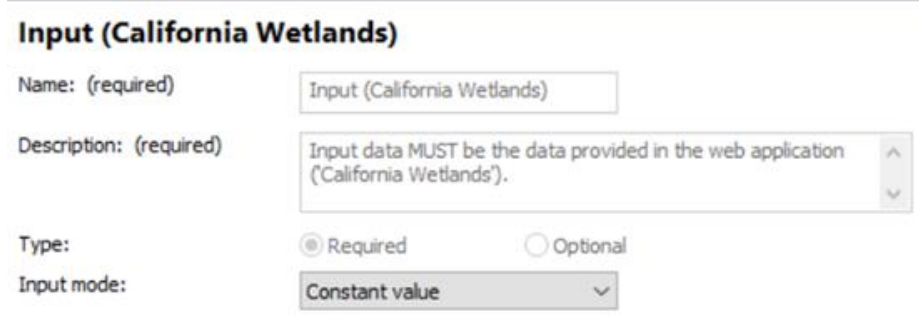

Figure A-46: When publishing the service, input should be "Constant value"

In addition, the Output_Layer_Name parameter in the model/script tool was used for convenience in running the tool in a desktop program but is unnecessary for the geoprocessing service and confusing for users if integrated into the final web application. As such, the geoprocessing service should be configured in Web AppBuilder such that the Output_Layer_Name parmeter is not visible, as shown in Figure A-47. 


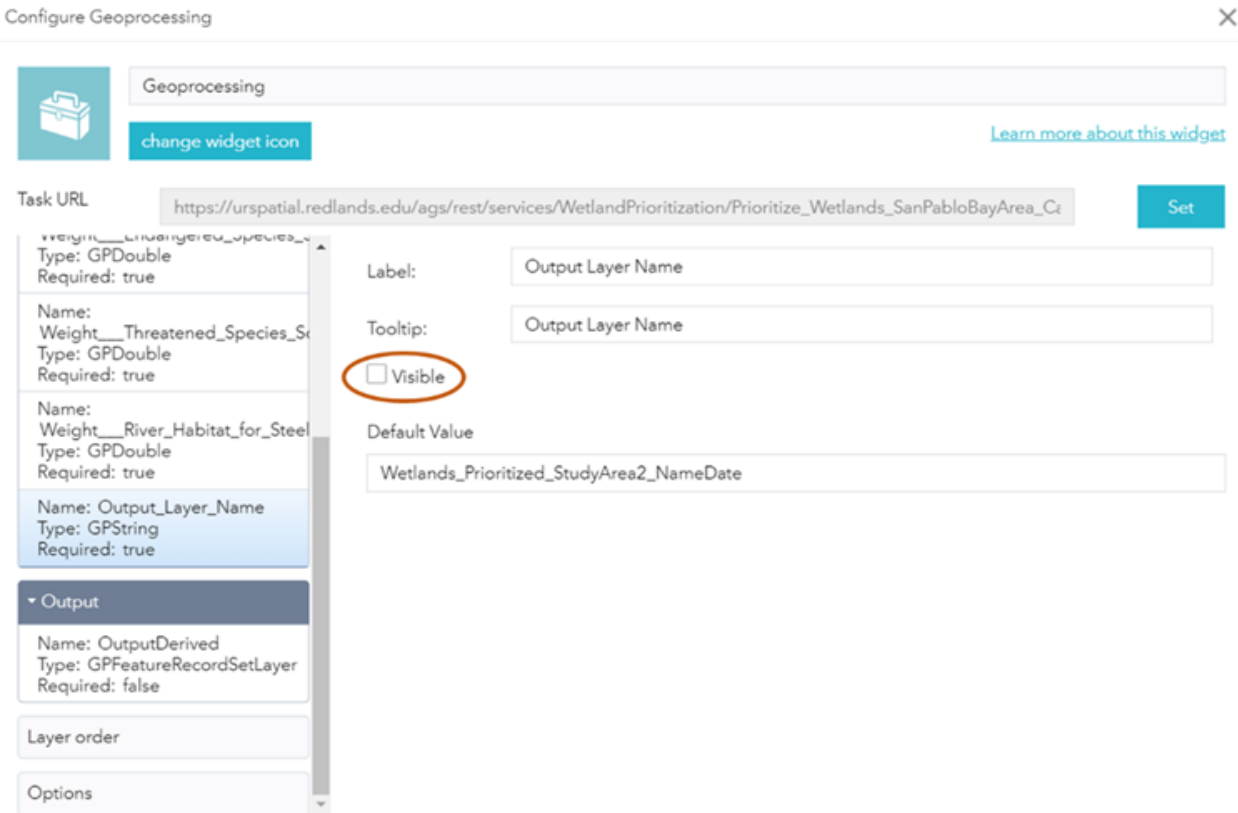

Figure A-47: Output_Layer_Name parameter should not be visible, when configuring the geoprocessing tool 